\title{
Many species of the Carnivora consume grass and other fibrous plant tissues
}

\author{
Alan R. Franck ${ }^{1, *}$ \& Arian Farid ${ }^{2}$ \\ ${ }^{1}$ Department of Biological Sciences, Florida International University, Miami, Florida, U.S.A. \\ ${ }^{2}$ Herbarium, Department of Cell, Molecular, and Microbiology, \\ University of South Florida, Tampa, Florida, U.S.A. \\ ${ }^{*}$ Corresponding author: afranck@fiu.edu
}

\begin{abstract}
Within the Carnivora order, the consumption of fibrous plant tissues (FPT), such as leaves and stems, is only known to serve the nutritional needs of eight species in the Ailuridae and Ursidae. Apart from the Ailuridae and Ursidae, the extent of FPT ingestion in the Carnivora is poorly understood. A literature search was conducted to compile studies containing evidence of FPT consumption in the Carnivora, primarily based on analyses of scats or gastrointestinal tracts. Among 352 studies, there was evidence of FPT consumption in any amount in 124 species, or $41 \%$, of the Carnivora. Grass consumption was documented in 95 species, while ingestion of sedges, marine plants, bryophytes, conifers, and dicots was much less frequent. A few species showed evidence of consuming fungi or soil. Nine studies observed co-occurrences of intestinal parasites with grasses or sedges in the scats of the Carnivora, suggesting these abrasive or hairy plant tissues help to expel intestinal parasites. The relevance of consuming marine plants, bryophytes, conifers, dicots, fungi, or soil has also been underappreciated. Deliberate ingestion of FPT may be more widespread and important than previously realized in the Carnivora.
\end{abstract}

Keywords. Algivory, carnivore, folivory, fungivory, geophagy, herbivory.

FrancK A.R. \& FARID A. (2020). Many species of the Carnivora consume grass and other fibrous plant tissues. Belgian Journal of Zoology 150: 1-70. https://doi.org/10.26496/bjz.2020.73

\section{Introduction}

The Carnivora order contains 300 extant species among 129 genera and 16 families (AGNARsson et al. 2010; NyaKaTURA \& Bininda-Emonds 2012; JACKSON et al. 2017; Zhou et al. 2017; Burgin et al. 2018). Their relatively simplistic gastrointestinal tract (MCGrosky et al. 2016) is well adapted to carnivory, and approximately $98 \%$ of the species are carnivorous, frequently consuming invertebrate or vertebrate animals (see PINEDA-MunOz \& Alroy 2014). About $58 \%$ of the order are considered predominantly carnivorous, feeding primarily on animals, and about $40 \%$ are predominantly omnivorous, with significant portions of the diet coming from both animals and plants (NowAK 2005; KIssLING et al. 2014; GAINSBURY et al. 2018). Only six species were classified as predominantly herbivorous: Ailuropoda melanoleuca, Ailurus fulgens, Ailurus styani, Paradoxurus jerdoni, Tremarctos ornatus, and Ursus thibetanus (BURGIN et al. 2018; GAINSBURY et al. 2018). 
Fruits are one of the most important plant dietary items, consumed by nearly all omnivorous and herbivorous Carnivora species (QuAdros \& Monteiro-Filho 2000; Gainsbury et al. 2018), being especially important in the Ursidae and Viverridae (Corlett 2017), with one species, Paradoxurus jerdoni, considered predominantly frugivorous (GAINSBURY et al. 2018). Frugivory is generally absent in the Eupleridae, Felidae, Odobenidae, Otariidae, Phocidae, and Prionodontidae (GaInsBury et al. 2018), although there are occasional reports of fruit consumption in the Eupleridae (DURBIN et al. 2010) and the Felidae (Schaller 1967; Turkowski 1980; SMythe 1986; Romo 1995; TABer et al. 1997; Rosalino \& SAntos-Reis 2009; CoRlett 2017).

Consumption of seeds, roots, or nectar is relatively infrequent overall in the Carnivora. Granivory occurs in some species of the Ailuridae, Canidae, Herpestidae, Mephitidae, Mustelidae, Procyonidae, Ursidae, and Viverridae (Amaral 2007; Nadeem et al. 2012; CZernik et al. 2016; Gainsbury et al. 2018). Rhizovory occurs in some species of the Ailuridae, Herpestidae, Mustelidae, and Ursidae (KALLE et al. 2012; GAINSBURY et al. 2018). Nectarivory has been observed in Galerella pulverulenta (Herpestidae), Martes flavigula and M. gwatkinsii (Mustelidae), Potos flavus (Procyonidae), and Genetta tigrina (Viverridae) (Hutton 1944; Lack 1977; Kays 1999; Parr \& Duckworth 2007; Steenhuisen et al. 2015).

Herbivory of fibrous plant tissues (FPT; e.g., leaves and stems) is generally considered to serve the nutrional needs of only eight species in two families (Ailuridae and Ursidae) of the Carnivora: Ailuropoda melanoleuca (Schaller et al. 1989; Sims et al. 2007), Ailurus fulgens, A. styani (WeI et al. 2000; Panthi et al. 2012; Sharma et al. 2014), Tremarctos ornatus (Troya et al. 2004; GarcíARangel 2012), Ursus americanus (McLellan 2011), Ursus arctos (MCLellan 2011), Ursus maritimus (STEMPNiEWicz 2017), and Ursus thibetanus (Christiansen 2008; FurusaKa et al. 2017). Apart from these eight species, it is unknown how many other species of the Carnivora ingest FPT and what purpose it serves, although grasses, in particular, may serve to expel intestinal parasites (HuFFMAN \& CATON 2001; HART \& HART 2018).

We hypothesize that FPT consumption is widespread in the Carnivora, especially considering that domesticated species (i.e., cats and dogs) commonly consume FPT such as grass (HART 2011), suggesting it could be an innate behaviour (BJONE et al. 2009). Since herbivory of FPT is already well characterized in eight species (listed above), our main objective was searching for evidence of FPT ingestion in the other 292 extant species of the Carnivora. The types of FPT included were algae, bark, flowers, leaves, stems, and wood. Data for fungi consumption, an organismal group normally overlooked in the Carnivora (GAINSBURY et al. 2018), were also collated. The peculiar ingestion of soil evidenced in some studies was additionally noted.

\section{Material and methods}

The taxonomy here follows Burgin et al. (2018), with the exception of the recognition of Canis familiaris (JACKSON et al. 2017). Recently extinct species are not included in the calculations, i.e., Cryptoprocta spelea, Dusicyon australis, Dusicyon avus, Neomonachus tropicalis, Neovison macrodon, and Zalophus japonicus.

Our search strategy was to query each species, genus, family, or order, in combination with the terms "grass", "leaves", "plants", "stems", "vegetation", "vegetative", "fungi”, "soil”, "scat", or "stomach" in Google Scholar. References cited within studies were also screened. Our focus was on all species of the Carnivora, especially the 292 species not generally known to consume FPT (dietary reviews were provided for the eight species already well known to consume FPT: Ailuropoda melanoleuca, Ailurus 
fulgens, A. styans, Tremarctos ornatus, Ursus americanus, U. arctos, U. maritimus, and U. thibetanus). Studies were included if they provided evidence of the consumption of algae, bark, flowers, fungi, leaves, soil, stems, or wood. Rarely, studies reported searching for evidence of plant ingestion but found none; these studies were not included here.

For each study, the information compiled included the species of Carnivora, category of plant or fungal tissue ingested, quantitative data on ingestion frequency, and any pertinent notes related to plant, fungi, or soil ingestion. Some studies reported or implied that evidence of FPT herbivory was observed, but explicitly excluded it from the data collection; hence, in these studies quantitative data are not available.

The studies included used a diverse array of terminologies, which were standardized as much as possible to present them as a common category here. The category algae used here includes the terms algae, kelp, and seaweed used in the studies. The category fungi used here includes the terms fungi and mushrooms used in the studies. The category fruit used here includes the terms berries and fruits used in the studies. The category grass here comprises the terms Graminae, graminoids, grass, and Poaceae. Leaves includes the terms leaf or leaves. Moss includes Bryophyta, bryophytes, and moss. Needles includes the terms conifer needles and needles. The category plant includes the terms herbaceous plants, herbs, forbs, plant(s), plant content, plant food, plant fragments, (unidentified) plant material, plant matter, plant remains, plant remnants, and Plantae. The category root includes the terms roots and tubers. Sedge includes the terms Cyperaceae, Cyperales, and sedge. The term Poales in a study was interpreted as including both the grass and sedge categories. Soil includes the terms dirt, sand, and soil. The category stem includes the terms branches, stems, sticks, twigs, and woody material. The category vegetation includes the terms bracts, casuarina needles (presumably actually referring to its stem and whorls of leaves), fibers or fibres, undigested leaves, scales, undigestible plant material, vegetable material, vegetable matter, vegetation, and vegetative. Other categories used here were equivalent to a single term found in the study, such as bark, digested grass, flowers, hair, lichens, miscellaneous, molluscs, plastic, seeds, shells, trap-pan covers, wood, and Zosteraceae. Although categories such as fruit, hair, miscellaneous, plastic, roots, seeds, shells, and trap-pan covers were not the focus of this study, they are included here when they were grouped with other forms of plant eating and not reported individually.

The frequency of occurrence (FO), the most commonly used statistic (KLARE et al. 2011a), was the primary quantitative datum compiled from the studies. The FO here is the presence/absence of a plant or fungal category in each sample (usually a scat or stomach) given as a percent of the total number of samples. The FO was sometimes reported as the itemized frequency, being the number of individual food items of one category relative to the total number of food items found.

Sometimes the FO or data to calculate the FO were not provided. In these instances, other data were given such as the mass, relative frequency (RF), relative mass (RM), or relative volume (RV). The mass is the dried mass of a given item. The RF is the FO of one category divided by the sum of all the FOs, which standardizes the FOs so that the sum of all RFs totals $100 \%$. The RM is the mass of one category divided by the sum of all masses, so that the sum of all RMs totals $100 \%$. The RV is the volume of one category divided by the sum of volumes, so that the sum of all RVs totals $100 \%$. Direct observations of animals feeding on FPT were sometimes provided.

Percentages were rounded to the nearest whole number, except anything less than $1 \%$ was reported as $<1 \%$. Some studies reported data from different times or locations, but did not summarize the data. In these instances, data were summarized for each species within the particular study.

Personal observations were made of a mixed-breed dog (Canis familiaris) in Florida, USA from the ages of 4-10 during leashed walks or when the dog was roaming freely. Additional observations were made 
of two adult terriers (C. familiaris) in their yard in Seattle, Washington, USA (Appendix 1). These dogs were all privately owned and were only observed during their normal daily routines. No experimentation was conducted; no manipulation of any sort was enacted. No permissions or licences were necessary.

\section{Results}

The number of published studies included was 357 (Table 1), with some studies including multiple species. Five studies reported only the consumption of fungi but not FPT (DeLIBES 1978; Grenfell \& FASENFEST 1979; Zielinski et al. 1999; Helldin 2000; MatTsOn et al. 2002). From 352 studies, there were a total of 124 species and one hybrid from 72 genera and 12 families of Carnivora that showed evidence of consuming FPT (Fig. 1). Eight references were provided as dietary reviews of the eight species already well known to consume FPT, the species in the Ailuridae (Ailurus fulgens and A. styani) and Ursidae (Ailuropoda melanoleuca, Tremarctos ornatus, Ursus americanus, U. arctos, U. maritimus, and $U$. thibetanus). The remaining 344 studies documented FPT consumption in 116 species not generally considered to be folivores or algivores for nutritional needs. For seven species among seven genera

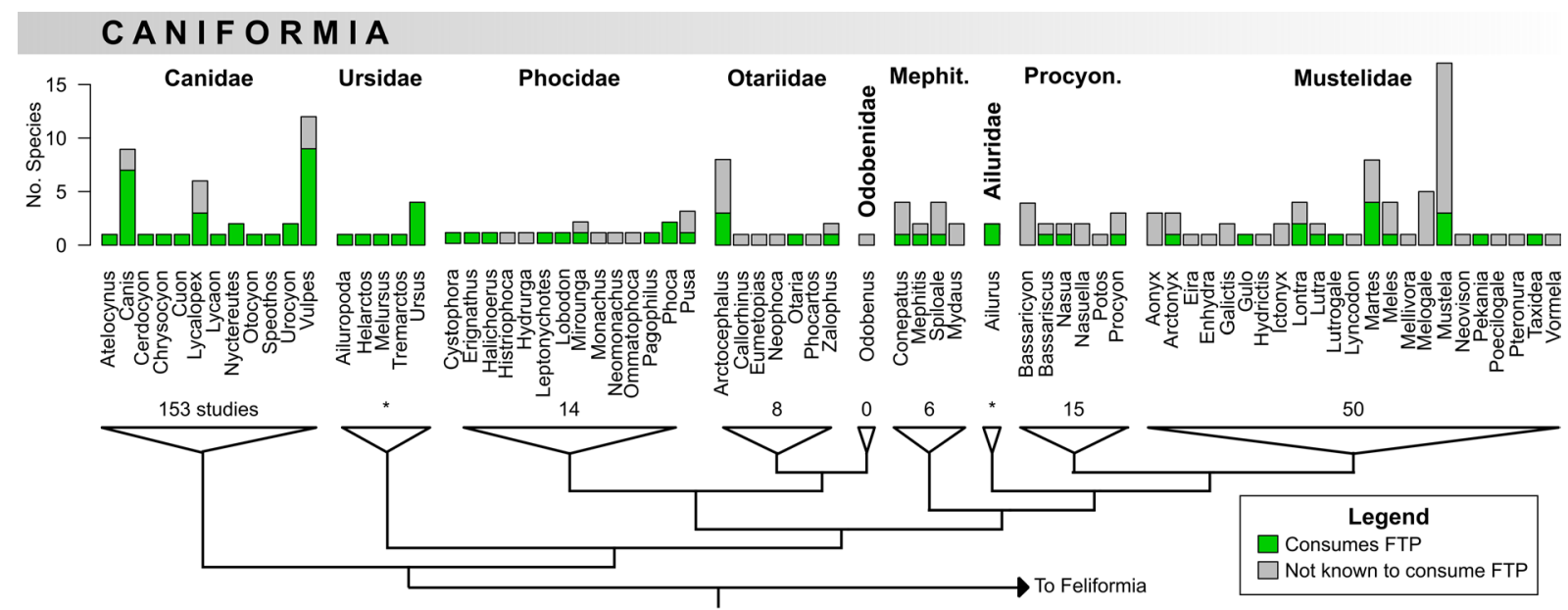

FE LIF OR M I A

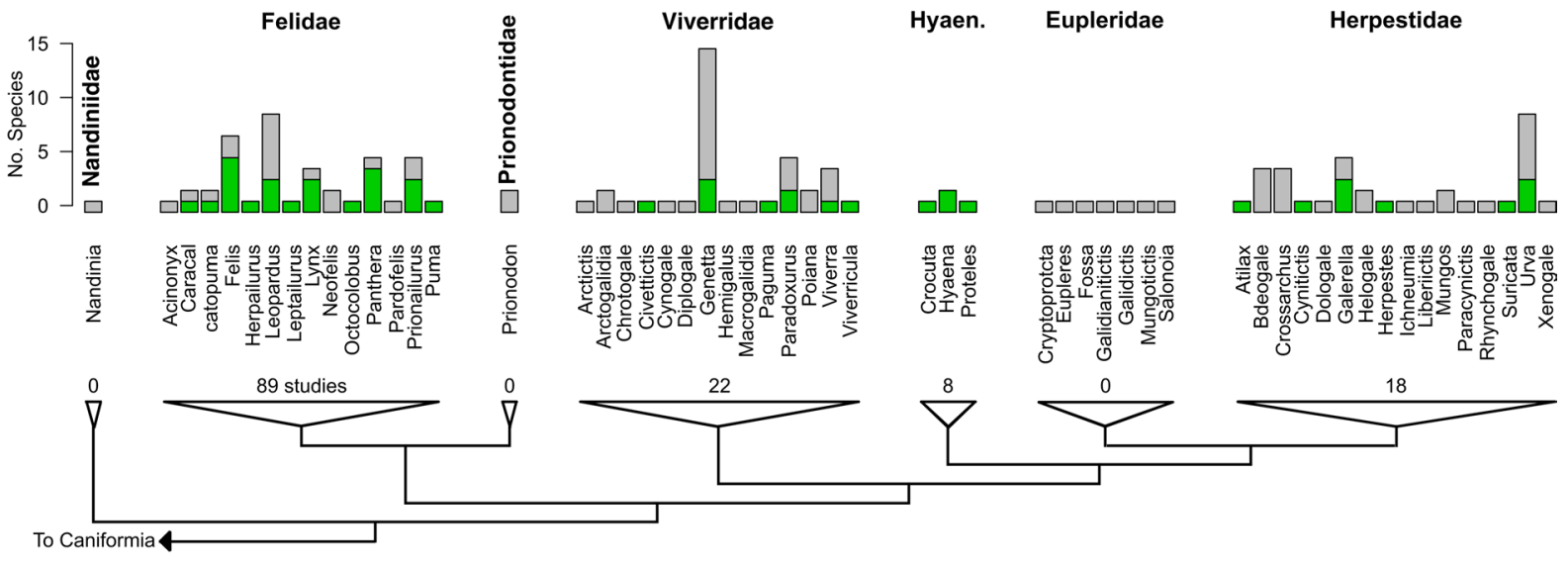

Fig. 1 - Family-level phylogeny of the Carnivora (adapted from NYAKATURA \& BININDA-EMONDS 2012) with the number of species in each genus (JACKSON et al. 2017; BURGIN et al. 2018), and the number of species with evidence of fibrous plant tissue (FPT) consumption in green. The number of studies reporting evidence of FPT consumption in each family is given beneath the list of genera. Asterisks denote families well known to consume FPT, for which the number of studies anent FPT is not given. 
(excluded from the preceding totals), the only studies identified were too equivocal to conclude if the species had ever eaten any FPT; these species are: Galictis cuja, Herpestes ichneumon, Ictonyx striatus, Leopardus wiedii, Melogale moschata, Neovison vison, and Procyon cancrivorus. For Leopardus and Procyon, other species of these genera were noted to ingest FPT while no other species were included for the other five genera (Galictis, Herpestes, Ictonyx, Melogale, and Neovison). Thirty-eight studies quantifying data on diet mentioned the occurrence of FPT but excluded it from the data collection. No pertinent studies were found for the Caniformia family Odobenidae, and none were found for the Feliformia families Eupleridae, Nandiniidae, and Prionodontidae.

Evidence for FPT consumption in the Carnivora was primarily derived from studies on scats or gastrointestinal tracts. Species with the most studies included here were Canis latrans (28 studies included here), Canis lupus (27), Vulpes vulpes (22), Puma concolor (15), Leopardus pardalis (14), Felis catus (13), Canis aureus (10), Canis familiaris (10), and Chrysocyon brachyurus (10). Apart from the Ailuridae and Ursidae, direct observations of wild animals feeding on FPT were observed in Canis familiaris (Butler \& DU ToIt 2002), Canis lupus (Murie 1944), Canis mesomelas (Hiscocks \& Perrin 1987), Crocuta crocuta (Henschel \& SKINNER 1990), Cynictis penicillata (ZumPT 1968), Leopardus pardalis, Panthera onca, and Puma concolor (Montalvo et al. 2020).

FPT consumption was found in the following Caniformia families and genera: the Ailuridae (1/1 genera, 2/2 species), including Ailurus (2/2 species); the Canidae (12/12 genera, 30/39 species), including Atelocynus (1/1 species), Canis (7/9 species), Cerdocyon (1/1), Chrysocyon (1/1), Cuon (1/1), Lycalopex (3/6), Lycaon (1/1), Nyctereutes (2/2), Otocyon (1/1), Speothos (1/1), Urocyon (2/2), and Vulpes (9/12); the Mephitidae (3/4 genera, 3/12 species) including Conepatus (1/4), Mephitis (1/2), and Spilogale (1/4); the Mustelidae (10/23 genera, 16/63 species), including Arctonyx (1/3), Gulo (1/1), Lontra (2/4), Lutra (1/2), Lutrogale (1/1), Martes (4/8), Meles (1/4), Mustela (3/17), Pekania (1/1), and Taxidea (1/1); the Otariidae (3/7 genera, 5/15 species), including Arctocephalus (3/8), Otaria (1/1), and Zalophus (1/2); the Phocidae (9/14 genera, 10/18 species), including Cystophora (1/1), Erignathus (1/1), Halichoerus (1/1), Leptonychotes (1/1), Lobodon (1/1), Mirounga (1/2), Pagophilus (1/1), Phoca (2/2), and Pusa (1/3); the Procyonidae (3/6 genera, 3/14 species), including Bassariscus (1/2), Nasua (1/2), and Procyon (1/3); and the Ursidae (5/5 genera, 8/8 species), including Ailuropoda (1/1), Helarctos (1/1), Melursus (1/1), Tremarctos (1/1), and Ursus (4/4).

Among the Caniformia, it is well known that the Ailuridae primarily feeds on bamboo grass. Consumption of grass or other FPT was observed in all genera of the Canidae and $75 \%$ of its species from 153 studies. Three studies excluded FPT consumption from data collection in Nyctereutes procyonoides.

From six studies on the Mephitidae, unidentified plants were relatively frequent for three species: Conepatus chinga, Mephitis mephitis, and Spilogale putorius. In 50 studies, grass and unidentified plants were found for some species of the Mustelidae. Needles of Pinaceae were common in one study on Pekania pennanti.

Among the Otariidae, eight studies were identified. Algae and Phyllospadix were commonly consumed by three species of Arctocephalus; algae consumption was reported less often in Otaria bryonia and Zalophus californianus. Fourteen studies documented algae consumption in some species of the Phocidae; algae consumption was detected in pups and juveniles of Pagophilus groenlandicus and two species of Phoca.

Bassariscus astutus (Procyonidae) showed evidence of FPT consumption in seven studies and in one study conifer ingestion was frequent. In two studies on Procyon lotor, grass ingestion was apparently quite common. Plant eating is well characterized for most species of the Ursidae, except for the following 
observations. Consumption of leaves and sticks was documented in Helarctos malayanus. Among three studies, FPT consumption was uncommon for Melursus ursinus. Data from one study showed Ursus arctos ingested several different species of fungi.

FPT consumption was found in the following Feliformia families and genera: the Felidae (11/14 genera, 24/42 species), including Caracal (1/2 species), Catopuma (1/2), Felis (5/7), Herpailurus (1/1), Leopardus (3/9), Leptailurus (1/1), Lynx (3/4), Otocolobus (1/1), Panthera (4/5), Prionailurus (3/5), and Puma (1/1); the Herpestidae (6/16 genera, 10/36 species), including Atilax (1/1), Cynictis (1/1), Galerella (3/5), Herpestes (1/1), Suricata (1/1), and Urva (3/9); the Hyaenidae (3/3 genera, 4/4 species), including Crocuta (1/1), Hyaena (2/2), and Proteles (1/1); and the Viverridae (6/14 genera, 9/37 species) including Civetticitis (1/1), Genetta (3/15), Paguma (1/1), Paradoxurus (2/5), Viverra $(1 / 4)$, and Viverricula $(1 / 1)$.

Among the Feliformia, 89 studies provided evidence of FPT consumption in the Felidae, covering most of the felid genera and about $57 \%$ of its species. Several studies excluded grass from data collection for the genera Herpailurus, Leopardus, Leptailurus, Panthera, and Puma. Consumption of grass and unidentified plants was evidenced in 18 studies on the Herpestidae. From eight studies, FPT consumption was relatively common in all four species of the Hyaenidae. From 22 studies, consumption of grass and other plants was detected in about $24 \%$ of the species of the Viverridae.

Grass was the most frequently observed FPT consumed, and usually it was found in relatively small amounts. Kept as pets, several studies on Canis familiaris and one study on Felis catus found grass ingestion was common. In captivity, Chrysocyon brachyurus (BARBOza et al. 1994) was observed to eat grass. Several other species in captivity were noted to eat grass, but it was unclear if evidence was based on the direct observation of feeding behaviour and/or samples of scat and vomit (BucK in LoNSDALE 2001).

A high FO (50-100\%) of grass consumption was documented in the Canidae for Canis latrans (ELLIs \& Schemnitz 1958; Holle 1973; Best et al. 1981), Canis mesomelas (Rowe-Rowe 1983), Cerdocyon thous (De Araujo 2008), Chrysocyon brachyurus (ARAGONa \& SETZ 2001), Nyctereutes viverrinus (Matsuo \& OChiai 2009), Panthera tigris (Sunquist 1981), Otocyon megalotis (Klare et al. 2011b), Speothos venaticus (Lima et al. 2009), Vulpes chama (Bотнмa 1966), and Vulpes vulpes (StePKovitch 2017). High FOs were also observed in Taxidea taxus (Mustelidae; Sovada et al. 1999), Herpailurus yagouaroundi (Felidae; BISBAL 1986), Genetta tigrina (Viverridae; RoBERTs et al. 2007), and Paguma larvata (Viverridae; Matsuo \& OCHIAI 2009). Several other studies on the Carnivora showed a high FO of FPT (Table 1) but failed to elaborate if grasses were observed, while other studies lumped grasses with other categories such as fruit, preventing an understanding of the frequency of grass ingestion.

In some cases the grass consumed was notably in large amounts, e.g., for Canis latrans (ELLIS \& Schemnitz 1958; Holle 1973; Stoel 1976), Canis lupus (Gade-Jørgensen \& StagegaARd 2000; Vos 2000; Valdmann et al. 2005), Cerdocyon thous (DE Araujo 2008), Genetta tigrina (RoberTs et al. 2007), Gulo gulo (MyHre \& Myrberget 1975), Hyaena brunnea (FAure et al. 2019), Martes americana (Marshall 1946; Francis 1958), Martes martes (Lockie 1961), Panthera pardus (HopPeDominiK 1988), Panthera uncia (Oli et al. 1993), Puma concolor (ToweILl \& MASER 1985), Vulpes chama (Bотнма 1966), and Vulpes vulpes (HATFIELD 1939; Scott 1942). In other studies on Panthera uncia, large amounts of plants were ingested which may have been grass, but this was not clarified (WeGge et al. 2012; DevKota et al. 2013).

Consumption of marine plants, bryophytes, conifers, dicots, and fungi was infrequently observed. Algae were consumed by Lontra canadensis (Buzzell et al. 2014), Ursus arctos (KISTCHINSKI 1972), U. maritimus (Russell 1975; StemPNiEwicz 2017), Vulpes lagopus (FAY \& StePHENson 1989; KaPel 
1999; Pagh \& Hersteinsson 2008), and several members of the families Otariidae and Phocidae. Arctocephalus townsendi consumed the marine plant Phyllospadix (Zosteraceae) (AurIOLES-GAMBOA \& CAMAcho-Ríos 2007). Conifer FPT were consumed by Bassariscus astutus (AleXANDer et al., 1994), Canis latrans (Santana 2010; Souther \& Wiggers 2012; Santana \& Armstrong 2017), Canis lupus (Thompson 1952; Śmietana \& Klimek 1993; Andersone 1998; Müller 2006), Lutra lutra (Bouroş \& Murariu 2017), Lynx rufus (Rollings 1945), Martes foina (ApÁthy 1998), Martes martes (Pullianinen \& OllinmäKi 1996), and Nyctereutes procyonoides (Elmeros et al. 2018).

Mosses were consumed by Bassariscus astutus (AleXander et al. 1994), Canis latrans (SANTANa \& Armstrong 2017), Cerdocyon thous (Pedó et al. 2006), Urocyon cinereoargenteus (Hatrield 1939), and Ursus maritimus (Russell 1975; GormeZano \& RocKwell 2013; STEMPNIEwICZ 2017).

Dicot species were consumed by the Ursidae. About $25 \%$ of the scats of Cerdocyon thous contained FPT of the Mimosoideae (Pedó et al. 2006). In an unknown FO, Berchemia was found in Civetticitis civetta scats (GuY 1977). Leaves of Quercus were in 14\% of scats of Canis anthus (EDDINE et al. 2017). Leaves of Betula, Fraxinus, and Quercus were in 8\% of Canis aureus scats (Stoyanov 2012). With an unknown FO, leaves of Fagus were found in Canis lupus scats (ŚMIETANA \& KLIMEK 1993). In $45 \%$ of the scats of Panthera uncia, FPT of Myricaria were detected (JumabaY-Ululu et al. 2014). Salix was found in 10\% and Tamarix was found in 17\% of scats of Genetta genetta (SÁnchez et al. 2008). Euclea leaves were ingested by Felis libyca (STUART 1976b). About 5\% of the feeding observations of Canis mesomelas observed the consumption of succulent FPT of Arthraerua, Psilocaulon, and Zygophyllum (HISCOCKs \& Perrin 1987). Cynictis penicillata apparently feeds on the succulent Chortolirion (ZumPT 1968). With an unknown FO, Oxalis bulbs were ingested by Otocyon megalotis (STUART et al. 2003).

Ingestion of fleshy, non-lichenized fungi such as mushrooms or truffles were noted in nine species: Herpestes ichneumon (Delibes et al. 1984), Martes foina (Delibes 1978), Martes martes (Pullianinen \& OllinMÄKi 1996; Helldin 2000), Meles meles (Roper \& Mickevicius 1995; Hipólito et al. 2016), Mustela erminea (BELYK 1962), Pekania pennanti (GrENFEll \& FASENFEST 1979; ZielinsKi et al. 1999), Ursus arctos (MatTson et al. 2002), Ursus maritimus (Russell 1975; GoRMEZAN \& Rockwell 2013; STEMPNIEWICZ 2017), and Vulpes vulpes (BAKALOUDIS et al. 2015).

Lichen ingestion was noted in five species of the Carnivora: Ailurus fulgens (PANTHI et al. 2012; SHARMA et al. 2014), Bassariscus astutus (AleXander et al. 1994), Canis mesomelas (Hiscocks \& Perrin 1987), Martes americana (Marshall 1946; Bull 2000), and Vulpes lagopus (PaGH \& Hersteinsson 2008).

Soils or rocks were consumed by Bassariscus astutus (AlEXANDER et al. 1994), Canis latrans (HAIGHT 1937; Bond 1939), Canis lupus (KuYt 1969), Gulo gulo (vAn DiJK et al. 2007), Lynx canadensis (Hanson \& Moen 2008), Panthera pardus (Andheria et al. 2007), Panther tigris (Powell 1957: 211; Schaller 1967: 280; Sunquist 1981; Johnsingh 1983; Khan 2008), Procyon lotor (Thompson 1952), and Vulpes vulpes (Hamilton, JR. et al. 1937; Eadie 1943; Wilson \& Dookia 2019).

\section{Discussion}

The consumption of leaves or other FPT is widespread in the Carnivora, occurring in at least 124 species (ca. $41 \%$ of the Carnivora species). Eight of these species are in the Ailuridae and Ursidae, the only two families generally considered to contain folivores that serve their nutritional needs from FPT consumption. The other 116 species are carnivores and omnivores that are not known to derive nutrition from folivory; their consumption of FPT is here supported by 344 studies. The majority of the studies concerned the Canidae (153 studies), the Felidae ( 89 studies), and the Mustelidae (50 studies). It is noteworthy that FPT consumption was found not only in omnivores and herbivores of the Carnivora, 
but also in many predominantly carnivorous species such as those of the Felidae. While the consumption of FPT serves the nutritional needs of the Ailuridae and Ursidae, the purpose of this behaviour in other species of the Carnivora, for the most part, can only be speculated. The consumption of grasses and sedges, marine plants, conifers, bryophytes, dicots, fungi, and soil by species of the Carnivora is most often likely a deliberate behavior. Sometimes these materials were ingested relatively frequently and sometimes in relatively large amounts.

\section{Grasses \& Sedges}

Grasses (Poaceae) were the most frequently consumed FPT among the Carnivora, being documented in 95 species and one hybrid of the Carnivora (Table 1). Sedges (Cyperaceae) were identified in a few studies but they may have been overlooked in other studies because of their resemblance to grasses. Regardless of the possible confusion between grasses and sedges, grasses are likely ingested more often than sedges in the Carnivora, given that several studies identified the grass genera consumed, but rarely were genera of Cyperaceae indicated (Bотнма 1966; KoK \& Nel 1992). Presumably the studies included here were reporting observations of the leaves or stems of grasses and sedges, unless their roots or seeds were specifically noted (Table 1).

While grasses are a staple food in some species (of the Ailuridae and Ursidae), in other instances, ingested grass leaves may serve to expel intestinal parasites, which is supported by observations from nine studies on eight species of the Carnivora. The earliest insight into this phenomenom may be that of MurIE (1944), who observed that some scats of Canis lupus contained both grass and roundworms (presumably Toxocaridae). Murie thought that the grass may act to scour and remove the parasites. One scat of Panthera tigris contained both grass and tapeworms (SCHALLER 1967: 280), and Schaller noted the similarity to Murie's earlier observation. KUYT (1969) found one fresh scat of Canis lupus consisting of a solid mass of grass containing tapeworms (Taenia). One scat of Cuon alpinus had two different kinds of plants, grass and the leaves of Lantana, that were together mixed with three tapeworms (Taenia) and mucus (JohNSINGH 1983). ToweIll \& MASER (1985) observed that some scats of Felis concolor consisted almost entirely of grass entwined with tapeworms. GILBERT (in HufFMAN 1997) observed in the fall, before hibernation, mature Carex spp. being consumed by Ursus arctos and subsequently the scats being composed of masses of long tapeworms. MAKUNDI (in HUFFMAN \& CATON 2001) reportedly observed the expulsion of Ascaris toxicara roundworms after dogs (Canis familiars) consumed grass. Su et al. (2013) found a significant correlation between co-occurrences of grass and Toxocara paradoxura in the scats of Viverricula indica, also providing photographic evidence (Su et al. 2013: fig. 4). Similarly, LaURIMAa et al. (2016) found a statistically significant positive correlation between infection with helminths (particularly trematodes) in Nyctereutes procyonoides and consumption of FPT, mostly grasses.

A few other studies hint at a possible relationship between intestinal parasites and grass consumption in the Carnivora. With about $72 \%$ of 50 stomachs of Lynx rufus containing intestinal parasites, it was also observed that grass and white cedar leaves occurred in most of their stomachs (RoLLINGS 1945). Urban populations of Canis latrans that had higher intestinal parasite species diversity also consumed vegetation more often (probably grasses but this was not clarified), compared to non-urban populations with lower parasite diversity that consumed vegetation less often (MANNING 2007). In the scats of Otocolobus manul, the rates of parasite frequency and grass frequency were very similar, but it was not indicated if these were correlated (Ross 2009).

The ingestion of grasses and sedges may serve to both 1) irritate and dislodge intestinal parasites and 2) stimulate gastric motility and secretion (Huffman \& CaTON 2001; McLennan \& HuffMan 2012). The morphological features of grasses and sedges that help to stimulate the gastrointestinal tract and expel 
parasites are probably the hardened epidermal serrations and trichomes that are mineralized with silica (Mehra \& Sharma 1965; Lanning \& Eleuterius 1989; Trembath-Reichert et al. 2015). Simpson (1902) stated that cats (Felis catus) "always prefer the coarser kind of grass." RoBINETTE et al. (1959) observed that Puma concolor ingested coarse grasses like Elymus condensatus which even livestock avoid in the winter, reinforcing that it is not the nutritional value of the grass that is important to Puma concolor. Hoppe-Dominiк (1988) noted that of the 30 most frequent grass species in the region, Panthera pardus chose to ingest the hairiest two species of grasses. Su et al. (2013) described the ingested grasses as all sharp-edged and covered with trichomes. Additionally, Lantana (Verbenaceae) leaves can be strongly scabrous, and were found together with grass and tapeworms in the scats of Cuon alpinus (JoHnsingH 1983). The scats of Canis anthus and Genetta genetta both reportedly contained long leaf blades of Ameplodesmos mauritanicus (BoukHERoufa et al.2020), the blades of which are rather tough and strongly serrated (ANDERSON \& Sigaut 2014). Montalvo et al. (2020) observed three species of wild Felidae consuming Oryza latifolia, which is replete with prickles on the leaf blades (SÁNCHEZ et al. 2003). Outside of the Carnivora, other animals appear to favor hairy plant tissues to aid parasite expulsion, e.g., in Ansur caerulescens (snow goose; Holmes in HuFFMAN 1997), Hylobates lar (gibbon; Barelli \& Huffman 2016), and Pan troglodytes (chimpanzee; Wranghman \& Nishida 1983; Huffman et al. 1996; Fowler et al. 2007; MCLenNAN \& HufFMAn 2012). This behaviour is possibly replicated in some marsupials that show evidence of grass ingestion, in three species of Dasyurus (GREEN 1967; Blackhall 1980; Glen \& Dickman 2006, 2008; Glen et al., 2009), Didelphis virginiana (opossum; WoOd 1954; Hopkins \& Forbes 1980), and Sarcophilus harrisii (GreEn 1967).

That the morphological features of grasses or sedges may help to expel parasites in the Carnivora is further supported by the observation that their leaves are often swallowed as large fragments instead of being finely chewed, suggesting they are not being consumed for digesting and assimilating nutrients. Among the studies included on the Carnivora (Table 1), ingested grass was described as undigested (Toweill \& Maser 1985; Hoppe-Dominik 1988; Loveridge \& MaCdonald 2003; Bekele et al. 2008; BošKović et al. 2013), in well-ordered bundles (GADE-JørGENSEN \& STAGEGAARD 2000), bundled whole (Su et al. 2013), in wads (Murie 1935; SNEAD \& Hendrickson 1942; THOMPSON 1952), in short lengths (LindSAY \& MACDONALD 1986), long blades (HAIGHT 1937), or intact (BARBOza et al. 1994; ChuANG \& LEE 1997; ChUA et al. 2016). Other studies noting grass or sedge ingestion (Table 1) generally gave no further description of the plant tissues observed. In the marsupial Dasyurus viverrinus, ingested grass blades to $5 \mathrm{~cm}$ long were described as common (BLACKHALL 1980).

Usually, the amount of grass ingested by the Carnivora was noted to be in relatively small amounts and often the FO was not very high (Table 1). Nonetheless, even rare events of grass ingestion may be purposeful in the Carnivora. For example, the earliest known observed association between parasite expulsion and grass ingestion in the Carnivora found the FO of grasses and sedges to be only about $2 \%$ for 1,174 scats of Canis lupus (MURIE 1944). On the contrary, in some studies of scats or gastrointestinal contents, grass ingestion was very frequent, with an FO of 50-100\%. Further, in other studies the amount of grass found in a single scat or stomach was notably large, sometimes comprising nearly the entire scat or stomach contents (see Results; Table 1). Possibly, small amounts of grass are ingested occasionally for prevention or control of small-scale infestations of intestinal parasites, while a larger amount or more frequent consumption of grass could be indicative of heavier or more persistent parasite loads.

Grass eating may be an innate behavior in some species of the Carnivora (BJONE et al. 2009), as even well-cared for domestic cats (Felis catus) and dogs (Canis familiaris) that might be free of intestinal parasites often regularly consume grass (HART 2008; HART \& HART 2018; HART et al. 2019). A long-held belief is that grasses are consumed by cats or dogs to alleviate nausea or induce vomiting (HUIDEKOPER 1895; Cameron 1927; Powell 1957: 210; Beaver 1981; Bush 1995; Cannon 2013). CulPePer (1666: 89) wrote that "when [dogs] are sick [...] they will quickly lead you to [dogs-grass]" which presumably 
refers to Elymus repens, a grass that can be pilose and scabrous (SzCZEPANIAK 2009). Possibly, symptoms of an illness or nausea might sometimes correlate with intestinal parasite infection (ZANZANI et al. 2014). Other historic references associate grass ingestion by dogs with vomiting (LinNAEUs 1758: 39, "Vomitu a gramine purgatur"; MoRell 1774: "Hound grass" under "Canaria"; Bоoth 1835: 290; PAUlini 1834: 29, "sic canis gramen masticando vomit, luppus a fungo purgatur"). FENN (1790) wrote that dogs eat grass to vomit, but for cats Fenn stated only that they eat grass as medicine. Recent studies show that when domestic cats or dogs consume grasses or other vegetation, they usually do not vomit nor appear to the owners to be nauseous (Sueda et al. 2007; HART 2008; BJone et al. 2009; McKenZiE et al. 2010; HART et al. 2019). DUDLEY (1892: 87) also noticed that dogs frequently ate grass without vomiting, but rather suggested that grass ingestion prevented vomiting.

Detailed quantitative data collected in controlled conditions found that vomiting is quite rare following grass ingestion in domestic dogs (BJONE et al. 2007, 2009), while more subjective reports from surveys to pet owners give a sense that vomiting is more frequent (SUEDA et al. 2007; HART \& HART 2013; HART et al. 2019). From direct observations of 2,108 total feeding events on grass by 36 dogs (Canis familiaris), only 11 times (0.5\%) did a vomiting event follow (BJONE et al. 2007, 2009). From surveys, pet owners reported that vomiting after grass consumption was relatively common in about $20-30 \%$ of domestic cats (HART \& HART 2013; HarT et al. 2019) or dogs (Sueda et al. 2007). Possibly a greater amount of variables influences the rates reported in these surveys such as the belief that grass ingestion causes vomiting, a wider variety of breeds, confounding health issues, a wider variety of grass species encountered some of which may be more toxic, and the possibility of toxins like pesticides on grasses causing adverse reactions. In one case, grass ingestion by a poodle always resulted in vomiting, which allegedly was remedied with a high-fiber diet (KANG et al. 2007). MuRIE (1944) reported one incident of Canis lupus vomiting after consuming grass. LOCKHART (1997) mentioned a dog (Canis familiaris) eating a different kind of monocot, chives (Allium sp.), and vomiting afterwards, speculating it was to control parasites.

There has also been the suggestion that grass consumption may help to bind cat hair (STILL 1908), possibly to regurgitate hair balls (BARRS et al. 1999) or pass them in scat (ChAME 2003), but strong support for this claim is lacking (DonADELLI 2019). Grass was reported as a minor component of hairballs in Hyaena hyaena (ALAm \& KHAN 2015). There is some evidence that grass may help to form regurgitated pellets in vultures (PATERSON, JR. 1984; XIROUCHAKIs 2005; Houston et al. 2007).

\section{Marine Plants}

The occasional occurrence of marine plant consumption by some Carnivora species suggests that algae have some value, but whether it serves nutritional or medicinal purposes remains uncertain. Algae were the primary FPT ingested in the marine families Otariidae and Phocidae. Algae such as Fucus and Laminaria are somewhat commonly consumed by Ursus arctos (KISTCHINSKI 1972) and U. maritimus (Russell 1975; StempNiEwicz 2017). These algal genera are known to contain significant amounts of phenylpropanoids (e.g., phlorotannins) and galactolipids (Tugwell \& BRANCH 1992; Deal et al. 2003). Algae also appear to be regularly consumed by Vulpes lagopus (FAY \& STEPHENSON 1989; KAPEL 1999; PAGH \& HeRSTEINSSON 2008). The relative importance of algae for Lontra canadensis is difficult to determine since algae were grouped together with other plants (presumably Embryophyta) into one category (Buzzell et al. 2014). Perhaps the consumption of Phyllospadix (Zosteraceae), noted in 40\% of the scats of Arctocephalus townsendi (AuRioles-Gamboa \& CAMACHo-Ríos 2007), has nutritive value, as it was also noted in trace amounts in the scats of Ursus maritimus (Russell 1975). The phenylpropanoids of Phyllospadix might also be relevant to their consumption (CHOI et al. 2009). 


\section{Conifers}

Ingestion of the FPT of conifers was noted in eight species of the Carnivora. It is very doubtful that conifer leaves would support the nutritional needs of the Carnivora. The intentional consumption of conifer leaves might be due to their rich terpene content or their phenylpropanoids (KeELING \& BohlmanN 2006; FACCOLI \& SCHLYTER 2007). The use of turpentine (derived from conifers such as Pinus spp.) has been historically utilized as an anthelmintic (Mclanahan 1918; Hall 1919; Le Roux 1930), which might explain the occurrence of conifers in the gastrointestinal tracts of some species recounted below.

RoLLINGS (1945) found that about $72 \%$ of bobcats (Lynx rufus) had intestinal parasites and that grass and white cedar (Thuja) leaves were found in most of their stomachs. In the scats of Bassariscus astutus, AlEXANDER et al. (1994) noted the conifer leaves were clearly ingested but were mostly undigested. In Canis latrans, conifer leaves were considered accidentally ingested or incidentally stuck to the scat samples (SOUTHER \& WIGGERS 2012) but were recorded as rather frequent in other scat samples (Santana 2010; Santana \& Armstrong 2017). In Canis lupus, conifer leaves were considered undigestible and unintentionally ingested (ŚMIETANA \& KLIMEK 1993), were relatively frequent in scats (ThOMPSOn 1952; ANDERSONE 1998), or were considered non-food items (MüLler 2006). Conifer leaves were grouped together with other items into one category in studies on Lutra lutra (Bouroş \& Murariu 2017), Martes foina (ApÁthy 1998), Martes martes (Pullianinen \& OllinMäKi 1996), and Nyctereutes procyonoides (ElmERos et al. 2018), being considered non-food (ElMERos et al. 2018), to be consumed in winter or in mixture with other foods (АРÁTHY 1998), or to be consumed incidentally with carrion (GolightLY et al. 2006).

\section{Bryophytes}

Reports of moss consumption by the Carnivora are few. Mosses are thought to have low digestibility, even for herbivores (Prins 1982; IHL \& BARBOZa 2007). Possibly, the secondary metabolites of bryophytes, such as the terpenoids or phenylpropanoids (PETERs et al. 2018), have medicinal effects in the Carnivora, or the high concentration of essential fatty acids are nutritionally important (PRINS 1982). A few investigations have explored the anthelmintic activity of mosses (GAMENARA et al. 2001; Roldos et al. 2008; KUMARI 2015), but the pertinence to potential activity in the Carnivora requires further inquiry.

Ursus maritimus occasionally consumes mosses (Russell 1975; Gormezano \& Rockwell 2013; STEMPNIEWICZ 2017). It was implied that the bryophytes consumed by Bassariscus astutus were well masticated and heavily digested (ALEXANDER et al., 1994). Mosses were only consumed in the spring by Cerdocyon thous (PeDó et al. 2006) and were similarly infrequent in the scats of Canis latrans (SANTANa \& ARMSTRONg 2017). Perhaps the most intriguing report was that in two stomachs of Urocyon cinereoargenteus, mosses made up the entirety of the contents (HATFIELD 1939).

\section{Dicots}

Ingestion of the FPT of several dicot species was recorded in the Carnivora, having relatively significant FOs or with direct observations of their consumption, suggesting that it is likely intentional. It is difficult to speculate upon the nutritional or medicinal value of these occurrences. The Ursidae may feed on a variety of dicot leaves (CHHANGANi 2002; MCLELLAN 2011; GoRMEZANO \& RocKWELl 2013; FuRUSAKA et al. 2017; StemPNiewicz 2017; Sethy \& ChauHAn 2018), probably for nutritional purposes in many instances, but potential medicinal value cannot be summarily dismissed.

Several other FPT of dicots consumed in the Carnivora included Berchemia (for Civetticitis civetta; Guy 1977), Euclea (Felis libyca; Stuart 1976b), Fagus (Canis lupus; Śmietana \& KlimeK 1993), 
Myricaria (Panthera uncia; Jumabay-Ululu et al. 2014), Quercus (Canis anthus; Eddine et al. 2017), the Mimosoideae (Cerdocyon thous; Pedó et al. 2006), Salix and Tamarix (Genetta genetta; SÁnCHEZ et al. 2008), and Betula, Fraxinus, and Quercus (Canis aureus; Stoyanov 2012). The leaves and stems of these plants would probably be considered undigestible to these Carnivora species. The leaves of many of the above plant genera are not particularly hairy but may have significant concentrations of phenylpropanoids (such as lignin and tannins), e.g., in Berchemia (LeE et al. 1995), Betula (Wratten et al. 1984), Euclea (Maroy 2017), Fagus (Bussotti et al. 1998), Fraxinus (SchempP et al. 2000), Myricaria (Chernonosov et al. 2017), Quercus (García-Villalba et al. 2017), Salix (JulkunenTITTO 1985), and Tamarix (Ksouri et al. 2009). Terpenes and alkaloids, such as in Euclea (MAROYI 2017), may also be significant. The Mimosoideae notably contain significant amounts of alkaloids (WINK 2013).

Two canids ingested succulent plants in Africa. Canis mesomelas consumed FPT of the plant genera Arthraerua, Psilocaulon, and Zygophyllum (Hiscocks \& Perrin 1987) and Cynictis penicillata consumed Chortolirion (ZuMPT 1968). Perhaps the water content or the secondary metabolites of these plants are important to these canids.

Unknown woody material was exceptionally frequent in a study of Vulpes vulpes (STEPKOVITCH 2017). Oxalis bulbs were ingested by Otocyon megalotis (StUarT et al. 2003). As previously mentioned, scabrous Lantana (Verbenaceae) leaves were found with grass and tapeworms in a scat of Cuon alpinus (JoHnsingh 1983). It also well known that the Felidae may ingest Nepeta cataria (catnip), but their behavior appears to be primarily concerned with the odor and not the ingestion of the plant (TUCKER \& TUCKER 1988).

\section{Fungi}

Fungi are occasionally consumed by the Carnivora, probably for nutritional properties but their secondary metabolites cannot be discounted. Fleshy fungi were consumed by nine species of the Carnivora and lichens were ingested by five species (see Results).

Fleshy fungi such as mushrooms are easily masticated and nutritive substances could probably be absorbed (Claridge \& TRAPPe 2005; Urban 2016). Mushrooms were occasionally consumed by some mustelids (Belyk 1962; Delibes 1978; Pullianinen \& OllinmäKi 1996; Helldin 2000), and especially Pekania pennanti may apparently feed frequently on false truffles (Boletales) (ZIELINSKI et al. 1999). False truffles, mushrooms, and puffballs are seasonally useful to Ursus arctos (MatTson et al. 2002; Vulla et al. 2009), which may make some scats liquidy, perhaps indicating a laxative effect and perhaps partly indicative of the high water content of these fleshy fungi. Vulpes vulpes was also noted to consume mushrooms (BAKALOUDIS et al. 2015).

Lichens typically have tough thalli and presumably they would not be easily digested by the Carnivora. While lichens might provide some nutrients to carnivores (DUBAY et al. 2008), the secondary metabolites of lichens may be more relevant (NYBAKKEN et al. 2010), though little is known concerning potential medicinal or anthelmintic activity in the Carnivora. Canis mesomelas was directly observed once ingesting the crustose lichen Caloplaca (HISCOCKS \& PERrin 1987). Lichens formed the bulk of one scat of Martes americana (MARSHALl 1946). It was implied that the lichens consumed were well masticated and heavily digested in Bassariscus astutus (ALEXANDER et al. 1994). The amount of lichens consumed by Vulpes lagopus was unclear since lichens were lumped into one category with leaves, mosses, and twigs (PaGH \& HeRsteinsson 2008). 


\section{Soils}

Ingestion of soils or rocks were occasionally noted in the Carnivora. Geophagy has been speculated to alleviate gastrointestinal problems such as parasites or toxins, or provide minerals (BEYER et al. 1994; WiLSON 2003; KRISHNAMANI \& MAHANEY 2009). Like grasses or other scabrous plant tissues, rocks and soils could conceivably mechanically irritate and remove parasites. SCHALLER (1967: 255) described a scat of Panthera tigris consisting of a number of tapeworm segments and a small amount of soil. Soil ingestion appears particularly common in Panthera tigris (JoHNSINGH 1983), sometimes being noted in most of the scats and some scats having fairly large amounts of soil (Powell 1957: 211; SCHALLeR 1967: 280; SUNQUIST 1981; KHAN 2008).

EADIE (1943) noted that occasional scats of Vulpes vulpes were almost wholly soil or gravel. The presence of rocks in the scat of Lynx canadensis was stated to be "most surprising" (HANson \& MoEN 2008). Dirt was in $28 \%$ of Gulo gulo scats (vAN DiJK et al. 2007) and in $24 \%$ of Panthera pardus scats (ANDHERIA et al. 2007). Earth, gravel, pebbles, and non-grass FPT were encountered with surprising frequency in the scats and stomachs of Canis latrans (Bond 1939), and in one scat was a large amount of dirt (HAIGHT 1937). KUYT (1969) described that several scats of Canis lupus were entirely made of an unidentified material resembling dried clay, but then speculated it was undigested material from animal prey. The frequent presence of soil in the scats of Procyon lotor was thought to be obtained from the crops and gizzards of bird prey (THOMPSON 1952). Clearly, some species of the Carnivora consume soil but the reasons for this are unclear.

\section{Is ingestion of FPT accidental?}

An overwhelming majority of the 344 studies provided almost no interpretation or discussion concerning the evidence observed of FPT consumption in the Carnivora. About one-sixth of the studies suggested that the consumption of FPT by the Carnivora was accidental or incidental. In contrast, about one-sixth of the studies suggested or concluded that FPT consumption was intentional.

In the studies that favored interpreting the ingestion of FPT as unintentional, the most common explanation given was that the predator incidentally consumed the FPT present in the gastrointestinal tracts of prey. Other reasons given were that the FPT was consumed from herbivore dung, during grooming, or from material near prey. It was occasionally speculated that FPT detritus on the ground had become externally stuck to a scat. One study described "digested grass" to imply it originated from the digesta of the prey, contrasting it with the undigested grass consumed (LOVERIDGE \& MACDONALD 2003). Nonetheless, in the two Carnivora species studied, the digested grass had a FO of $2 \%$, while undigested grass had a FO of 45-47\% (LOVERIDGE \& MACDONALD 2003).

Behavioral observations indicate carnivores typically avoid the gastrointestinal tracts of prey (THOMPSON 1952; Schaller 1967; Jobin et al. 2000; BUCK in LonSDALE 2001: appendix B) or eat the tissues of the gastrointestinal tract but avoid the digesta of large herbivorous prey (JOHNSINGH 1983; FABREGAS et al. 2016). Peterson \& CiUcCI (2003: 123) stated that the digesta "is of no interest to" Canis latrans, but that they may consume the stomach lining and intestinal wall. However, WADE \& Bowns (1985) stated "the milk-filled stomach is a preferred item" for Canis latrans. Among several captive species of Carnivora, it was observed that the gastrointestinal tract and its contents are typically avoided, except it was alleged that Lycaon pictus may eat a small amount of the digesta (BUCK in LONSDALE 2001: appendix B). Nonetheless, in captivity, Lycaon pictus was also presumably observed to eat grass (BuCK in LONSDALE 2001: appendix B). Black bears (Ursus americanus) were described as "clean [...] delicate feeders [whereby] most debris is either spat out or avoided" (BACON \& BuRghardT 1976). For the Felidae, it had been stated that their "feeding pattern is relatively neat" (WADE \& Bowns 1985). As some 
smaller prey may be consumed whole (BUCK in LONSDALE 2001: appendix B), possibly digesta and FPT consumed from small prey could have been detected in carnivore scats or stomachs. The corms of a grass (Melica) in the scats of Canis latrans were thought to derive from the cheek-pouch contents of rodent prey (MURIE 1935). The consumption of seeds from prey intestines, a form of diploendozoochory, has been considered plausible, although proof that seeds have actually been consumed from prey intestines by wild animals is wanting (HäMÄLÄINEN et al. 2017).

It also been said that the ingestion of FPT or other items was due to the animals being trapped. GIPSON (1974) stated that trapped Canis latrans "tend to chew and swallow almost anything within reach" as a reason to exclude collecting data on ingestion of FPT. Similarly, in the marsupial Sarcophilus harrisii, it was explained that the animals probably chew and ingest grass while trying to escape; on the contrary, the same study implied that the evidence of grass ingestion by Dasyurus viverrinus was derived from prey, i.e., the stomach contents of wallabies (GREEN 1967).

The feeding habits of the Carnivora suggest that accidental ingestion of FPT is a poor explanation for its frequent occurrence in scats or gastrointestinal tracts of the Carnivora, especially when there is a lack of direct evidence that FPT are indeed accidentally consumed. Moreover, there are direct observations of species of Carnivora deliberately eating FPT (e.g., MonTaLvo et al. 2020; see Results). Further, it would be disadvantageous for carnivores to be imprecise in their eating habits (e.g., incidentally consuming prey digesta), which could potentially increase their exposure to infectious diseases or toxins.

\section{Conclusions}

Plant eating is widespread in the Carnivora, and includes frugivory, granivory, rhizovory, nectarivory, and folivory. Well over 100 species of the Carnivora deliberately ingest leaves or other FPT, for a variety of purposes. Grasses and sedges are especially useful to the Carnivora, in many cases ostensibly to manage intestinal parasites, as plant leaves with abrasive or hairy structures mineralized with calcium or silicon (LANNING et al. 1958; LANNING 1961; Kaufman et al. 1981; DayanANDAN 1983; LanNing et al. 1980; LANNING \& EleUterius 1989; WeIGeND et al. 2018) appear to be most sought after to mitigate intestinal infections. While control of intestinal parasites is a plausible explanation for the ingestion of abrasive or hairy plants (or perhaps soil), additional focused research is desirable to corroborate this. Fresh and old scats might both be useful to observe this potential association (NAPOLI et al. 2016).

The FPT of marine plants, bryophytes, conifers, and dicots are deliberately consumed by some species of the Carnivora, but it is unclear how it may affect their fitness. Many of these plants lack scabrous structures and probably are relative undigestibile, giving cause to consider that their secondary metabolites may have some value, such as anthelmintic properties (e.g., QuINLAN et al. 2002; KATIKI et al. 2011; NdjonKa et al. 2014; Romero-Benavides et al. 2017; Spiegler et al. 2017; Liu et al. 2020). Several other reasons were provided to explain the ingestion of FPT in the included studies (Table 1), such as the FPT acting as a food, source of minerals or vitamins, toxin elimination, water source, antiinflammatory, hair elimination, maintenance of the gastrointestinal tract during starvation, or a digestive aid (e.g., for bones, food, hair, or skin). These possibilities also bear consideration. The consumption of fungi or soil also requires further investigation to understand their role and value. The consumption of FPT, fungi, or soil could also be an exploratory behavior that does not always increase fitness. Since diet influences the gut microbiome (NISHIDA \& OCHMAN 2018), it would be of interest to explore how the consumption of FPT, fungi, or soil could influence the gut microbiome of the Carnivora.

That about 41\% (123 species) of the Carnivora may consume FPT is probably an underestimate for several reasons. First, its occurrence has definitely been underappreciated and in many cases probably 
ignored altogether. Most studies included here were focused on the dietary analysis of carnivory and frugivory, and usually showed negligible interest concerning the consumption of FPT. It is likely that many other studies on the Carnivora found FPT but never reported it. Indeed, 38 studies reported here explicitly excluded FPT evidence from data collection. Some studies stated that the evidence of FPT consumption was only recorded if there was a relatively large amount of FPT detected (e.g., EADIE 1943; SChaller 1967; ANDelt et al. 1987). Second, potential FPT consumption by other species is unknown because the ecology of many species (e.g., scat analyses) is poorly known; examples include numerous viverrids (PAPEŞ \& GAUBert 2007), Bornean felids (MOHAMED et al. 2009), the canid Vulpes pallida (Brito et al. 2009), and the mustelid Bdeogale jacksoni (De LuCA \& Rovero 2006) which are all understudied. Lastly, this study undoubtedly failed to include all pertinent studies ever published.

To better understand FPT consumption in the Carnivora, it is requisite that more attention is paid to the species, amounts, and parts of plants ingested (for exceptionally detailed analyses of consumed plant tissues in the Carnivora see Scott 1942; Thompson 1952; Alexander et al. 1994; Santana 2010: 24), as well as the health of the animal, such as intestinal parasites. The same is true for investigating the role of fungi (Claridge \& MAY 1994) and soil in the Carnivora. Even the absence of plants, soil, or fungi in scats or stomachs is useful information if it is explicitly stated these items were searched for but not found. If the methodologies are standardized and results are more detailed, then it will be possible to compare across studies and make inferences about conditions that lead to FPT, fungi, or soil consumption. Direct comparisons are not practical nor statistically logical among the 344 studies here (Table 1) because the methodologies and results are excessively heterogeneous. About half of the studies (Table 1) either nebulously defined what kinds of FPT were consumed (e.g., using the vague category "plant material" or "vegetation") or combined multiple discrete items into one category (e.g., grasses and fruits combined). Very rarely $(\sim 5 \%)$, was plant material treated in detail to identify the genera or species consumed (e.g., Scott 1942; Thompson 1952; LeVER 1959; Viljoen \& Davis 1973; Bold \& Dorzjunduy 1976; Stuart 1976b; Hiscocks \& Perrin 1987; KoK \& Nel 1992; Śmietana \& KlimeK 1993; Alexander et al. 1994; Chuang \& Lee 1997; Melville et al. 2004; Álvarez-Castañeda \& GonZÁlez-Quintero 2005; Aurioles-Gamboa \& CAMACHo-Ríos 2007; SÁnChEZ et al. 2008; NAKWAYA 2009; RAMesh et al. 2009; Su et al. 2013; Habtamu et al. 2017; AKrim et al. 2019; BoukHeroufa et al. 2019; Montalvo et al. 2020). It is realized that often times the material may be very scant or extremely difficult to identify morphologically without intensive efforts. Availability of DNA sequencing resources will certainly be useful to identify plants or fungi consumed by the Carnivora. Plastid primers were used by XIONG et al. (2016) to identify plants in the scats of Prionailurus bengalensis, although they were unable to confidently identify plants to the species level.

\section{Acknowledgements}

Special thanks to Isabelle Schön for her gracious help throughout; thanks to Urmas Saarma and the scientific editor for their helpful comments to improve the manuscript; and thanks to Kristiaan Hoedemakers for help processing the manuscript. Gratitude is extended for the support from the Department of Biological Sciences at Florida International University, the Department of Cell Biology, Microbiology and Molecular Biology at the University of South Florida, family, and friends. Alan's deepest appreciation to Kaitlin, Cormac, Fern, and especially Puppers who Alan regularly observed eating grass and began to wonder why a dog would eat grass; this study is the culmination of that inquiry. 
U .

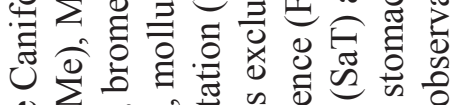

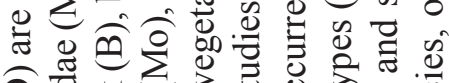

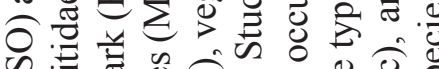

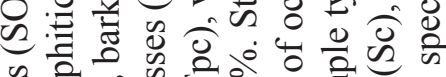

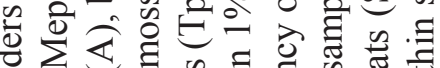

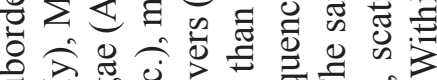

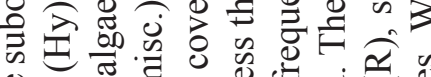

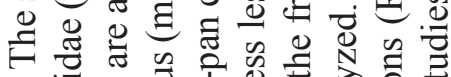

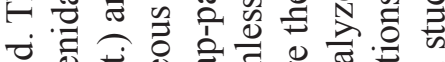

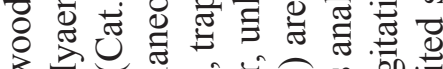

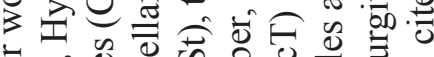

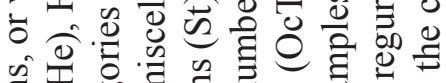

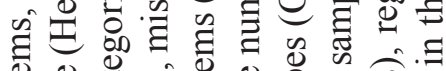
क्ष की

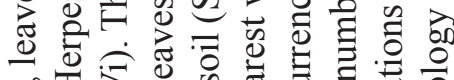

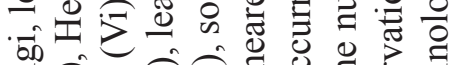

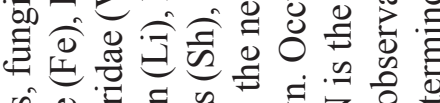

- की

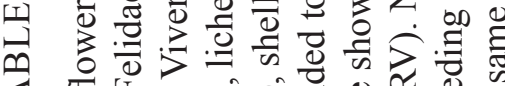

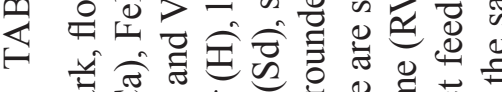
记 \% \&

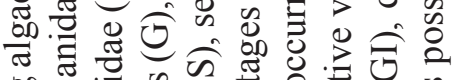

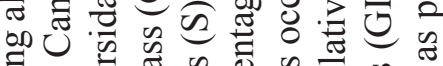
.

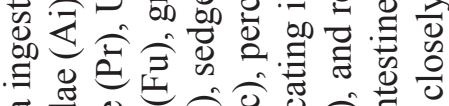

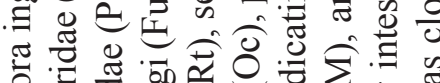

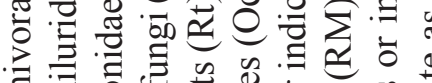

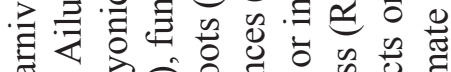

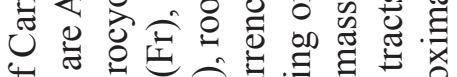

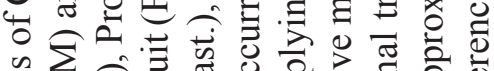

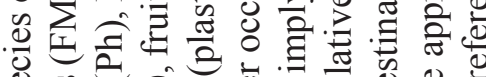

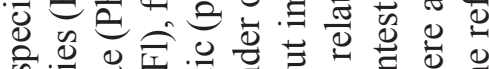

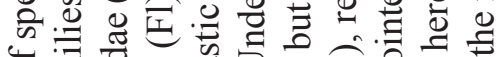

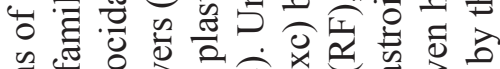

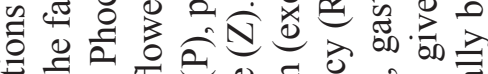
है

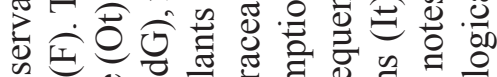

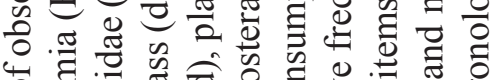

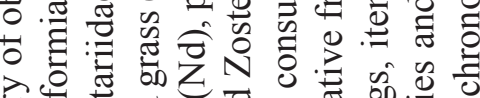

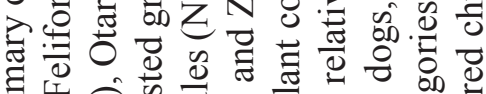

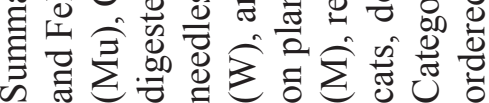

\begin{tabular}{|c|c|c|c|c|c|c|c|c|c|c|c|c|c|c|}
\hline 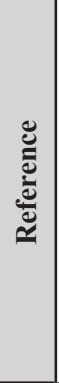 & 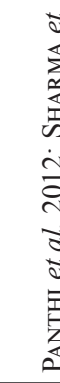 & & 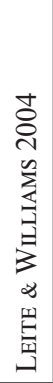 & 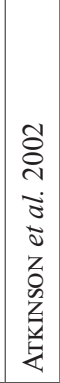 & 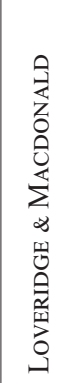 & & 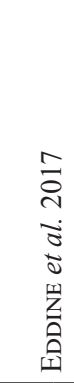 & 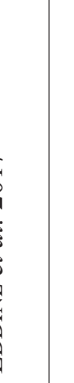 & 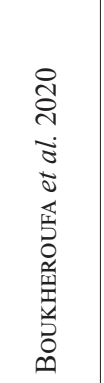 & 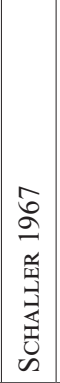 & 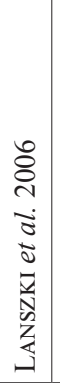 & 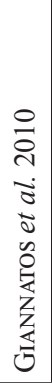 & 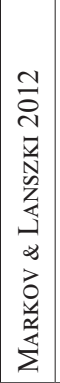 & 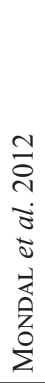 \\
\hline : & 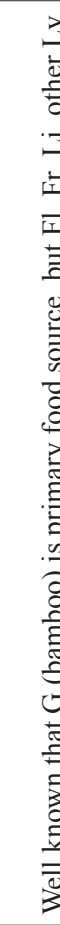 & 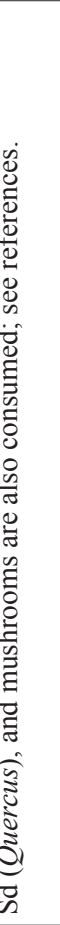 & 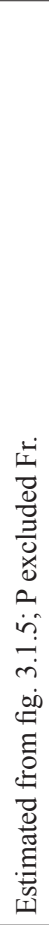 & 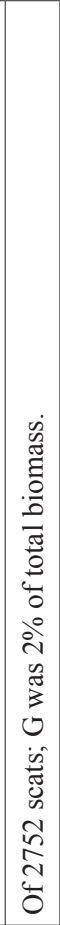 & & & 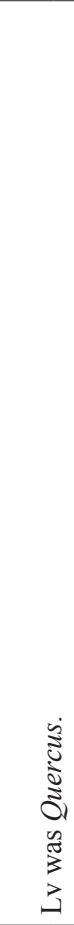 & 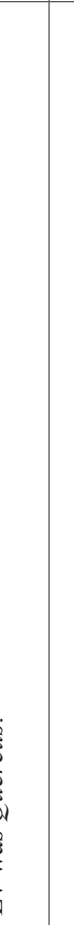 & 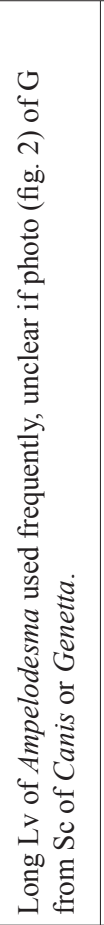 & 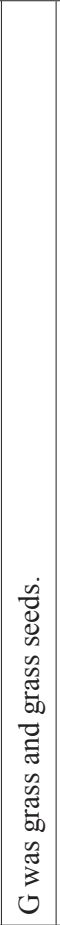 & 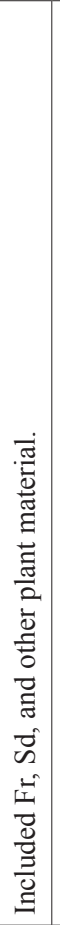 & & & 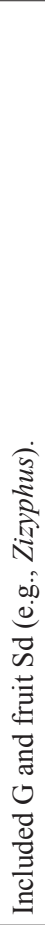 \\
\hline हू & 1 & 1 & $\ddot{n}$ & \pm & v & 品 & $\ddot{n}$ & & $\ddot{n}$ & $\ddot{\mathscr{n}}$ & $\ddot{\varkappa}$ & $\ddot{n}$ & $\ddot{n}$ & $\ddot{\mathscr{L}}$ \\
\hline z & 1 & 1 & $\vec{N}$ & 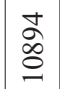 & $\ddot{2}$ & 6 & $\stackrel{0}{\sim}$ & & i & $\stackrel{\infty}{\sim}$ & $\underset{\infty}{\vec{\infty}}$ & $\widehat{\beth}$ & $\stackrel{0}{0}$ & $\stackrel{ \pm}{\circ}$ \\
\hline$\overline{\check{g}}$ & 1 & 1 & 이 & o & 8 & 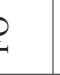 & 이 & & 1 & Oે & $\sum_{\Omega}$ & O) & O & 이 \\
\hline ర̊ & 1 & । & $\stackrel{\circ}{\dot{\sigma}}$ & $\stackrel{0}{\infty}$ & $\stackrel{\mathrm{i}}{ }$ & 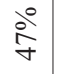 & ฉे & $\stackrel{\text { 巳े }}{\mathrm{O}}$ & I & ले & ठें & ¿ें & $\stackrel{\circ}{\grave{m}}$ & $\stackrel{\circ}{\infty}$ \\
\hline Uే & $\begin{array}{l}3 \\
3 \\
0 \\
ث\end{array}$ & $\begin{array}{l}3 \\
0 \\
\text { it }\end{array}$ & $a$ & 0 & O & 0 & 0 & ב & 0 & 0 & 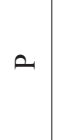 & 0 & 0 & $>$ \\
\hline $\begin{array}{l}\stackrel{\mathscr{u}}{\mathscr{J}} \\
\text { के }\end{array}$ & 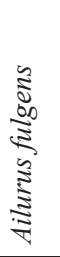 & 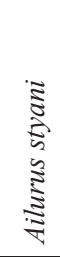 & 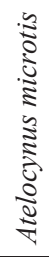 & & 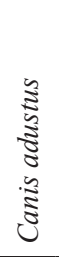 & & & 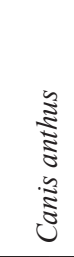 & & & & 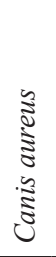 & & \\
\hline$\sum$ & \multicolumn{2}{|c|}{$\bar{z}$} & \multicolumn{12}{|c|}{$\pi$} \\
\hline O & & & & & & & U & & & & & & & \\
\hline
\end{tabular}




\begin{tabular}{|c|c|c|c|c|c|c|c|c|c|c|c|c|c|c|c|c|c|c|c|c|c|}
\hline 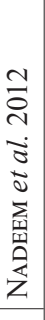 & $\begin{array}{l}\text { ̃ } \\
\overline{1} \\
z \\
z \\
\vdots \\
\vdots \\
\vdots\end{array}$ & & 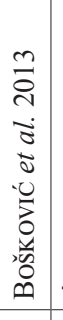 & 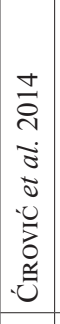 & 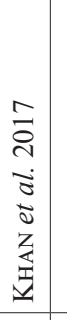 & 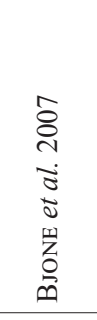 & \multicolumn{5}{|c|}{ 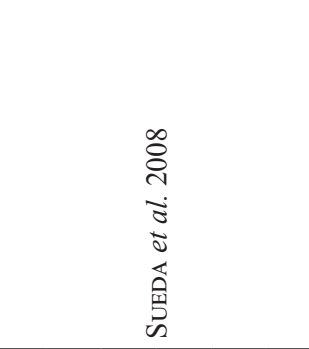 } & 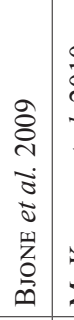 & 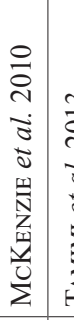 & 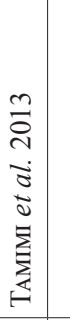 & 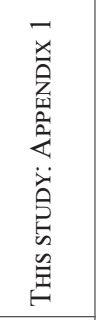 & 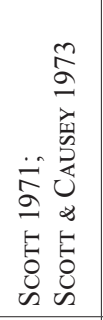 & 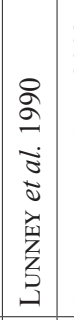 & 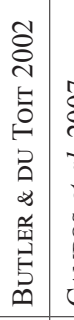 & 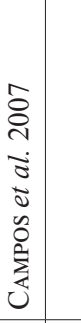 & 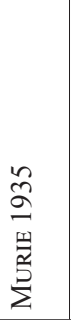 & $\begin{array}{l}\hat{\sigma} \\
\hat{\sigma} \\
\underline{E} \\
\vec{I}\end{array}$ \\
\hline 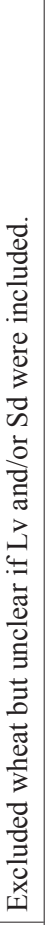 & 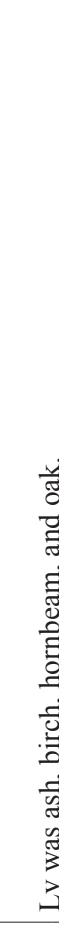 & & 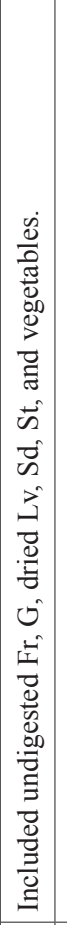 & 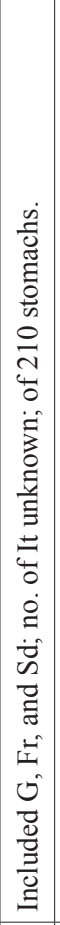 & & 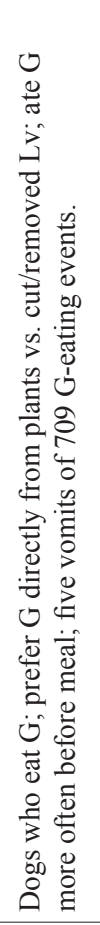 & \multicolumn{5}{|c|}{ 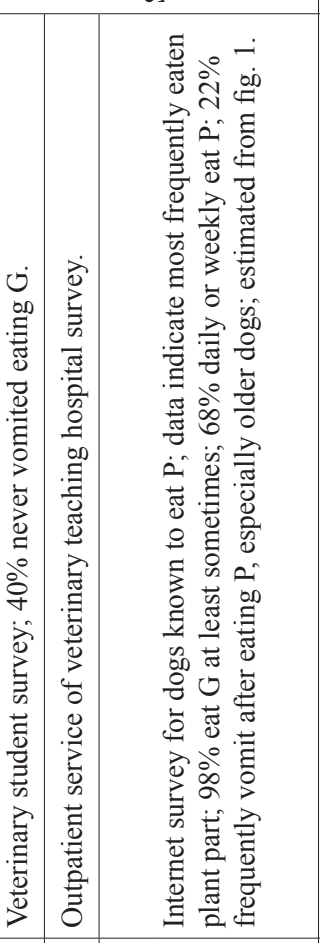 } & 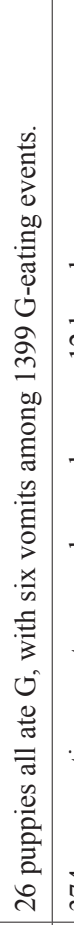 & 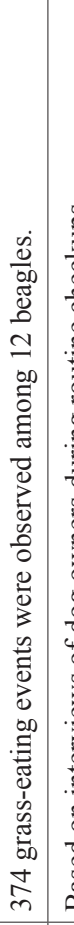 & 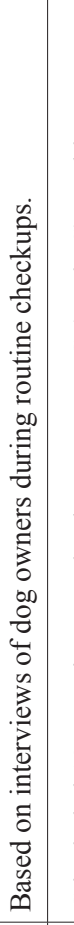 & 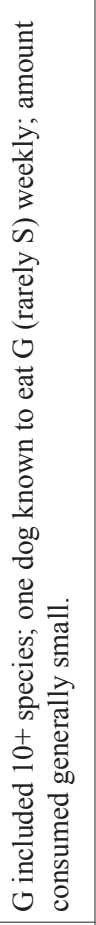 & 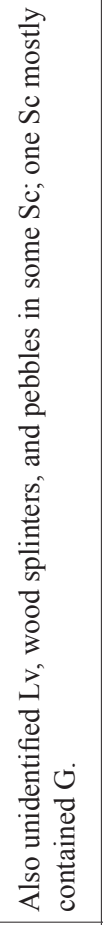 & 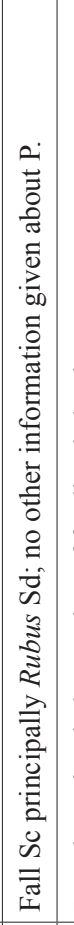 & 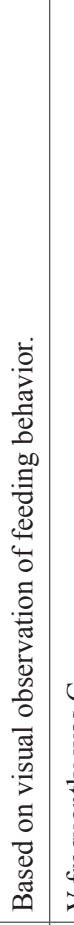 & 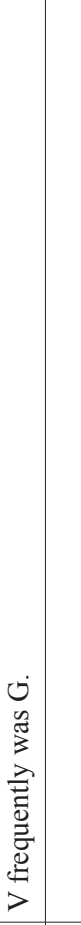 & 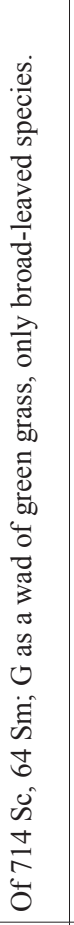 & 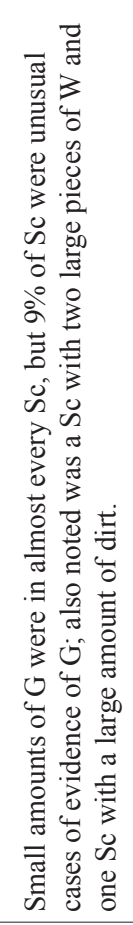 \\
\hline$\check{\check{n}}$ & है & & $\Xi$ & \pm & $\ddot{n}$ & $\begin{array}{l}0 \\
\frac{0}{0} \\
\theta\end{array}$ & $\begin{array}{l}5 \\
8 \\
8 \\
0\end{array}$ & $\begin{array}{l}10 \\
60 \\
8 \\
0\end{array}$ & \multicolumn{3}{|c|}{$\begin{array}{l}\text { ch } \\
\frac{6}{0} \\
0\end{array}$} & 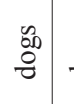 & $\begin{array}{l}5 \\
0 \\
0 \\
0\end{array}$ & $\begin{array}{l}6 \\
60 \\
0 \\
0\end{array}$ & $\begin{array}{l}6 \\
\frac{6}{60} \\
\frac{8}{0}\end{array}$ & $\ddot{n}$ & $\ddot{n}$ & 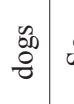 & $\ddot{n}$ & $\begin{array}{l}\text { E } \\
\text { s. } \\
\text { is }\end{array}$ & $\ddot{\mathscr{n}}$ \\
\hline$\stackrel{\infty}{\sigma}$ & 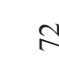 & & $\stackrel{\infty}{\approx}$ & 1 & $\tilde{\infty}$ & $\simeq$ & 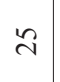 & 于 & \multicolumn{3}{|c|}{$\vec{n}$} & $\stackrel{i}{i}$ & $\simeq$ & $\stackrel{ \pm}{\grave{~}}$ & $m$ & $n$ & $\stackrel{2}{\infty}$ & $\therefore:$ & $\hat{m}$ & $\stackrel{\infty}{\vDash}$ & $\cong$ \\
\hline O & O & & o & O & 이 & 1 & o & O & \multicolumn{3}{|c|}{ 이 } & 1 & 1 & O & 1 & o & 足 & $\sum$ & $\stackrel{1}{\simeq}$ & 이 & 이 \\
\hline iे & $\begin{array}{l}0 \\
\dot{0} \\
0\end{array}$ & $\stackrel{\circ}{\infty}$ & 学 & $\stackrel{\circ}{+}$ & 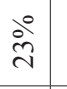 & 1 & ठें & ڤ̊ & ذి ڤे & in & $\therefore$ & I & 1 & ڤें & 1 & ১ & 号 & $\stackrel{\circ}{\vec{v}}$ & $\stackrel{0}{\circ}$ & ले & ลे \\
\hline 0 & 0 & 군 & $a$ & $a$ & 0 & 0 & ט & 2 & \begin{tabular}{l|l}
$\vec{\omega}$ & 0 \\
$n^{\prime}$ &
\end{tabular} & 3 & $\vec{\simeq}$ & ט & ن & ט & $\begin{array}{l}n \\
0\end{array}$ & 0 & $a$ & $0=$ & $>$ & 0 & 0 \\
\hline & & & & & & \multicolumn{10}{|c|}{ 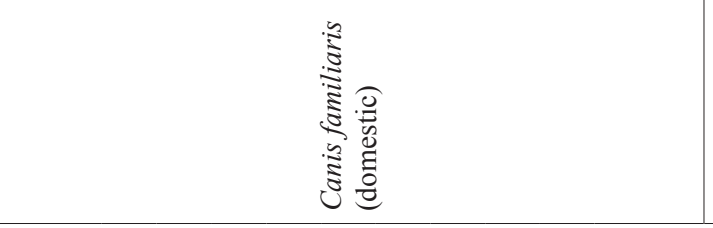 } & \multicolumn{4}{|c|}{ 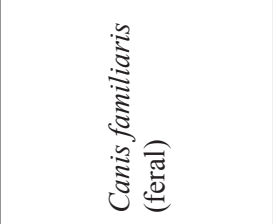 } & & 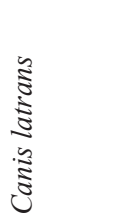 \\
\hline \multicolumn{22}{|c|}{$\tilde{U}$} \\
\hline & & & & & & & & & & & $u$ & & & & & & & & & & \\
\hline
\end{tabular}




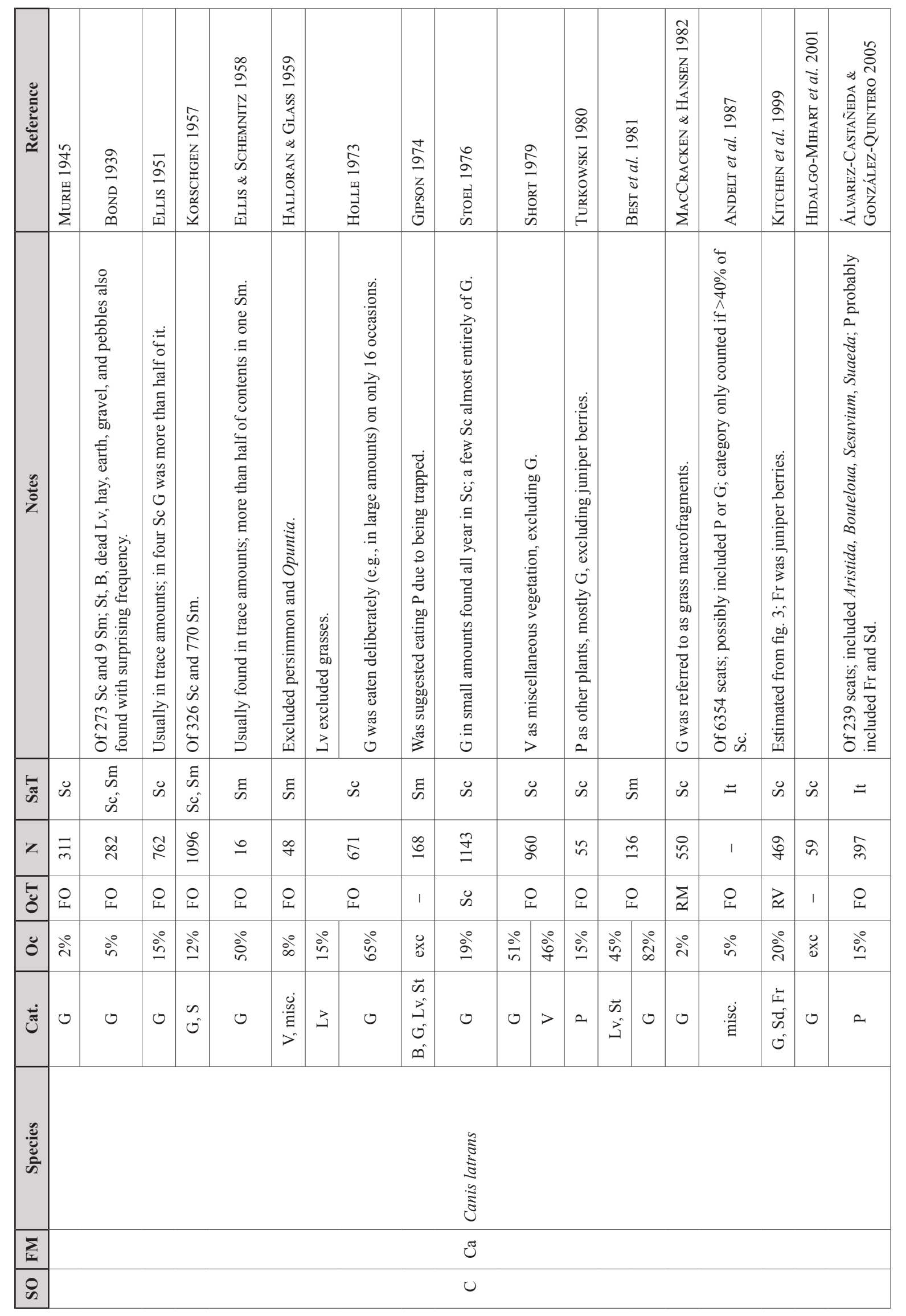




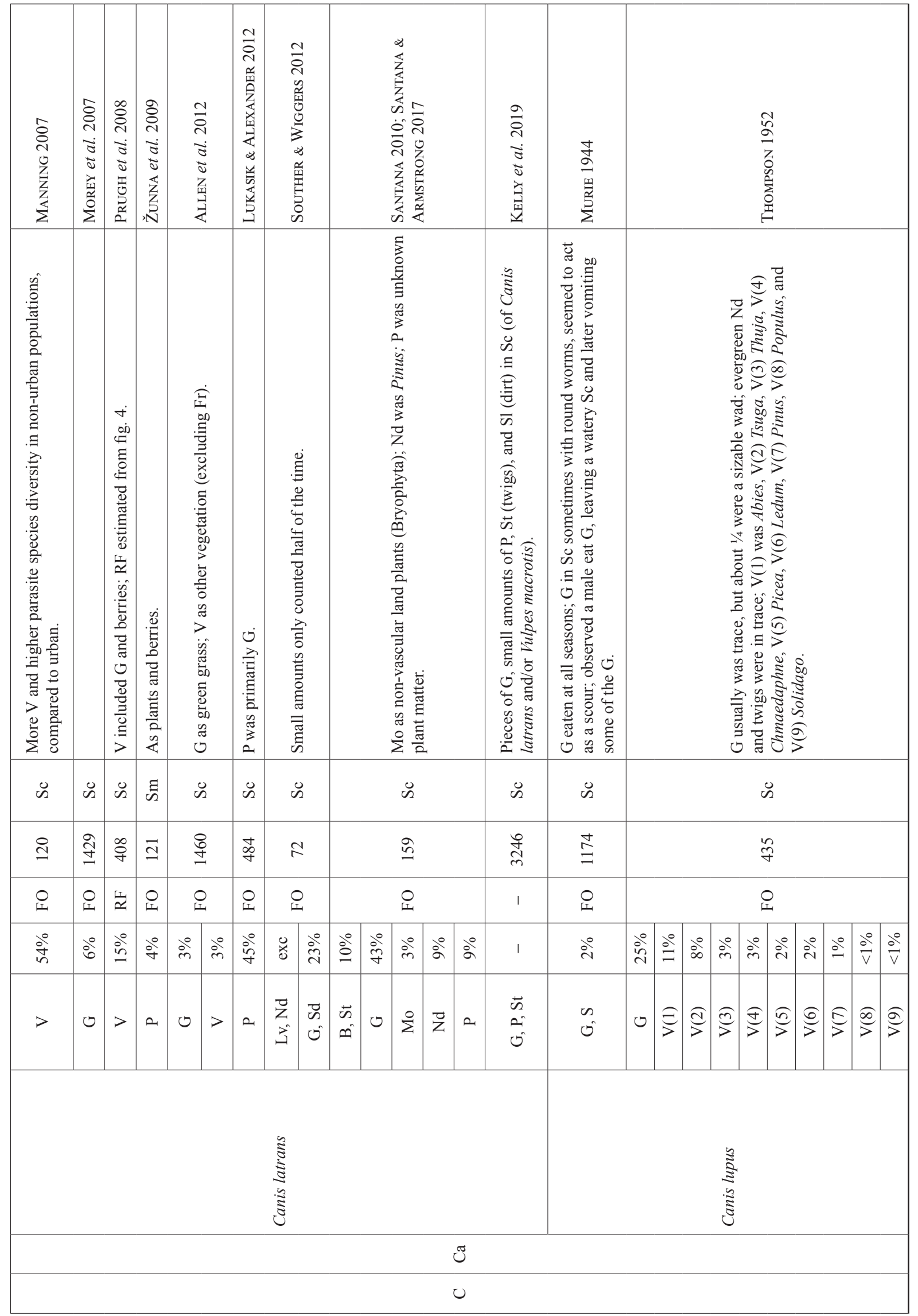




\begin{tabular}{|c|c|c|c|c|c|c|c|c|c|c|c|c|c|c|c|c|c|c|c|c|c|}
\hline 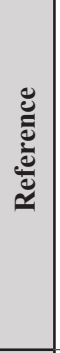 & 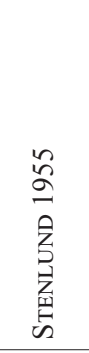 & 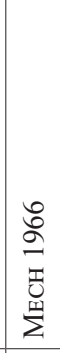 & & 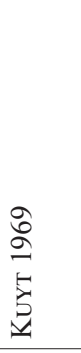 & 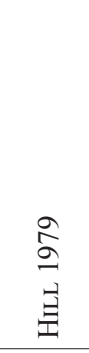 & 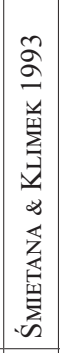 & 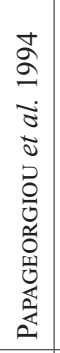 & 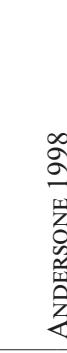 & & 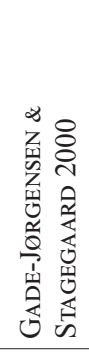 & $\begin{array}{l}8 \\
0 \\
i \\
0 \\
0 \\
>\end{array}$ & 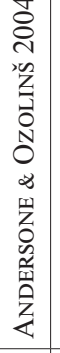 & 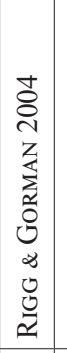 & 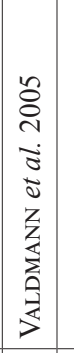 & 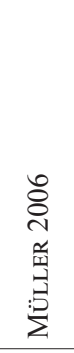 & 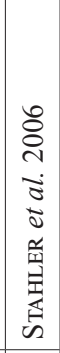 & 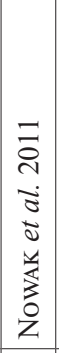 & 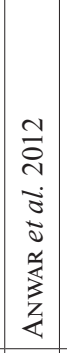 & 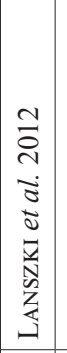 & 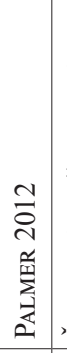 & 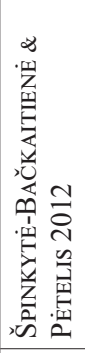 \\
\hline : & 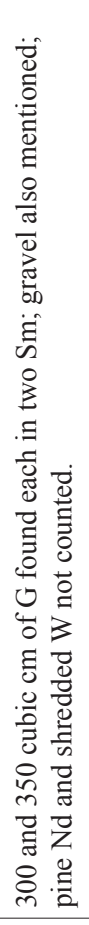 & & & 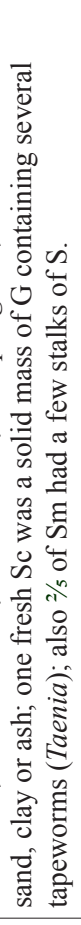 & 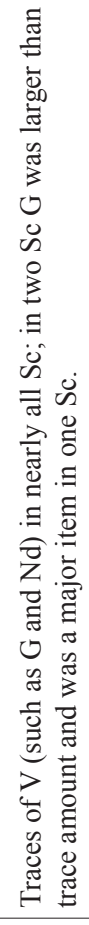 & 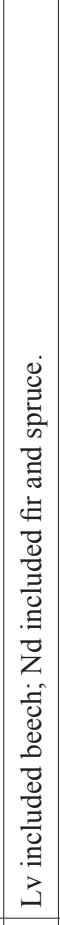 & 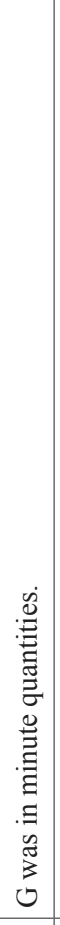 & & & 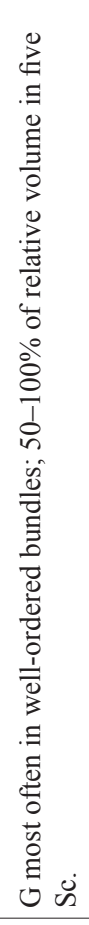 & 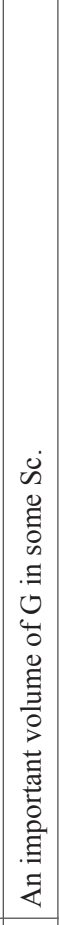 & & & 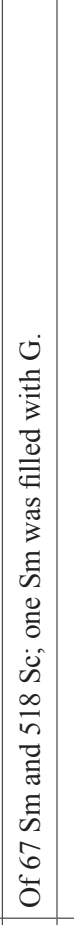 & 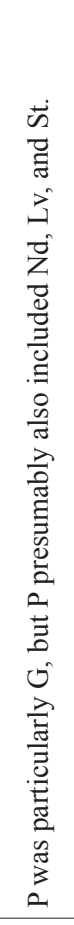 & 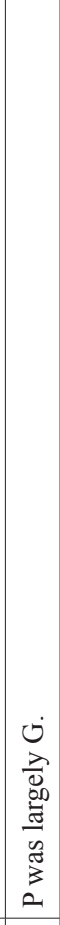 & & 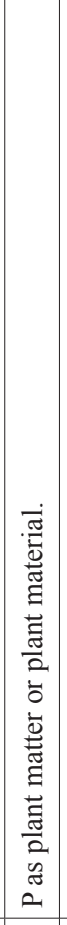 & 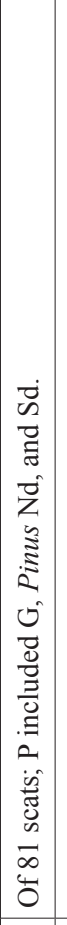 & 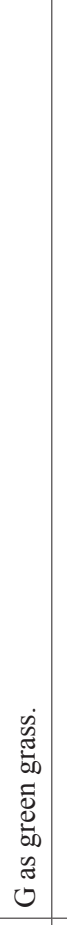 & 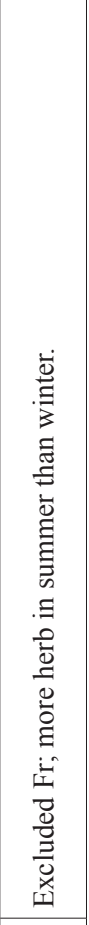 \\
\hline$\overline{\tilde{n}}$ & Е & $\ddot{n}$ & & \pm & $\ddot{n}$ & $\ddot{n}$ & हี & $\ddot{n}$ & & $\check{\curvearrowleft}$ & $\ddot{\mathscr{n}}$ & $\ddot{\curvearrowleft}$ & $\ddot{\varkappa}$ & $\begin{array}{l}\tilde{y} \\
\tilde{n} \\
\tilde{n}\end{array}$ & $\ddot{n}$ & $\ddot{n}$ & $\ddot{n}$ & $\ddot{n}$ & $=$ & $\ddot{n}$ & $\ddot{n}$ \\
\hline$z$ & 1 & $\stackrel{\infty}{\stackrel{\infty}{q}}$ & & 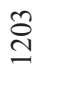 & ๙ิ & $\overrightarrow{\mathrm{N}}$ & $\stackrel{\infty}{\sim}$ & 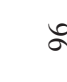 & & $\underline{6}$ & $\infty$ & के & $\stackrel{\infty}{\triangleright}$ & $\begin{array}{l}\infty \\
\infty \\
n\end{array}$ & ర్రి & in & $\underset{⿱}{\stackrel{f}{f}}$ & 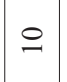 & $\cong$ & 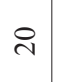 & ષ્તે \\
\hline$\overleftarrow{\check{\delta}}$ & 1 & O) & & 이 & O) & 1 & O & O) & & O & $\begin{array}{l}0 \\
1\end{array}$ & 足 & O & I & 이 & O & $\begin{array}{l}0 \\
\text { I }\end{array}$ & $\begin{array}{l}0 \\
1\end{array}$ & $\begin{array}{l}0 \\
1\end{array} \mid$ & 이 & 이 \\
\hline రั & ֻै & bे & 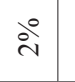 & غे & $\stackrel{\circ}{\circ}$ & $\ddot{\mathscr{O}}$ & $\stackrel{\stackrel{\circ}{\Xi}}{=}$ & $\stackrel{\circ}{0}$ & in & $\stackrel{\stackrel{\circ}{N}}{\stackrel{0}{0}}$ & $\frac{8}{i}$ & $\stackrel{\circ}{\circ}$ & 总 & 嗃 & in & $\stackrel{\stackrel{े}{ }}{ }$ & ஸें & $\stackrel{\circ}{\stackrel{0}{~}}$ & $\stackrel{\circ}{ٍ}$ & ठें & $\stackrel{\circ}{\infty}$ \\
\hline تै & 0 & $\begin{array}{l}\infty \\
0^{\circ}\end{array}$ & $\begin{array}{l}n \\
0^{\circ}\end{array}$ & $a$ & $\begin{array}{l}> \\
0\end{array}$ & $\begin{array}{l}\vec{z} \\
\vec{u} \\
0\end{array}$ & 0 & 0 & $\bar{z}$ & ט & $\begin{array}{l}\overrightarrow{\mid c} \\
0\end{array}$ & $\begin{array}{l}\overrightarrow{1} \\
\text { si }\end{array}$ & $\begin{array}{l}n \\
0 \\
0\end{array} \mid$ & 0 & $a$ & $a$ & $a$ & $\infty$ & $a$ & ن & $a$ \\
\hline 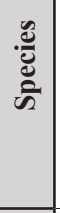 & & & & & & & & & & 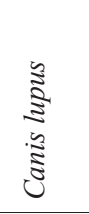 & & & & & & & & & & & \\
\hline$\sum_{i}$ & & & & & & & & & & שூ & & & & & & & & & & & \\
\hline \& & & & & & & & & & & 0 & & & & & & & & & & & \\
\hline
\end{tabular}




\begin{tabular}{|c|c|c|c|c|c|c|c|c|c|c|c|c|c|c|c|c|c|c|c|c|c|c|c|}
\hline 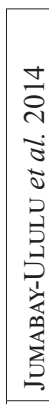 & 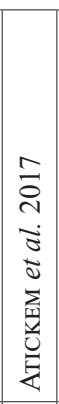 & 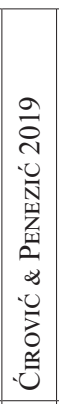 & 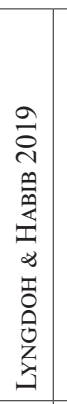 & 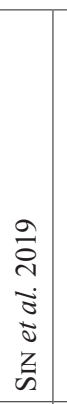 & 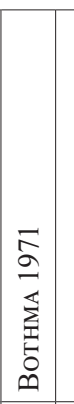 & 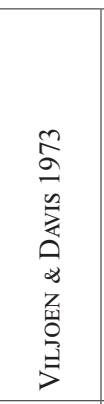 & 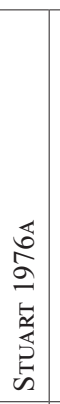 & 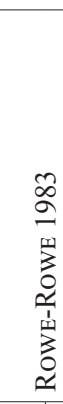 & & 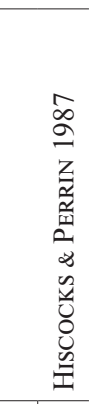 & & 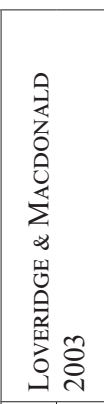 & 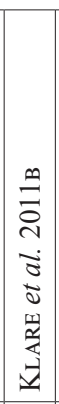 & 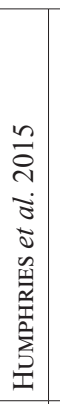 & 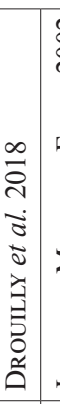 & 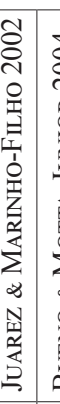 & 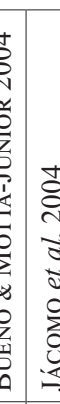 & & 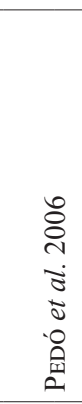 & & 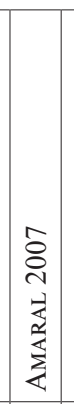 & 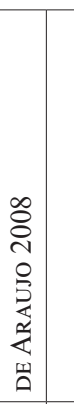 & 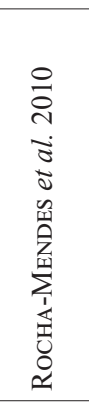 \\
\hline & 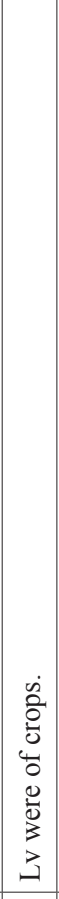 & 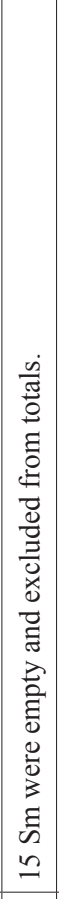 & 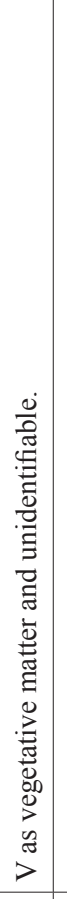 & 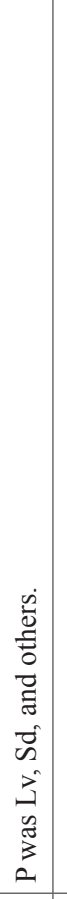 & 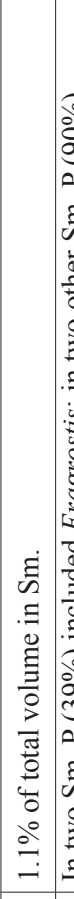 & 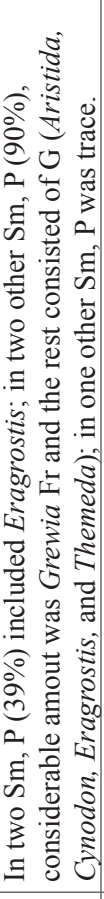 & & $\begin{array}{l}\dot{0} \\
0 \\
0 \\
0 \\
0 \\
0 \\
0 \\
3 \\
د\end{array}$ & & 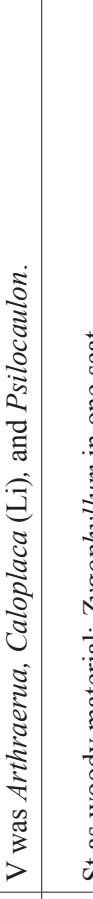 & 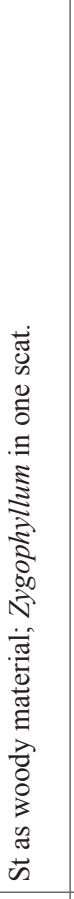 & & & & & & 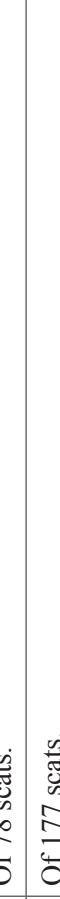 & & 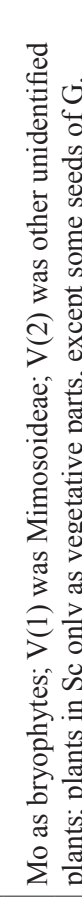 & & 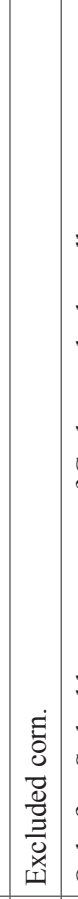 & 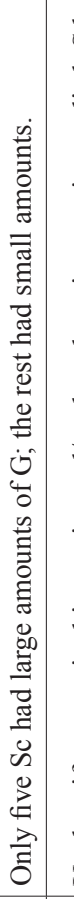 & 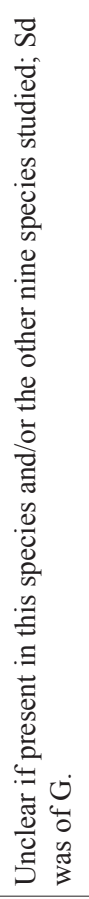 \\
\hline 识 & $\ddot{\mathscr{n}}$ & ڤ & $\ddot{\curvearrowleft}$ & $\ddot{n}$ & 苛 & हี & $\check{\curvearrowleft}$ & $\ddot{n}$ & $\dot{\varphi}$ & ố & $\ddot{n}$ & $\ddot{n}$ & $\ddot{\varkappa}$ & $\ddot{\curvearrowleft}$ & $\ddot{\mathscr{n}}$ & $\ddot{n}=$ & $\Rightarrow \pm$ & & $\ddot{n}$ & & 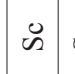 & $\ddot{n}$ & $\ddot{n}$ \\
\hline f & $\bar{\Xi}$ & 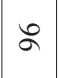 & $\stackrel{\infty}{=}$ & ণ & $\stackrel{\sim}{q}$ & in & 8 & 点 & $\stackrel{\overbrace{}}{\hat{f}}$ & ฮิ & f & के & 导 & 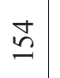 & $\bar{F}$ & ले & fे & & $\triangleright$ & & స్ & $\tilde{n}$ & + \\
\hline 1 & o & $\approx$ & 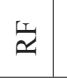 & O) & O) & $\vec{a}$ & 足 & 庄 & O) & o & o & O & O & 足 & o & o) & P & & 오 & & o & O & 1 \\
\hline खै & $\stackrel{\text { ڤे }}{\curvearrowright}$ & ¿ें & ळे & $\stackrel{\circ}{\circ}$ & dì & ڤें & @े & ஷे & $\frac{\circ}{n}$ & iें $\mathrm{i}^{\circ}$ & 80 & $\stackrel{\circ}{\grave{g}}$ & ठें & $\stackrel{\circ}{\beth}$ & ڤેं & 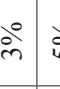 & $\stackrel{8}{\stackrel{0}{r}} \frac{\partial^{\circ}}{\mathrm{V}}$ & 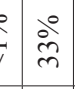 & ळे वें & 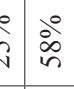 & iे & $\begin{array}{c}0 \\
\infty \\
\infty \\
\end{array}$ & $\ddot{\ddot{O}}$ \\
\hline$a$ & $\begin{array}{l}3 \\
0 \\
0\end{array}$ & $\begin{array}{l}3 \\
0 \\
0\end{array}$ & $>$ & $a$ & 0 & $a$ & ن & 3 & 0 & $>0$ & $\vec{\omega}$ & 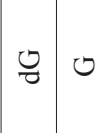 & $\begin{array}{l}3 \\
3 \\
0\end{array}$ & ن & $\begin{array}{l}3 \\
0 \\
0\end{array}$ & ט & ט & $\begin{array}{l}\Omega \\
0 \\
0\end{array}$ & $\stackrel{3}{2}$ & $E \underset{>}{\mathbb{d}}$ & O & 0 & $\begin{array}{l}\vec{\omega} \\
\dot{m} \\
\dot{0}\end{array}$ \\
\hline & & 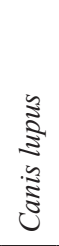 & & & & & & & & 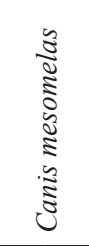 & & & & & & & & & 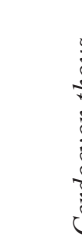 & 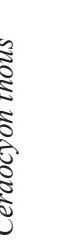 & & & \\
\hline & & & & & & & & & & & & $\pi$ & & & & & & & & & & & \\
\hline & & & & & & & & & & & & 0 & & & & & & & & & & & \\
\hline
\end{tabular}




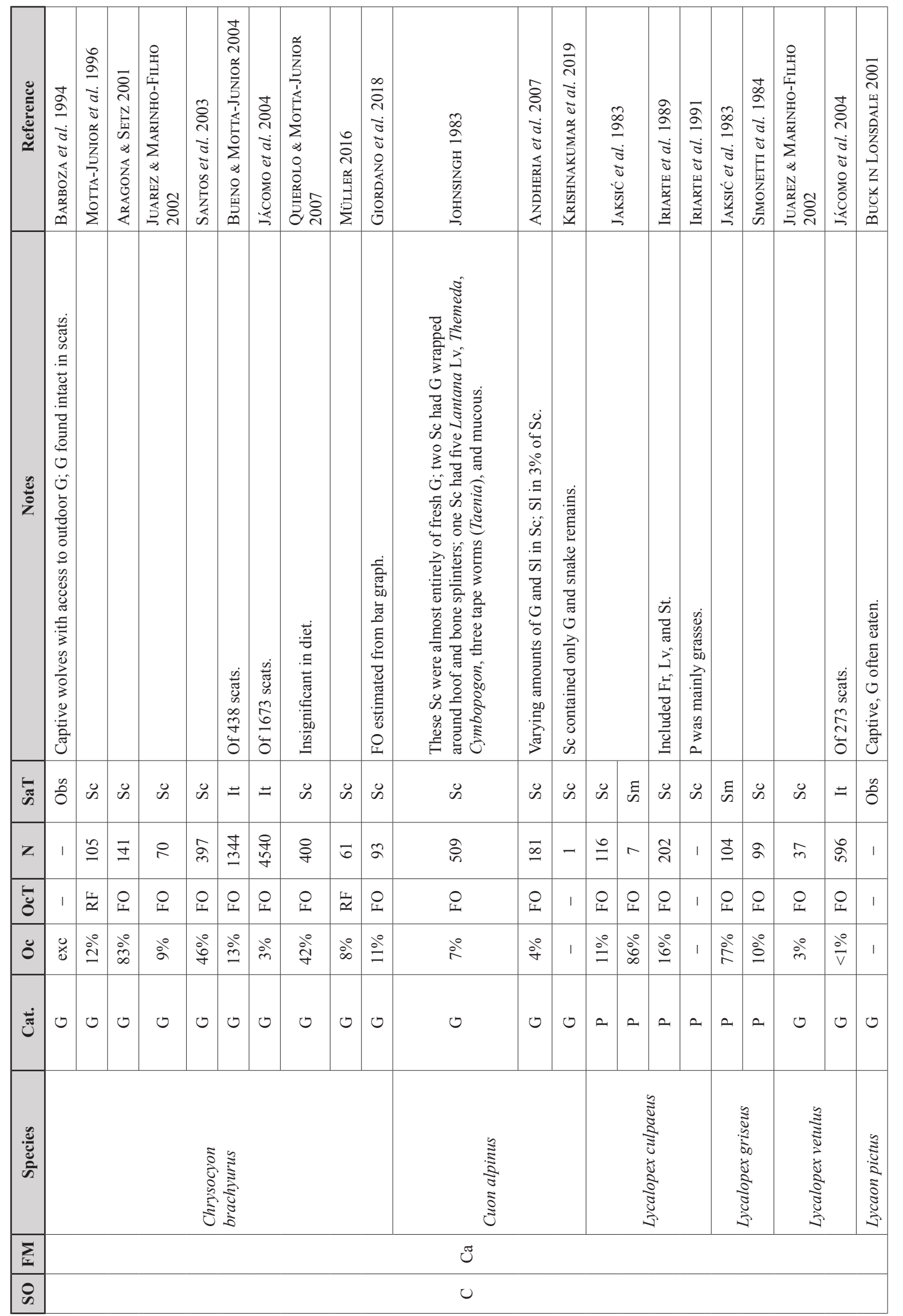




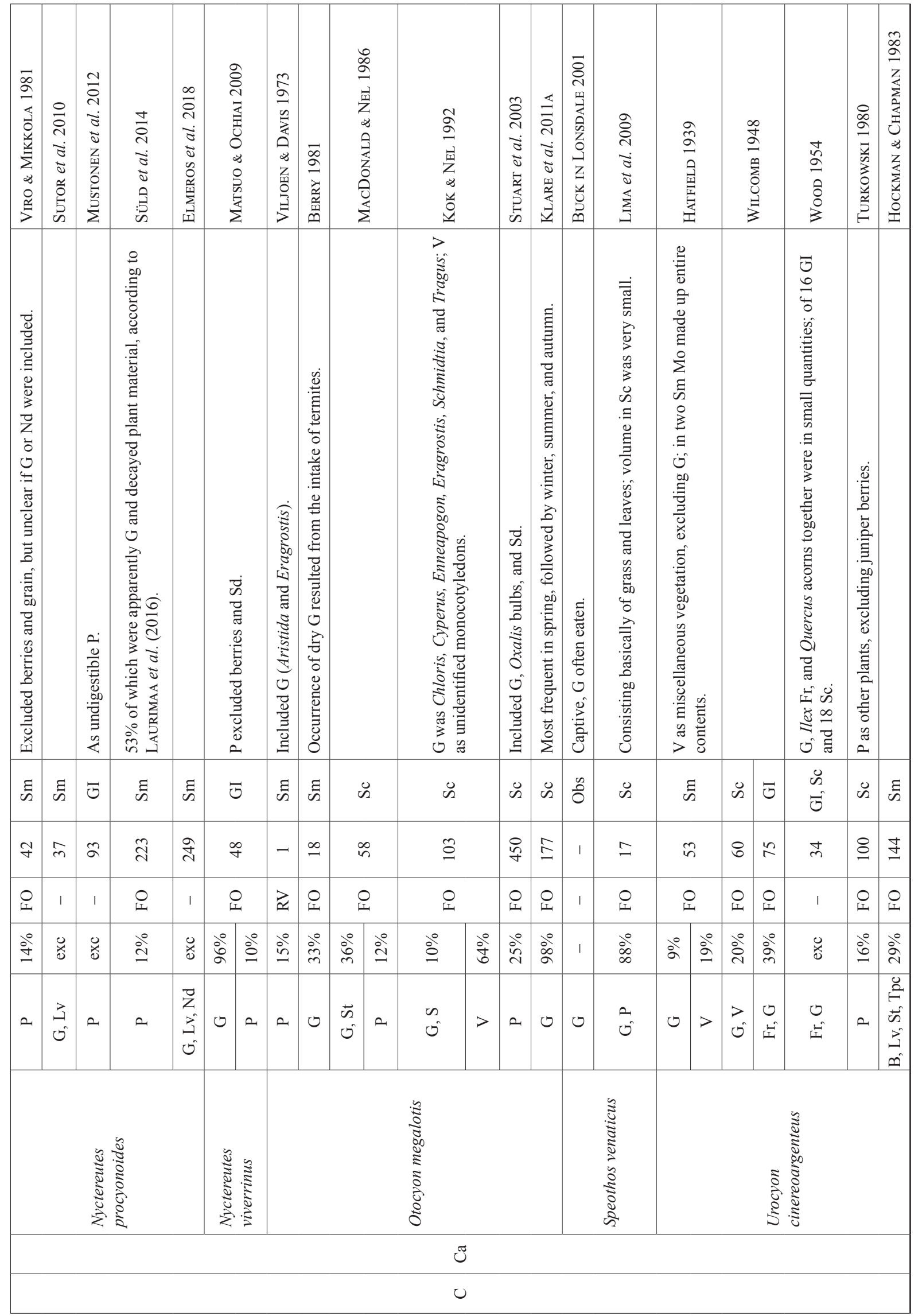




\begin{tabular}{|c|c|c|c|c|c|c|c|c|c|c|c|c|c|c|c|c|c|c|c|c|}
\hline 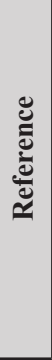 & 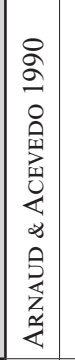 & 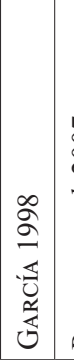 & 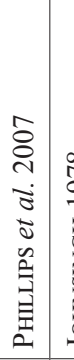 & 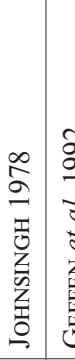 & 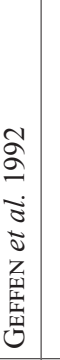 & 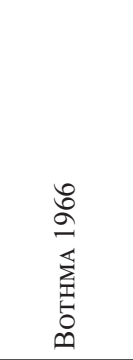 & 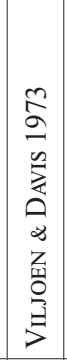 & 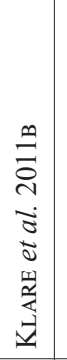 & 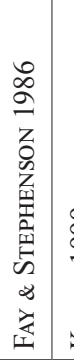 & 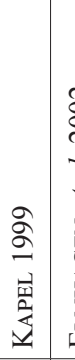 & 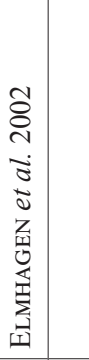 & 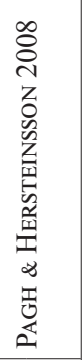 & 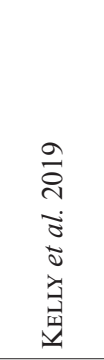 & 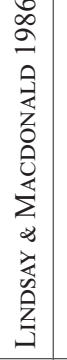 & 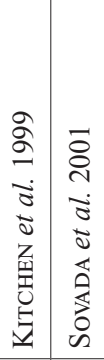 & 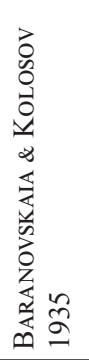 & 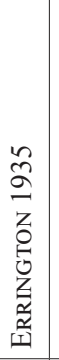 & 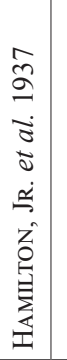 & 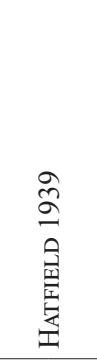 & 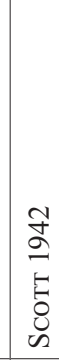 \\
\hline 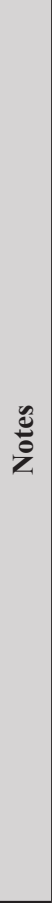 & & & & 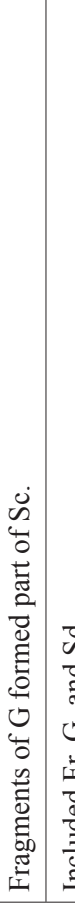 & 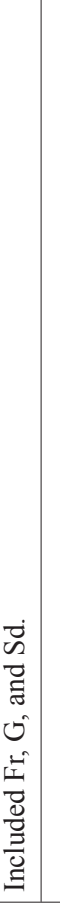 & 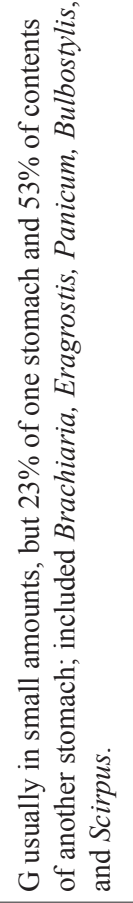 & & & 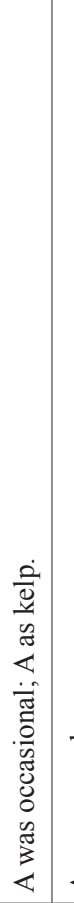 & 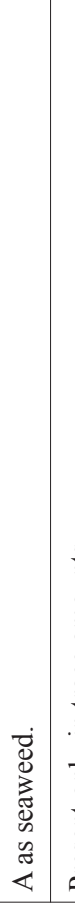 & 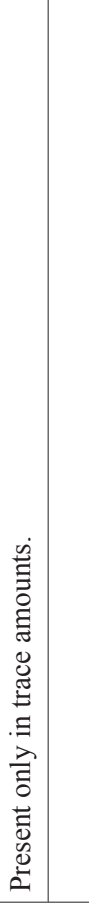 & 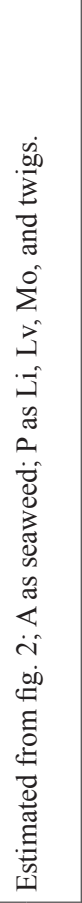 & 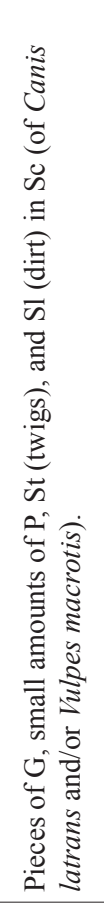 & 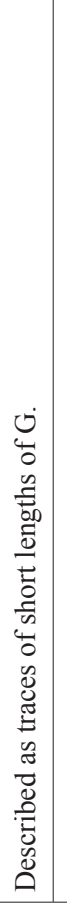 & 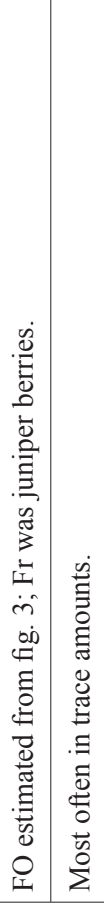 & 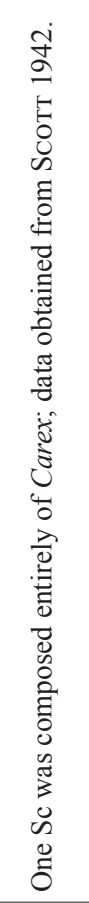 & 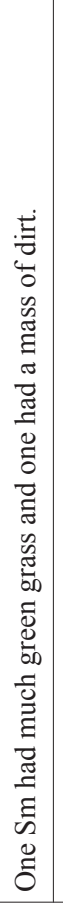 & 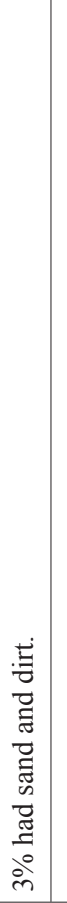 & 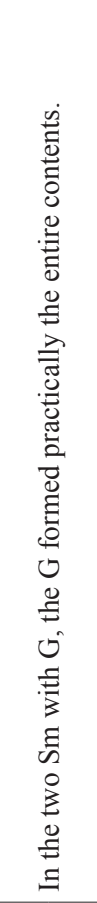 & 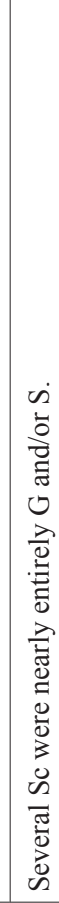 \\
\hline हू & $\ddot{n}$ & $\ddot{n}$ & $\ddot{n}$ & $\ddot{n}$ & $\ddot{n}$ & हี & Е & $\ddot{n}$ & घ & $\widetilde{J}$ & $\ddot{n}$ & ઘ & $\ddot{n}$ & $\ddot{n}$ & $\ddot{n} \ddot{n}$ & $\ddot{n}$ & ह & $\ddot{n}$ & ઘ & $\ddot{\mathscr{L}}$ \\
\hline z & $\vec{\Xi}$ & $\sim$ & $\stackrel{\infty}{\alpha}$ & 1 & 惫 & $\hat{m}$ & - & 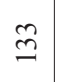 & $\stackrel{\infty}{\stackrel{\infty}{\beth}}$ & 売 & ते & $F$ & 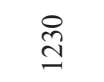 & $\nsubseteq$ & ڤิ) & 1 & in & $\hat{m}$ & ते & 蔮 \\
\hline $\bar{\delta}$ & 이 & $\Sigma$ & । & 1 & O & O & $\vec{z}$ & 이 & o & o & 이 & 오 & 1 & 이 & $\gtrsim$ O & 1 & 이 & o & 이 & 이 \\
\hline$\check{8}$ & $\stackrel{\circ}{ٍ}$ & $\mid \begin{array}{c}\infty 00 \\
\vec{i}\end{array}$ & $\ddot{\breve{o}}$ & 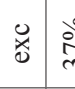 & 总 & $\frac{\partial^{\circ}}{n}$ & $\underset{\mathscr{E}}{\mathscr{E}}$ & 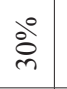 & $\ddot{\ddot{u}}$ & $\stackrel{\circ}{\circ}$ & 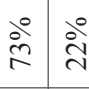 & $\begin{array}{l}\text { ì } \\
\text { vin }\end{array}$ & 1 & సें & ふे సे & । & $\stackrel{े}{\circ}$ & $\stackrel{\circ}{\vec{v}}$ & $\stackrel{\circ}{\stackrel{\circ}{+}}$ & $\stackrel{\circ}{\dot{\sigma}}$ \\
\hline Uี & ט & $a$ & $\begin{array}{l}\vec{n} \\
0\end{array}$ & 0 & a & $\begin{array}{l}n \\
0\end{array}$ & $a$ & $\begin{array}{l}3 \\
0 \\
0\end{array}$ & $\varangle$ & $\varangle$ & $>\ll$ & 40 & $\begin{array}{l}\vec{n} \\
\dot{c} \\
\dot{\theta}\end{array}$ & ט & \begin{tabular}{l|l}
$\vec{n}$ & \\
0 & 0 \\
$ن$ &
\end{tabular} & $\infty$ & ט & ט & 0 > & ט \\
\hline 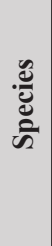 & $\begin{array}{r} \\
5 \\
5 \\
\overline{0} \\
5 \\
5\end{array}$ & 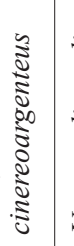 & 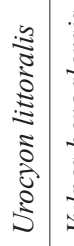 & 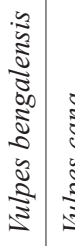 & 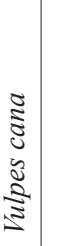 & 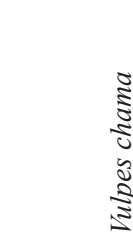 & & & & & 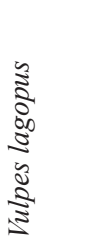 & & 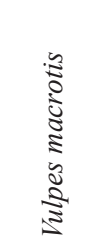 & 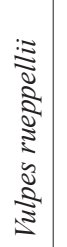 & 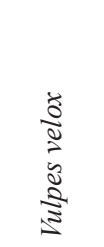 & & & 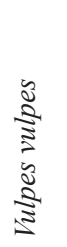 & & \\
\hline$\sum_{\Sigma}$ & \multicolumn{20}{|c|}{$\pi$} \\
\hline o & & & & & & & & & & & u & u & & & & & & & & \\
\hline
\end{tabular}




\begin{tabular}{|c|c|c|c|c|c|c|c|c|c|c|c|c|c|c|c|c|c|c|c|c|c|c|c|}
\hline 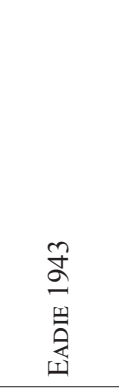 & 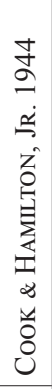 & $\begin{array}{l}\frac{\infty}{0} \\
\frac{1}{3} \\
0 \\
0 \\
0\end{array}$ & & 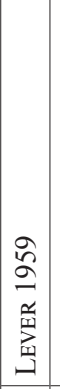 & 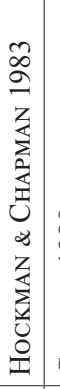 & 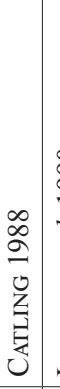 & 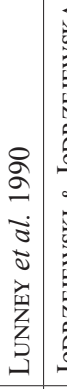 & 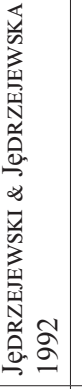 & 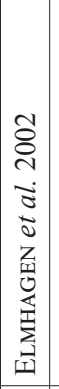 & 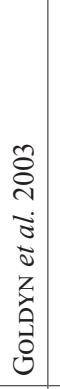 & 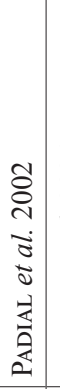 & 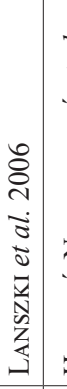 & 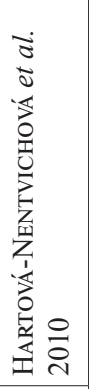 & 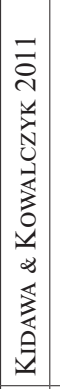 & 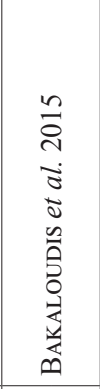 & \multicolumn{5}{|c|}{ 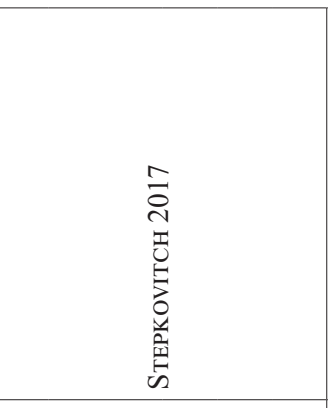 } & 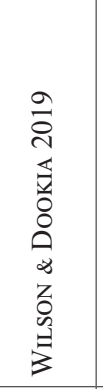 & 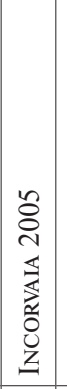 & 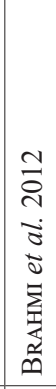 \\
\hline 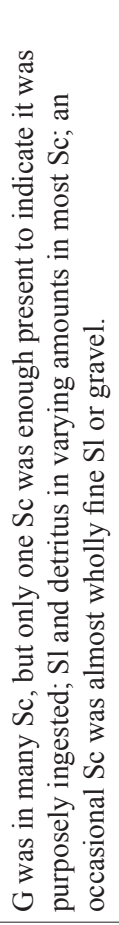 & 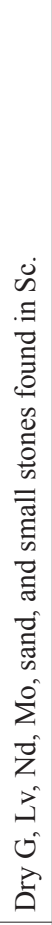 & & & 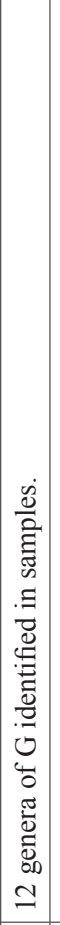 & & 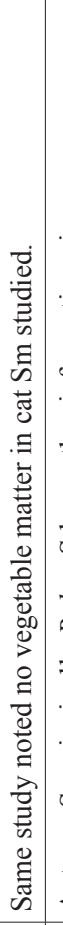 & 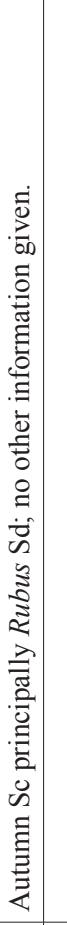 & 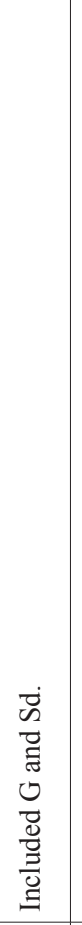 & 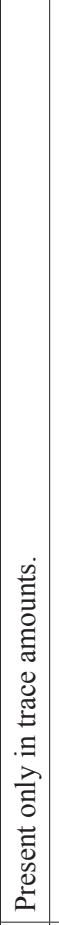 & 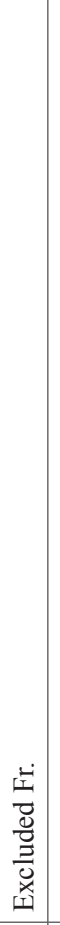 & & 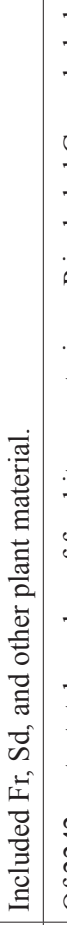 & 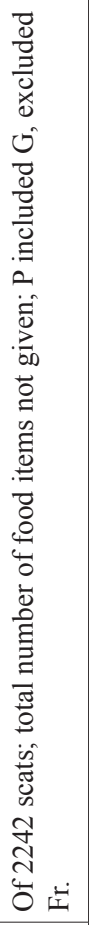 & & 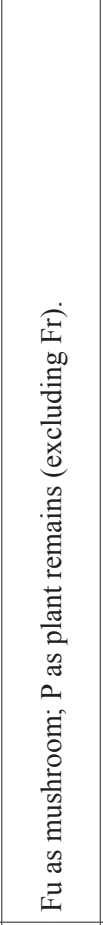 & \multicolumn{5}{|c|}{ 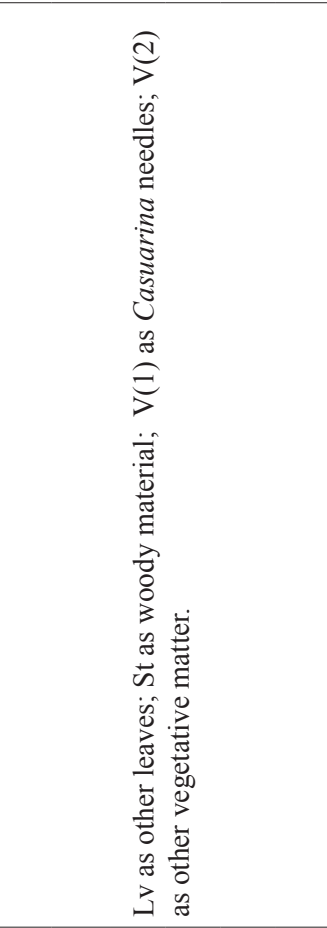 } & 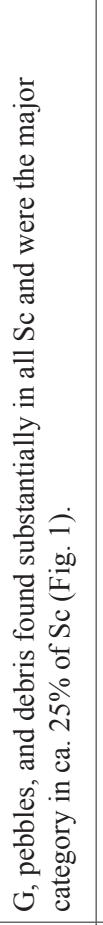 & 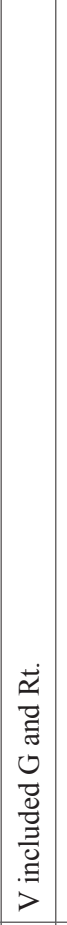 & 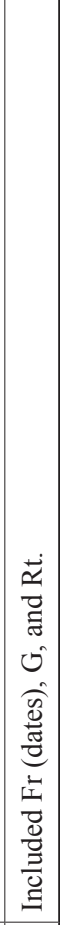 \\
\hline$\ddot{n}$ & $\ddot{n}$ & $\ddot{n}$ & $\vec{J}$ & $\begin{array}{l}E \\
w \\
\dot{n} \\
\dot{n}\end{array}$ & ह & ह & $\ddot{n}$ & $\ddot{n}$ & $\ddot{\curvearrowleft}$ & $\ddot{n}$ & $\ddot{n}$ & $\ddot{n}$ & $=$ & 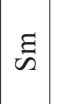 & हี & \multicolumn{5}{|c|}{ घ } & $\ddot{n}$ & $\ddot{\curvearrowleft}$ & $\ddot{n}$ \\
\hline$\frac{m}{m}$ & $\hat{n}$ & $\stackrel{8}{-1}$ & q & 畄 & 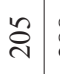 & $\begin{array}{l}\infty \\
\stackrel{\infty}{\sim} \\
\sim\end{array}$ & $\frac{m}{6}$ & $\underset{\sim}{\stackrel{్}{ల}}$ & $\cong$ & 응 & ֻั & $\stackrel{+}{\infty}$ & a. & $\stackrel{ \pm}{\sim}$ & $\stackrel{\vec{\lambda}}{\vec{\lambda}}$ & \multicolumn{5}{|c|}{ 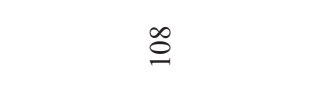 } & 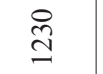 & $\vec{\sim}$ & $\stackrel{\leftrightarrow}{\varrho}$ \\
\hline O & I & o & $\begin{array}{l}0 \\
\end{array}$ & 1 & O & O & O & O) & O & 足 & O & $\sum$ & O & $\begin{array}{l}0 \\
\text { I }\end{array}$ & O & \multicolumn{5}{|c|}{ O } & 1 & $\stackrel{\square}{\simeq}$ & O \\
\hline$\frac{\stackrel{\circ}{\vec{v}}}{\mathrm{v}}$ & ע્̋ & 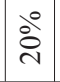 & 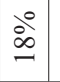 & $\ddot{凶}$ & ڤें & ஷें & ஓें & $\stackrel{\circ}{=}$ & ì & ठें & $\stackrel{\circ}{\Rightarrow}$ & $\begin{array}{l}0 \\
\infty \\
\infty\end{array}$ & $\stackrel{\circ}{=}$ & $\mid \begin{array}{c}0 \\
\dot{0} \\
\sim \\
\sim\end{array}$ & 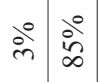 & $\stackrel{\circ}{\dot{\sigma}}$ & ठें & خें & के & $\stackrel{\circ}{\circ}$ & 1 & ذें & iें \\
\hline 0 & $\begin{array}{l}\frac{0}{0} \\
ن\end{array}$ & $\begin{array}{l}> \\
0\end{array}$ & $\left|\begin{array}{c}0 \\
ث \\
1 \neq\end{array}\right|$ & 0 & $\begin{array}{l}\vec{n} \\
\vec{\Delta} \\
\vec{n} \\
\boldsymbol{n}^{\prime}\end{array}$ & 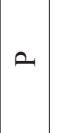 & a & $a$ & $>$ & $\approx$ & 0 & a & $a$ & 0 & $\overrightarrow{\vec{x}} a$ & $\vec{I}$ & 0 & $\vec{\omega}$ & $\stackrel{Ð}{\supset}$ & $\widehat{d}$ & $\begin{array}{l}\dot{0} \\
0 \\
0\end{array}$ & $>$ & $a$ \\
\hline & & & & & & & & & & & & 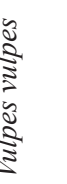 & & & & & & & & & & 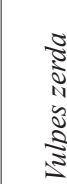 & 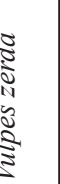 \\
\hline \multicolumn{24}{|c|}{$\pi$} \\
\hline & & & & & & & & & & & & & u & & & & & & & & & & \\
\hline
\end{tabular}




\begin{tabular}{|c|c|c|c|c|c|c|c|c|c|c|c|c|c|c|c|c|c|c|c|c|}
\hline 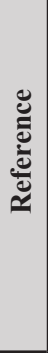 & 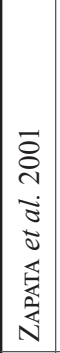 & 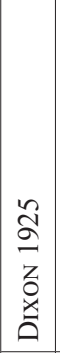 & 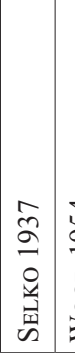 & 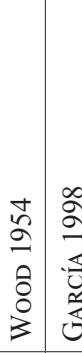 & 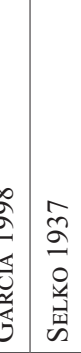 & 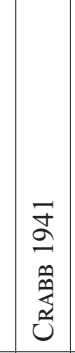 & 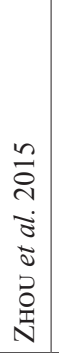 & 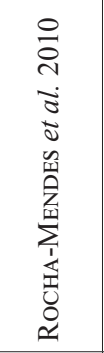 & 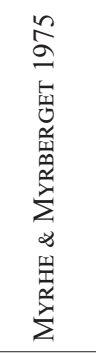 & 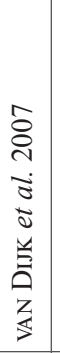 & 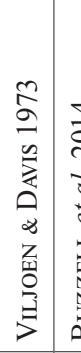 & 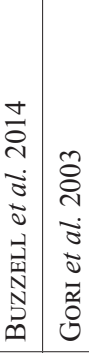 & 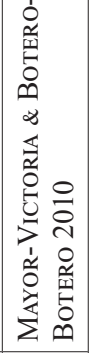 & 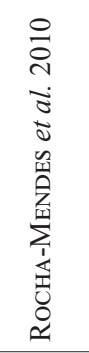 & 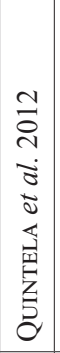 & 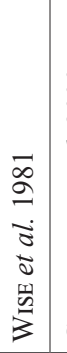 & 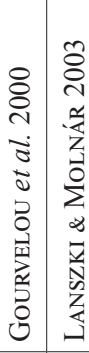 & 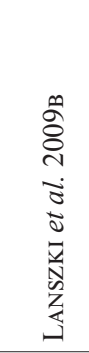 & 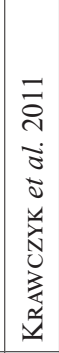 & 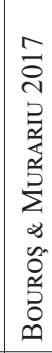 \\
\hline $\begin{array}{l}\text { है } \\
\text { z }\end{array}$ & 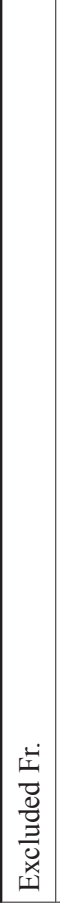 & & 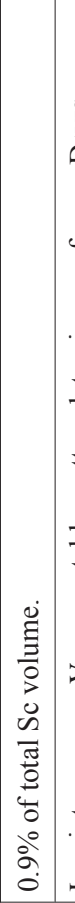 & 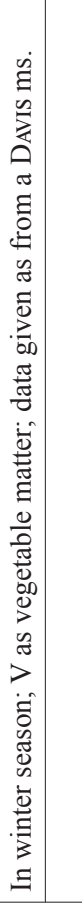 & 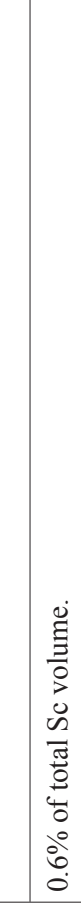 & 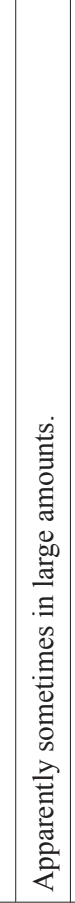 & & 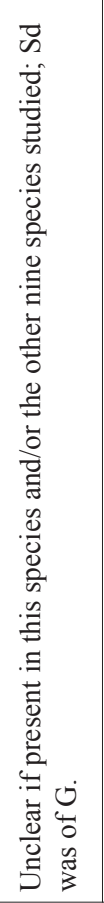 & 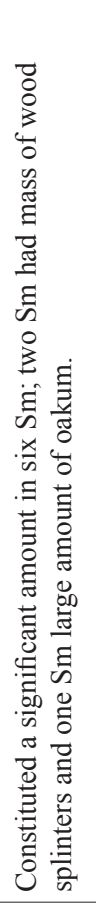 & 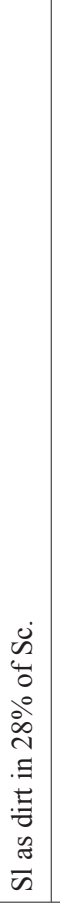 & & & & 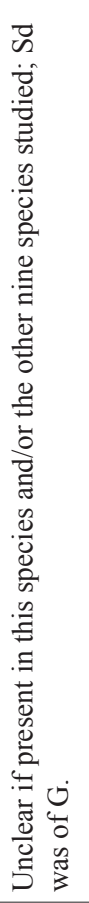 & $\begin{array}{l}\dot{0} \\
\overline{0} \\
\frac{d}{0} \\
\frac{\Xi}{0} \\
\stackrel{\Xi}{>} \\
>\end{array}$ & 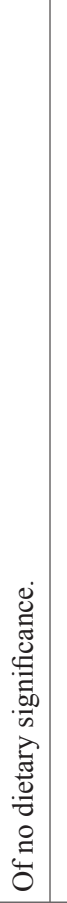 & 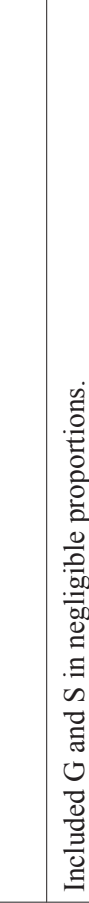 & 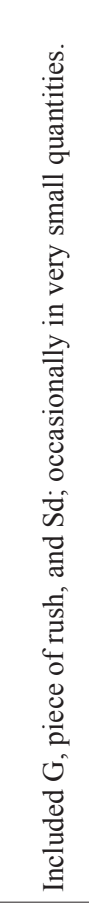 & 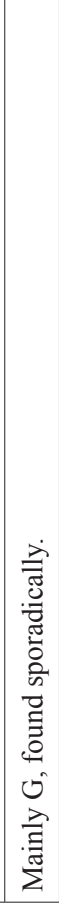 & 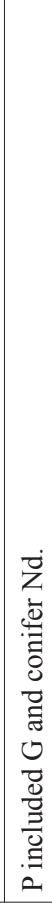 \\
\hline$\overline{\tilde{n}}$ & $\ddot{n}$ & $\Xi$ & $\ddot{n}$ & $1 \ddot{n}$ & $\begin{array}{l}u \\
n\end{array}$ & $\ddot{n}$ & $\ddot{n}$ & $\ddot{n}$ & घ & $\ddot{n}$ & ह & $\check{\check{n}} \check{\mathscr{n}}$ & $\ddot{n}$ & $\ddot{n}$ & $\ddot{n}$ & $\ddot{n}$ & $\ddot{\mathscr{n}} \ddot{\mathscr{n}}$ & $\ddot{n}$ & $\ddot{n}$ & $\ddot{n}$ \\
\hline z & $\overline{\vec{\lambda}}$ & $\ddot{m}$ & 守 & $1-$ & - in & $\begin{array}{l}\mathbb{J} \\
\infty\end{array}$ & 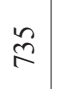 & - & $\bar{\Xi}$ & 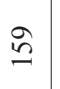 & - & $\stackrel{\vec{\nu}}{\stackrel{\tilde{d}}{c}}$ & $\vec{\sigma}$ & \pm & $\ddot{m}$ & 苦 & $\bar{n} \bar{n}$ & $\stackrel{8}{8}$ & ते & $\stackrel{ \pm}{I}$ \\
\hline $\bar{\delta}$ & o & o & $\begin{array}{ll}0 \\
1\end{array}$ & $\gtrsim$ & $\sum 0$ & o & 이 & 1 & 오 & o & $\vec{\sim}$ & \begin{tabular}{l|l} 
\\
\end{tabular} & $\stackrel{5}{2}$ & 1 & O & $\sum_{\Perp}$ & 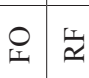 & 1 & 1 & O \\
\hline ठ & $\therefore$ & $\begin{array}{c}0 \\
\infty \\
\stackrel{े}{N}\end{array}$ & 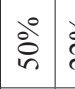 & 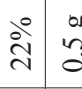 & \begin{tabular}{l|l}
0 \\
co
\end{tabular} & $\stackrel{\circ}{\forall}$ & $\stackrel{\circ}{\infty}$ & ֶै. & iें & 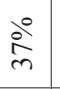 & $\stackrel{\circ}{\circ}$ & ¿ें & $\stackrel{\circ}{\dot{\sigma}}$ & $\ddot{凶}$ & $\frac{\partial^{\circ}}{\vec{v}}$ & $\stackrel{\circ}{\vec{v}}$ & $\stackrel{\circ}{\circ} \stackrel{\circ}{\circ}$ & $\ddot{x}$ & $\ddot{\mho}$ & $\stackrel{\circ}{\circ}$ \\
\hline تี & $a$ & $\mid \begin{array}{l}\vec{\approx} \\
0\end{array}$ & $>$ & $>$ & $>$ & ט & $\begin{array}{l}\vec{n} \\
\vec{\sim} \\
\vec{\Delta} \\
\overrightarrow{0} \\
\dot{0}\end{array}$ & $\begin{array}{l}\vec{n} \\
\dot{n} \\
\dot{0}\end{array}$ & $>$ & $a$ & $a$ & $\begin{array}{ll}2 & 0\end{array}$ & $>$ & $\begin{array}{l}\vec{n} \\
\dot{n} \\
\dot{0}\end{array}$ & $>$ & $a$ & $a$ a & $a$ & $a$ & $a$ \\
\hline 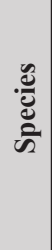 & 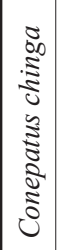 & & 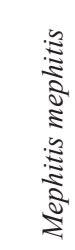 & 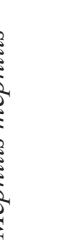 & & 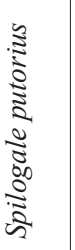 & 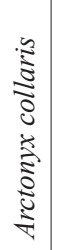 & 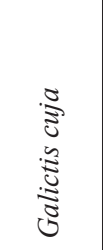 & 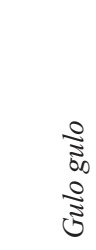 & & 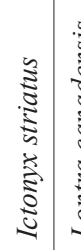 & 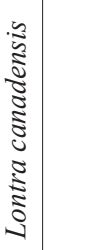 & & & & & & 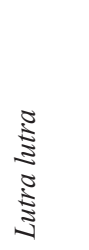 & & \\
\hline$\sum_{\Sigma}$ & \multicolumn{6}{|c|}{$\sum$} & \multicolumn{14}{|c|}{$\Sigma$} \\
\hline O & & & & & & & & & & & 0 & & & & & & & & & \\
\hline
\end{tabular}




\begin{tabular}{|c|c|c|c|c|c|c|c|c|c|c|c|c|c|c|c|c|c|c|c|c|c|}
\hline 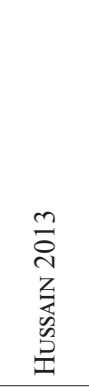 & $\begin{array}{l}5 \\
0 \\
0\end{array}$ & & $\begin{array}{l}\tilde{n} \\
\sigma \\
\underline{0} \\
\tilde{a} \\
a\end{array}$ & 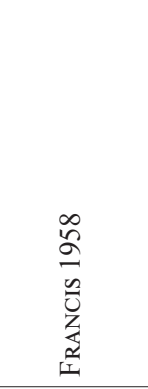 & 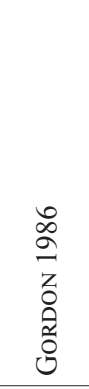 & 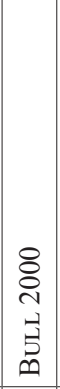 & 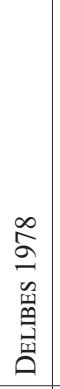 & 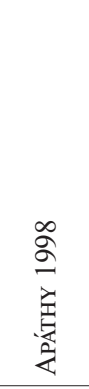 & 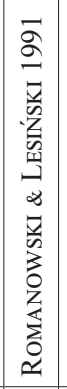 & 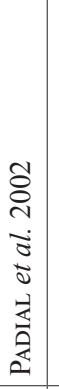 & 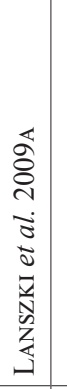 & 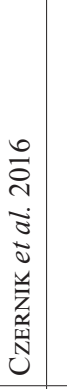 & 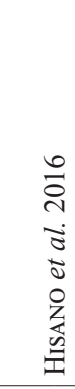 & & 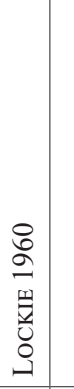 & 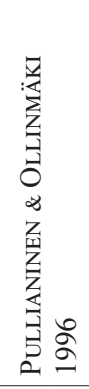 & 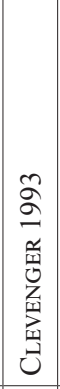 & 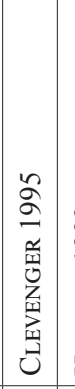 & 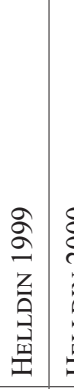 & 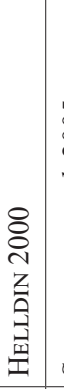 & 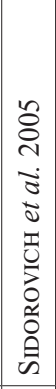 \\
\hline 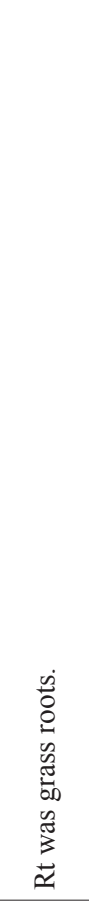 & 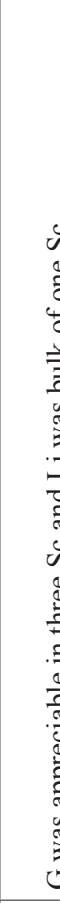 & 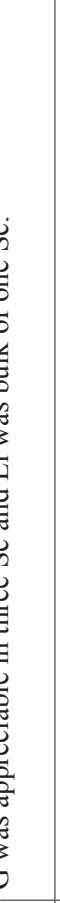 & & 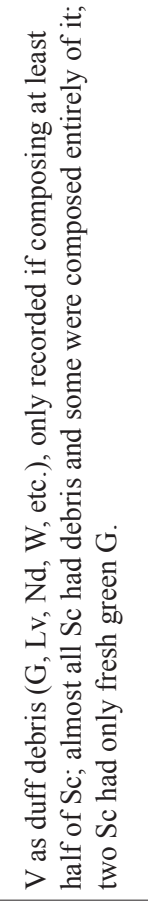 & 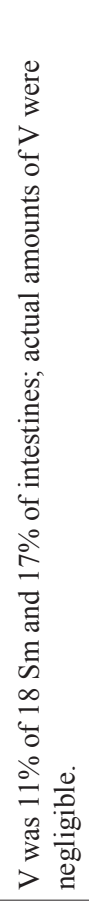 & 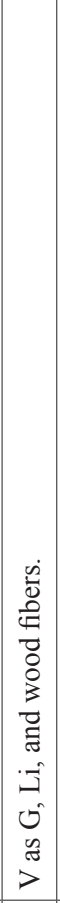 & 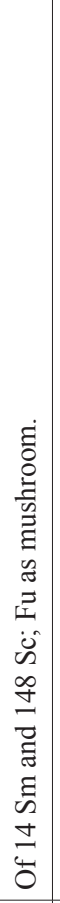 & 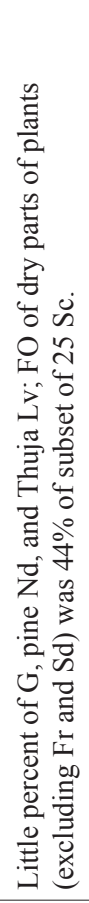 & 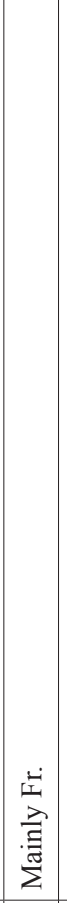 & & 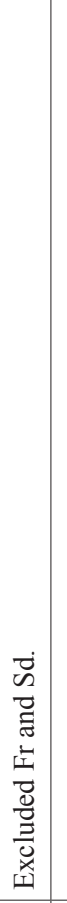 & 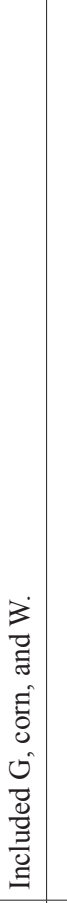 & 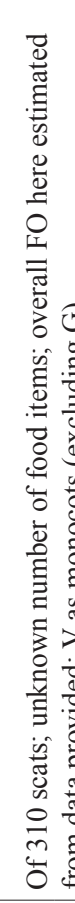 & 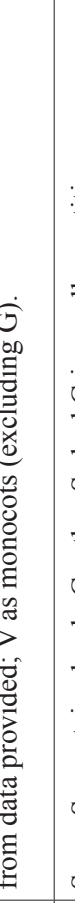 & 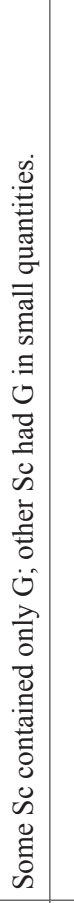 & 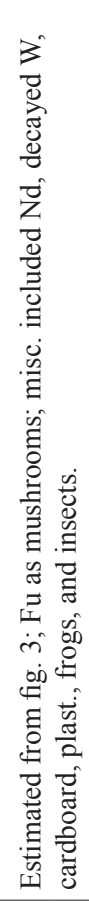 & & & 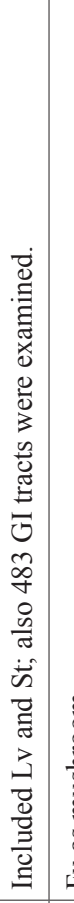 & 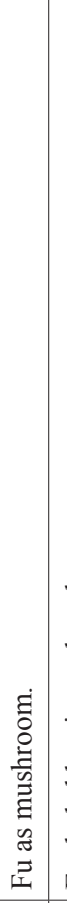 & 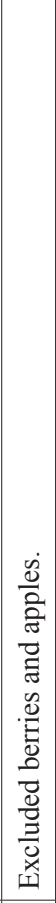 \\
\hline$\ddot{n}$ & v & 号 & $\ddot{n}$ & \pm & $\overparen{D}$ & $\ddot{n}$ & $\begin{array}{l}घ \\
\tilde{n} \\
\dot{n}\end{array}$ & $\ddot{n}$ & $\ddot{\curvearrowleft}$ & $\ddot{n}$ & $\ddot{n}$ & $\ddot{n}$ & \pm & & $\ddot{n}$ & $\ddot{n}$ & $\ddot{n}$ & $\ddot{n}$ & 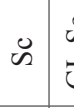 & $\begin{array}{l}\dot{n} \\
\tilde{n}\end{array}$ & $\ddot{n}$ \\
\hline$\tilde{n}$ & 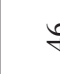 & o & $\begin{array}{l}\stackrel{2}{n} \\
\end{array}$ & $\widetilde{\Omega}$ & $\approx$ & $\stackrel{\Xi}{\Xi}$ & $\hat{n}$ & $\infty$ & $\hat{\varrho}$ & 8 & $\widehat{\mathbb{I}}$ & $\widehat{\widetilde{\infty}}$ & 1 & & $\bar{m}$ & $\stackrel{\hat{0}}{0}$ & $\stackrel{m}{m}$ & $\stackrel{\infty}{\mathbb{N}}$ & $\stackrel{\infty}{\stackrel{m}{~}}$ & 号 & ปี \\
\hline O & 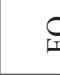 & t & O) & 이 & 이 & o & Oે & 1 & o & O & o & O & 이 & o & $\sum$ & $\vec{\approx}$ & $\begin{array}{l}0 \\
0\end{array}$ & 이 & 1 & o & O \\
\hline ڤे & $\stackrel{\circ}{\circ}$ & $\stackrel{\circ}{\mathrm{i}}$ & $\frac{\partial^{\circ}}{\vec{v}}$ & के & $=\stackrel{\circ}{\stackrel{0}{g}}$ & in & $\frac{\partial^{\circ}}{\vec{v}}$ & 艹ै & ¿ें & $\frac{\partial}{\vec{v}}$ & @े & iे & $\frac{\partial}{\vec{v}}$ & 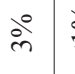 & $\frac{\partial}{\vec{v}}$ & $\frac{\stackrel{\circ}{\vec{v}}}{\stackrel{\circ}{+}}$ & $\stackrel{े}{\mathrm{i}}$ & $\frac{\partial}{\vec{v}}$ & 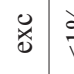 & $\stackrel{\stackrel{\circ}{v}}{\vec{v}}$ & $\frac{\partial}{\vec{v}}$ \\
\hline $\begin{array}{l}\vec{\approx} \\
\overrightarrow{0}\end{array}$ & ט & $\exists$ & ט & $>$ & $>$ & $>$ & $\overrightarrow{\mid \vec{x}}$ & $a$ & $a$ & ט & 2 & a & ט & $>$ & ט. & \begin{tabular}{l|l}
$\dot{v}$ & $\dot{m}$ \\
\end{tabular} & 0 & 0 & $>$ & 至 & 0 \\
\hline 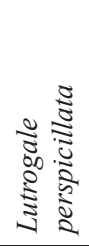 & & & & 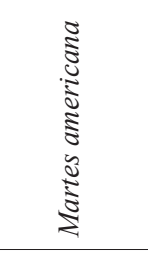 & & & \multicolumn{8}{|c|}{ 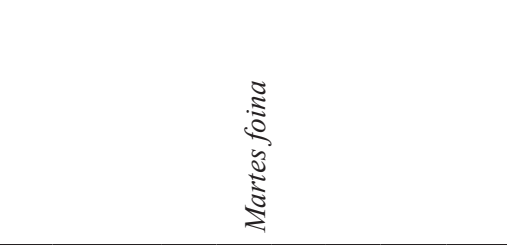 } & \multicolumn{7}{|c|}{ 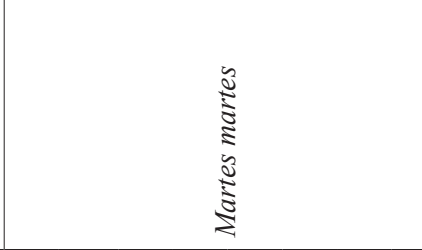 } \\
\hline \multicolumn{22}{|c|}{$\sum_{\Sigma}^{E}$} \\
\hline & & & & & & & & & U & & & & & & & & & & & & \\
\hline
\end{tabular}




\begin{tabular}{|c|c|c|c|c|c|c|c|c|c|c|c|c|c|c|c|c|c|c|c|}
\hline 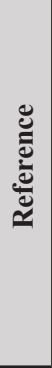 & 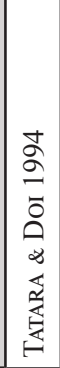 & 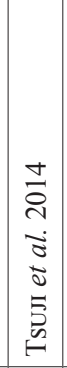 & 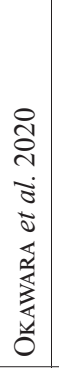 & 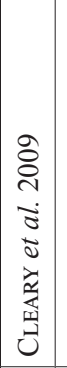 & 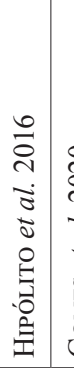 & 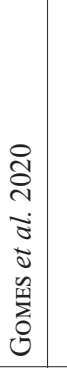 & 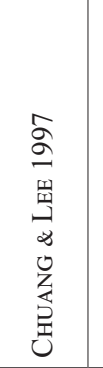 & 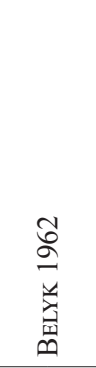 & 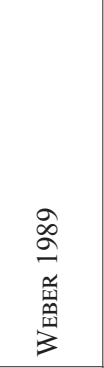 & 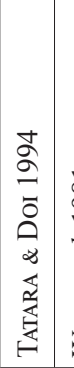 & 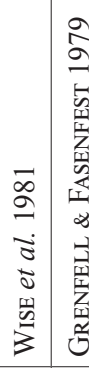 & 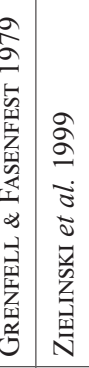 & & 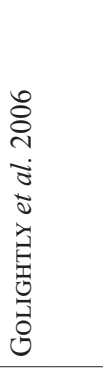 & 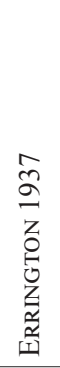 & 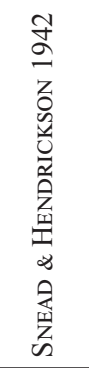 & & 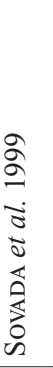 & \\
\hline : & 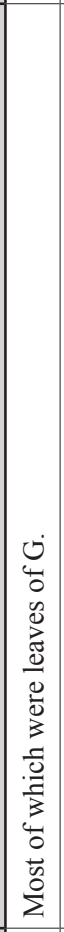 & 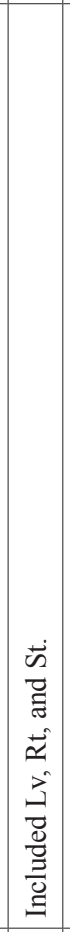 & & 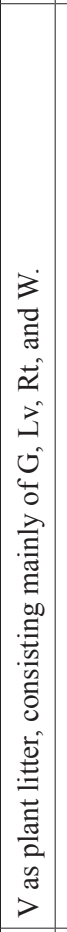 & 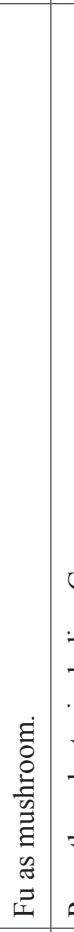 & 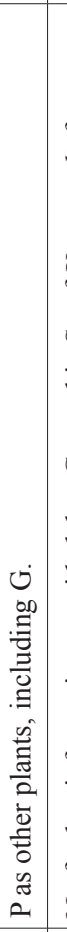 & 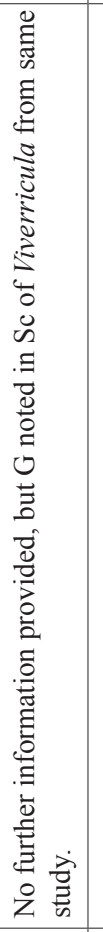 & 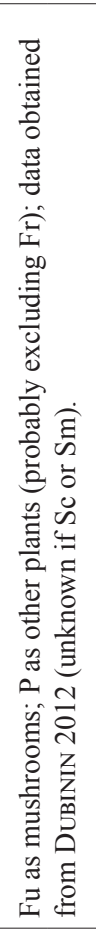 & 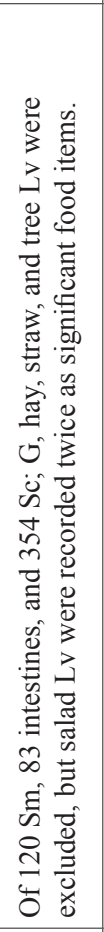 & 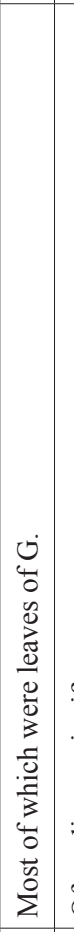 & 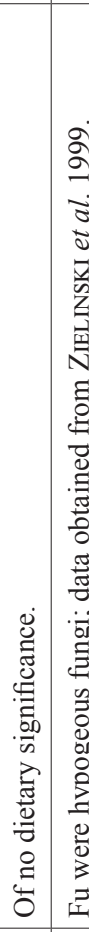 & 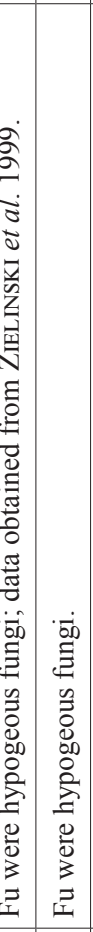 & & 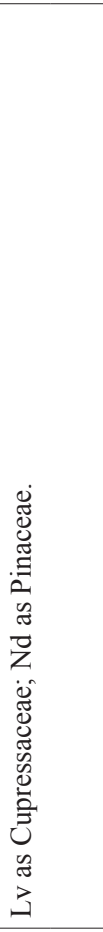 & 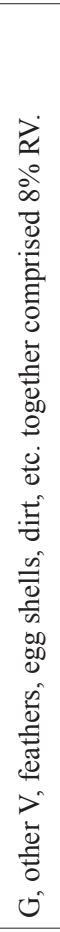 & 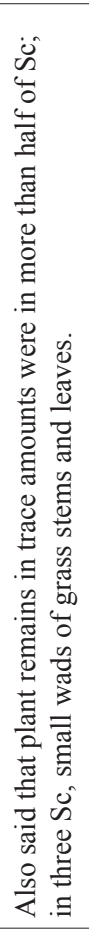 & & 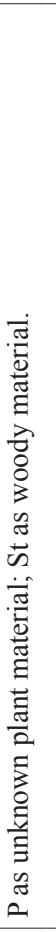 & \\
\hline हू & 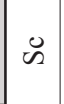 & $\ddot{\curvearrowleft}$ & $\ddot{n}$ & E & $\ddot{\infty}$ & ह & $\ddot{n}$ & 1 & $\begin{array}{l}\ddot{\leftrightarrow} \\
\tilde{0}\end{array}$ & $\ddot{n}$ & $\ddot{\varkappa}$ क & $\ddot{n}$ & & $\ddot{n}$ & $\ddot{n}$ & $\ddot{n}$ & & $\widetilde{\sigma}$ & \\
\hline z & $\underset{\widetilde{\beth}}{\beth}$ & $\stackrel{\sim}{\Im}$ & $\stackrel{\circ}{\stackrel{\sim}{\sim}}$ & $\stackrel{0}{0}$ & $\frac{\infty}{m}$ & के & 5 & $\underset{\mathscr{\alpha}}{\mathscr{\alpha}}$ & 1 & $\stackrel{\infty}{\sim}$ & $\frac{m}{n} \infty$ & $\infty \quad \frac{A}{4}$ & & $\underset{\infty}{\infty}$ & $\hat{m}$ & $\stackrel{\infty}{\infty}$ & & in & \\
\hline$\overline{\check{o}}$ & o & O & 1 & o & 오 & 이 & 이 & 오 & 1 & O & $\sum_{\Omega 1} 0$ & \begin{tabular}{l|l}
0 & 0 \\
&
\end{tabular} & & 이 & $\gtrsim$ & 이 & & 오 & \\
\hline ธ & $\stackrel{\circ}{\circ}$ & ळें & 1 & ळें & iें & ìं & 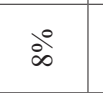 & iे iे & $\ddot{x}$ & $\stackrel{े}{\circ}$ & $\stackrel{\partial}{\vec{v}} \stackrel{\circ}{\dot{\nu}^{\circ}}$ & $\begin{array}{l}0 \\
\stackrel{\circ}{n}\end{array}$ & bें & ذి & 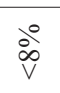 & $\stackrel{\circ}{\varrho}$ & 守 & iे & ڤे \\
\hline تี & $a$ & $a$ & 3 & $>$ & 山 & $a$ & $a$ & 妵a & $a$ & $a$ & $a$ 至 & $\overrightarrow{\vec{\tau}} \mid \vec{\nabla}$ & ט & $\vec{z} \bar{z}$ & 0 & $a$ & ن & $a$ & $\ddot{\omega}$ \\
\hline 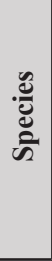 & & 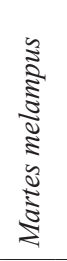 & & & 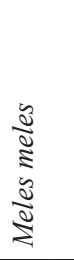 & & 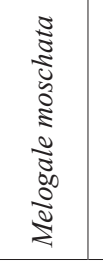 & 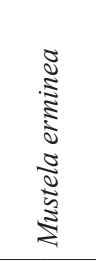 & 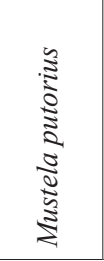 & 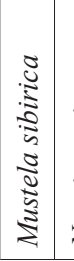 & 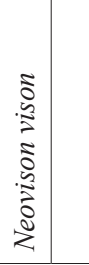 & & 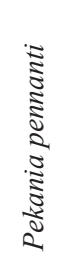 & & & 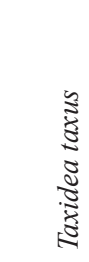 & & & \\
\hline$\sum_{\Sigma}$ & \multicolumn{19}{|c|}{$\sum_{\Sigma}$} \\
\hline \& & & & & & & & & & & & 0 & & & & & & & & \\
\hline
\end{tabular}




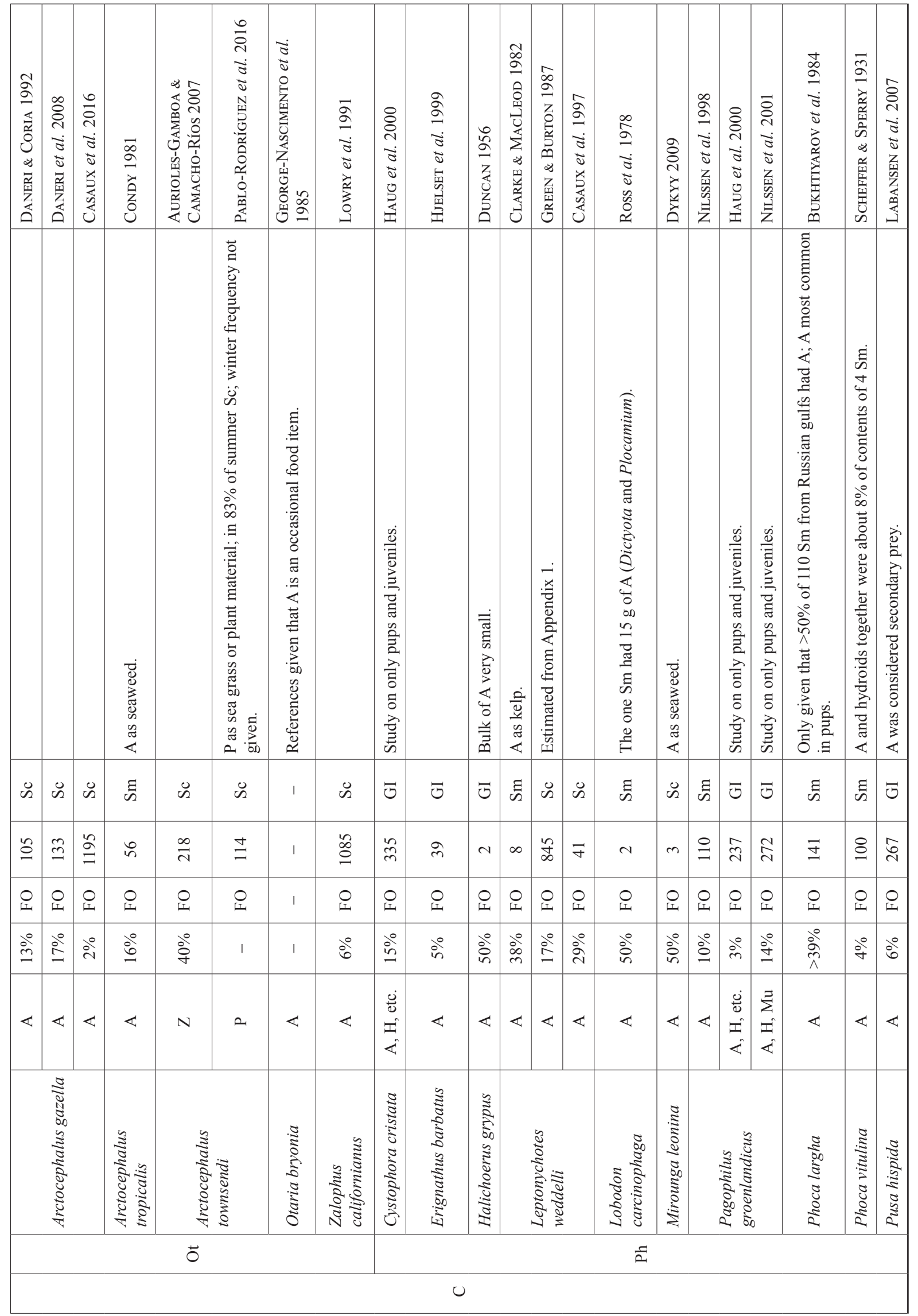




\begin{tabular}{|c|c|c|c|c|c|c|c|c|c|c|c|c|c|c|c|c|c|c|c|c|}
\hline$\stackrel{\square}{\check{L}}$ & 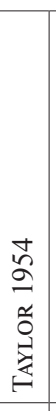 & 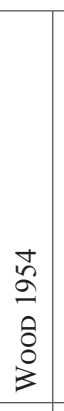 & 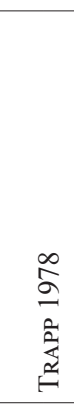 & & & & & 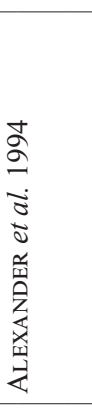 & & & & 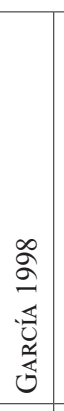 & 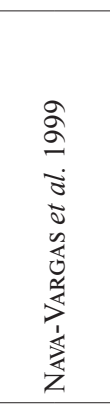 & 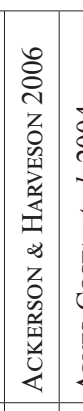 & 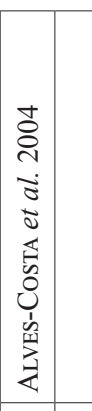 & 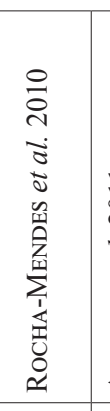 & 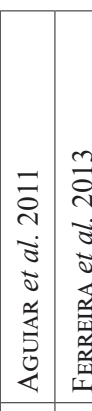 & 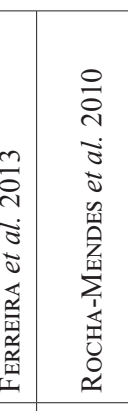 & 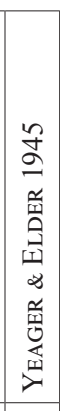 & 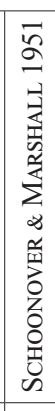 \\
\hline 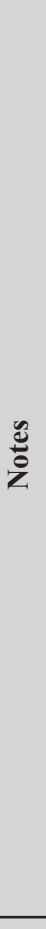 & 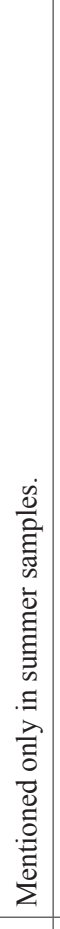 & 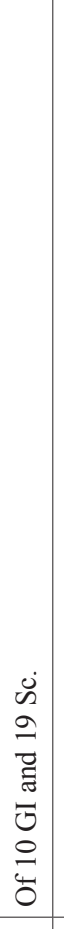 & 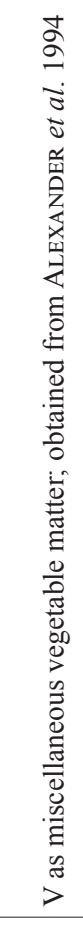 & & & & 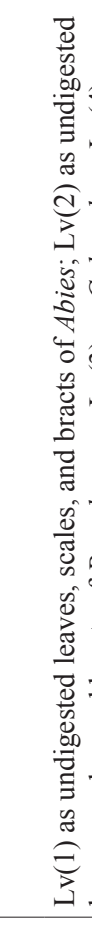 & 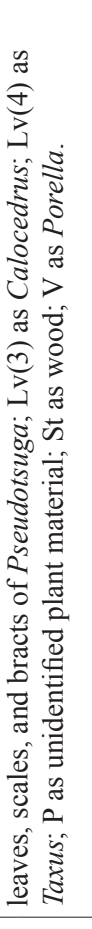 & & & & & & 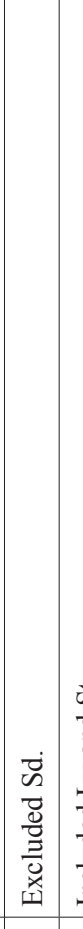 & 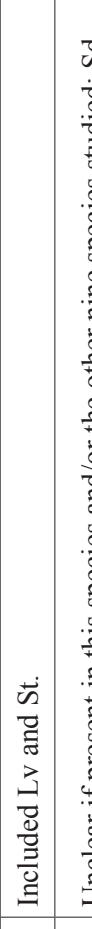 & 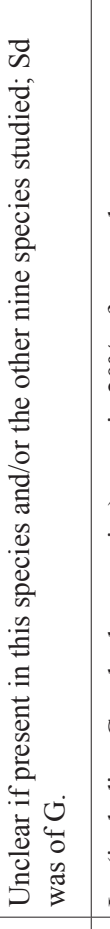 & 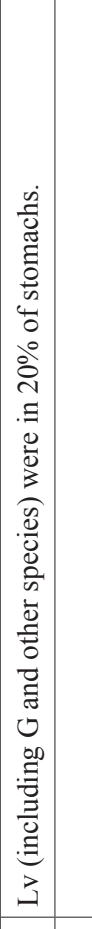 & 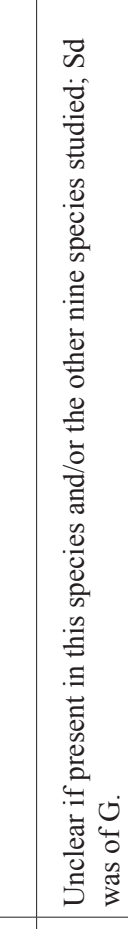 & 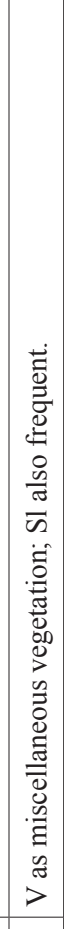 & \\
\hline है & उ) & $\begin{array}{l}\dot{0} \\
\tilde{u} \\
\tilde{0}\end{array}$ & $\ddot{n}$ & & & & & $\ddot{n}$ & & & & $\ddot{~}$ & $\ddot{n}$ & $\begin{array}{lll}\dot{m} & 0\end{array}$ & $\ddot{n}$ & $\ddot{n}$ & 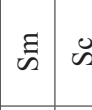 & $\ddot{n}$ & $\ddot{n}$ & $\ddot{8}$ \\
\hline $\mathrm{z}$ & : & ते & 1 & & & & & $i n$ & & & & $m$ & 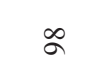 & 8 & : & $\stackrel{\infty}{\infty}$ & $\approx \sim$ & - & สิ & 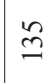 \\
\hline $\bar{\Xi}$ & $\stackrel{ \pm}{\approx}$ & $\vec{a}$ & 임 & & & & & 이 & & & & $\Sigma$ & 이 & o & 오 & 1 & 인 & 1 & 있 & 오 \\
\hline 8 & $\frac{\overrightarrow{\vec{v}}}{\mathrm{v}}$ & $\stackrel{\mathscr{E}}{\mathscr{E}}$ & ชั ปे & in & $\stackrel{\stackrel{\circ}{ \pm}}{\dot{0}}$ & ัें & ذ્ల్ల & $\stackrel{\circ}{\sim}$ & ப் & $\stackrel{\circ}{=}$ & $\stackrel{\circ}{\circ}$ & 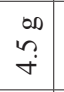 & $\stackrel{\circ}{\mathrm{C}} \stackrel{\mathrm{\partial}}{\mathrm{m}}$ & 总 & \begin{tabular}{|c|} 
\\
$\vdots$ \\
$\infty$ \\
$\infty$
\end{tabular} & & $\stackrel{\circ}{\circ} \stackrel{\circ}{\circ}$ & $\stackrel{\mathscr{\%}}{\%}$ & ڤें & $\frac{\dot{V}^{\circ}}{\mathrm{V}}$ \\
\hline ฮี & 0 & 0 & $0>$ & 0 & ت & (ְ) & 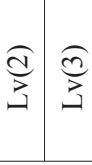 & 悉 & $\stackrel{\circ}{\Sigma}=$ & $\vec{i}$ & $>$ & 3 & II 3 & $>$ & $>$ & $\begin{array}{l}\vec{v} \\
\vec{s} \\
s \\
0\end{array}$ & $0>$ & $\begin{array}{l}\vec{n} \\
\dot{n} \\
\dot{0}\end{array}$ & $>$ & 0 \\
\hline بू. & & & & & & & & 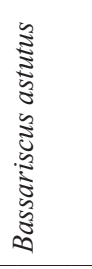 & & & & & & & & 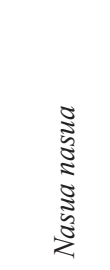 & & 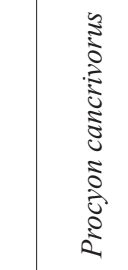 & & \\
\hline$\sum$ & & & & & & & & & & & \pm & & & & & & & & & \\
\hline \& & & & & & & & & & & & 0 & & & & & & & & & \\
\hline
\end{tabular}




\begin{tabular}{|c|c|c|c|c|c|c|c|c|c|c|c|c|c|c|c|c|c|c|c|}
\hline $\begin{array}{l}\text { पे } \\
\text { aे } \\
\overrightarrow{\hat{o}} \\
\vdots \\
\vdots\end{array}$ & 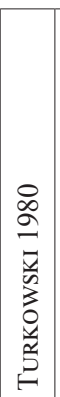 & 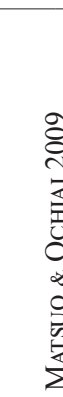 & & 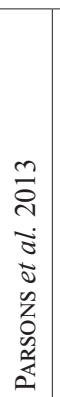 & 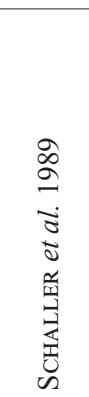 & 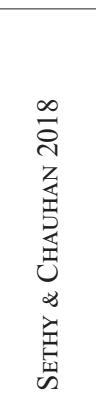 & 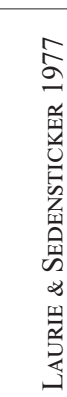 & & 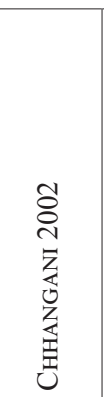 & 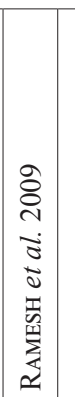 & 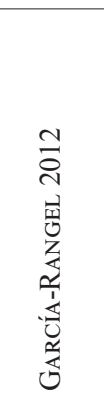 & 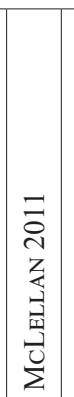 & 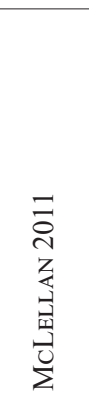 & 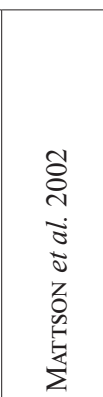 & 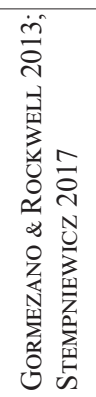 & 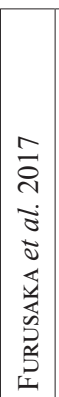 & 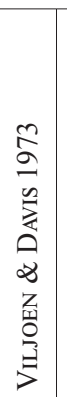 & 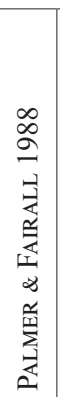 & 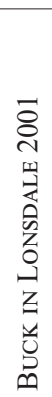 \\
\hline 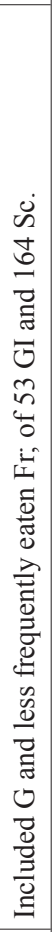 & & 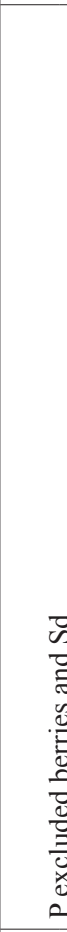 & & 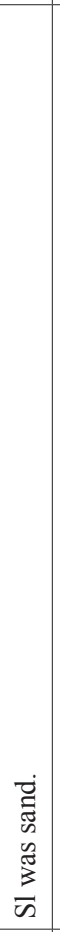 & 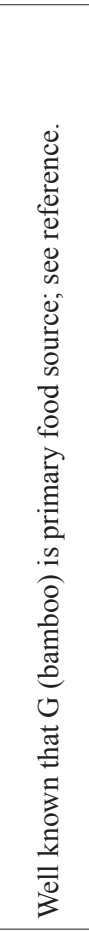 & 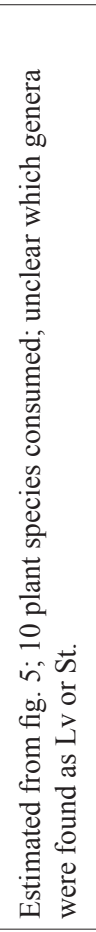 & 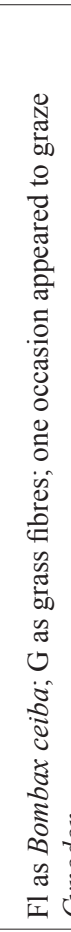 & & 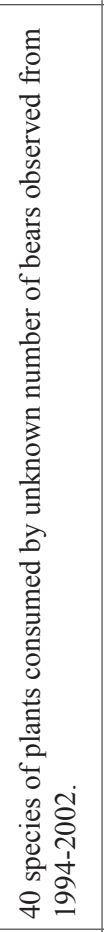 & 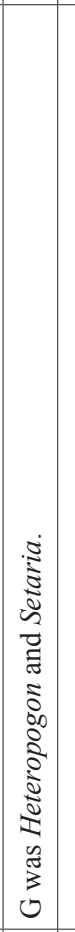 & 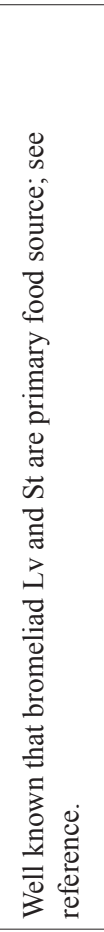 & 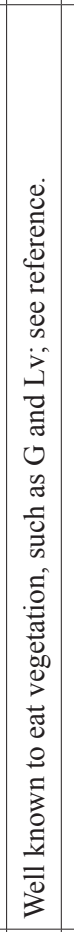 & 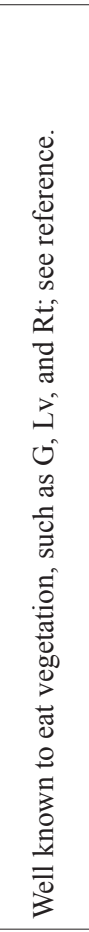 & 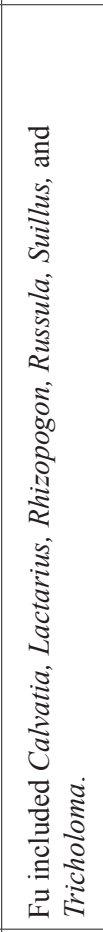 & 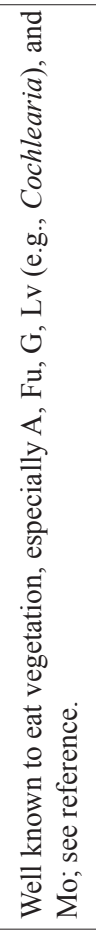 & 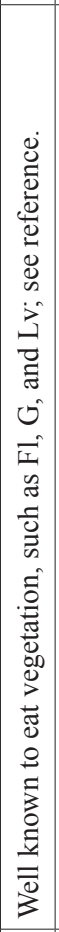 & & 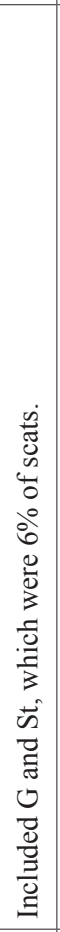 & 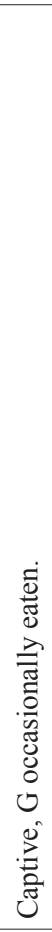 \\
\hline $\begin{array}{l}\dot{n} \\
\tilde{u}\end{array}$ & $\ddot{n}$ & $\tilde{U}$ & & ह & 1 & & $\ddot{n}$ & & 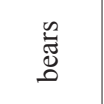 & $\ddot{n}$ & 1 & 1 & 1 & $\ddot{n}$ & 1 & 1 & ह & $\ddot{n}$ & $\stackrel{\infty}{0}$ \\
\hline$\overline{\vec{\nu}}$ & $\stackrel{\circ}{\circ}$ & ఠ̊ & & ஜ & 1 & & ले & & 1 & $\tilde{\sigma}$ & 1 & 1 & 1 & $\stackrel{8}{\frac{8}{r}}$ & 1 & 1 & $\sim$ & 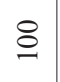 & 1 \\
\hline$\vec{a}$ & O & 오 & & $\sum_{\approx}$ & 1 & $\frac{1}{\simeq}$ & 이 & & I & O & 1 & 1 & 1 & 이 & 1 & 1 & $\vec{\imath}$ & 이 & 1 \\
\hline$\stackrel{\circ}{\stackrel{\circ}{v}}$ & $\stackrel{\circ}{\circ}$ & 总 & ळे & ठิे & । & 宂 & $\underset{\mathscr{E}}{\mathscr{E}}$ & $\stackrel{\circ}{\circ}$ & 1 & iे & 1 & 1 & 1 & $\stackrel{\circ}{\circ}$ & 1 & 1 & $\stackrel{\circ}{\infty}$ & $\stackrel{\circ}{\unrhd}$ & 1 \\
\hline $\begin{array}{l}0 \\
0\end{array}$ & 0 & 0 & $a$ & $\begin{array}{l}3 \\
\vec{n} \\
\dot{\tilde{n}} \\
\dot{0}\end{array}$ & ט & $\begin{array}{l}\vec{n} \\
\vec{n} \\
\overrightarrow{3} \\
\vec{y}\end{array}$ & $\vec{I}$ & 0 & 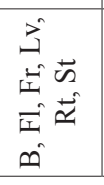 & 0 & $\breve{n}$ & $a$ & $a$ & $\overrightarrow{\mid \overrightarrow{\mid}}$ & $a$ & $a$ & $a$ & $>$ & 0 \\
\hline & & 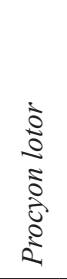 & & & 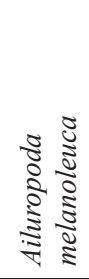 & 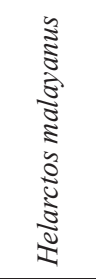 & & 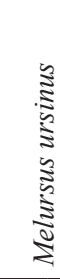 & & & 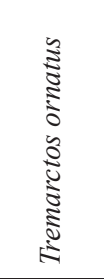 & 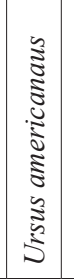 & & & 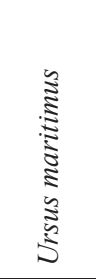 & 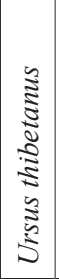 & & $\begin{array}{l}\bar{\Xi} \\
\bar{\Xi} \\
\bar{\Xi} \\
\bar{\Xi} \\
\bar{\Xi} \\
\end{array}$ & \\
\hline \multicolumn{5}{|c|}{ 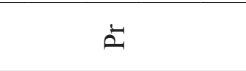 } & \multicolumn{12}{|c|}{5} & \multicolumn{3}{|c|}{ 車 } \\
\hline \multicolumn{17}{|c|}{ U } & & 山 & \\
\hline
\end{tabular}




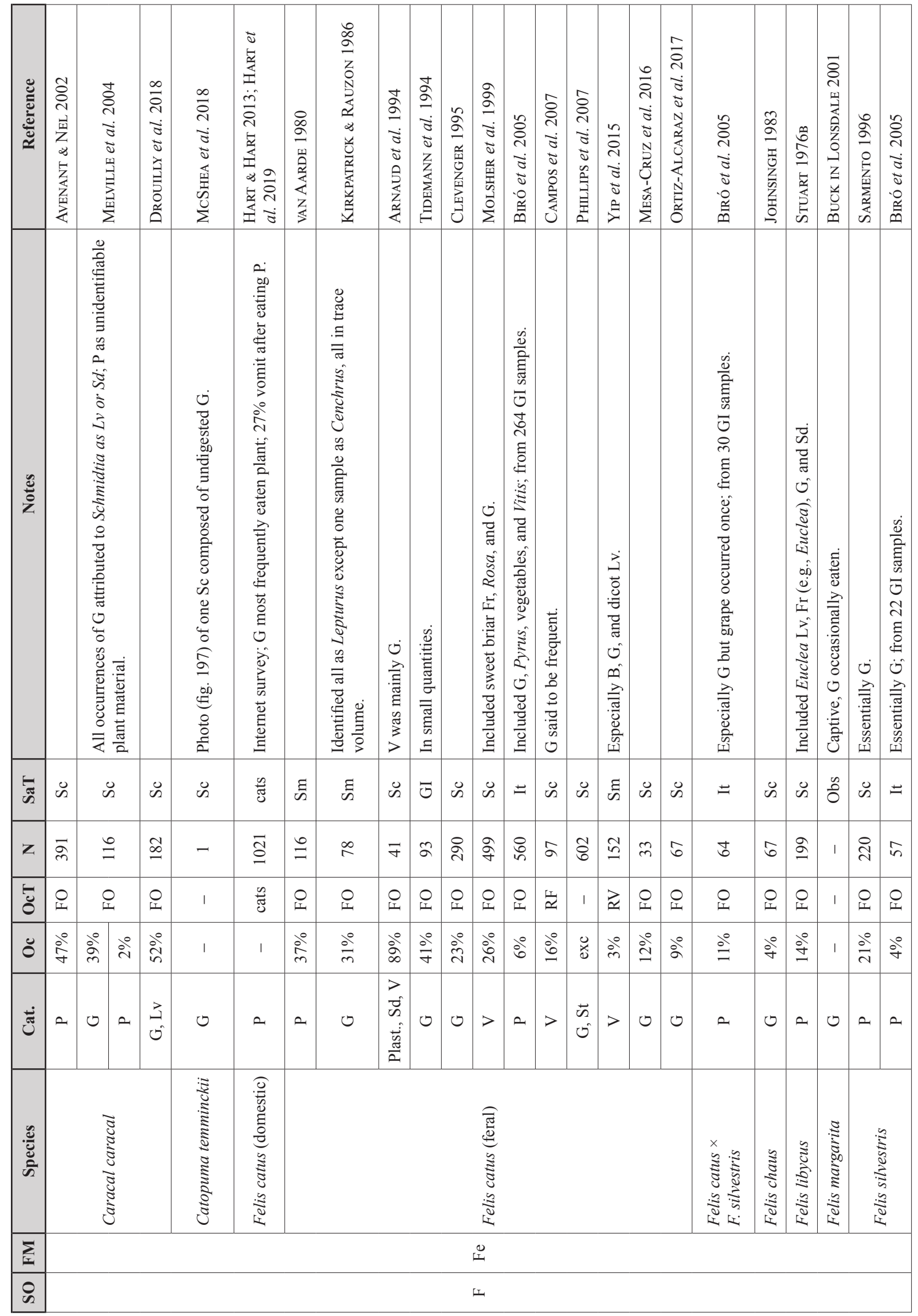




\begin{tabular}{|c|c|c|c|c|c|c|c|c|c|c|c|c|c|c|c|c|c|c|c|c|}
\hline 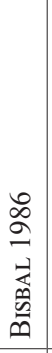 & 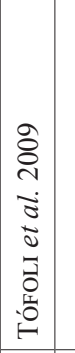 & 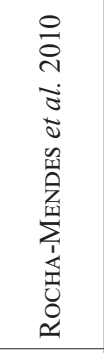 & 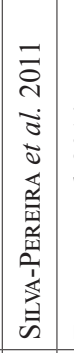 & 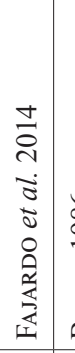 & 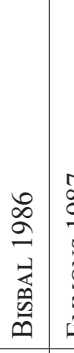 & 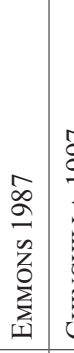 & 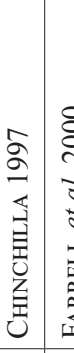 & 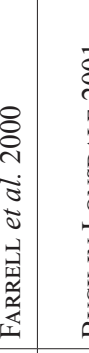 & 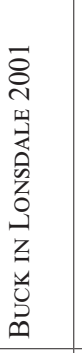 & 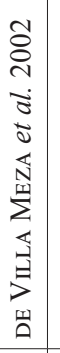 & 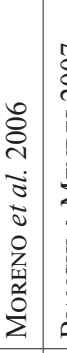 & 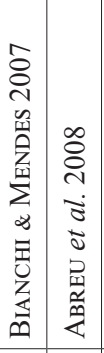 & 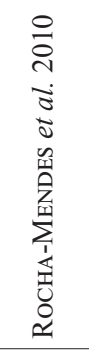 & 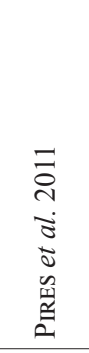 & 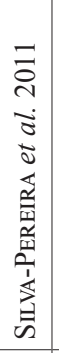 & 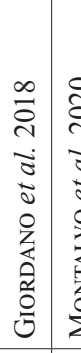 & 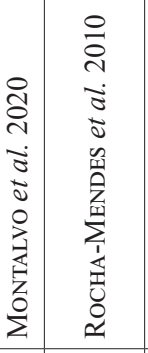 & 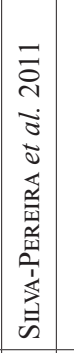 & 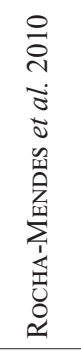 & 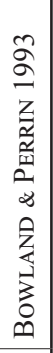 \\
\hline 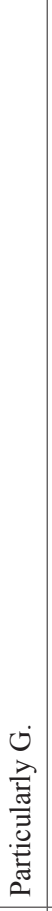 & 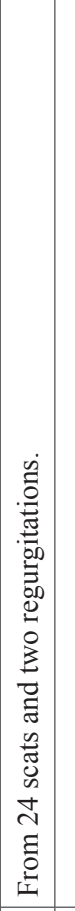 & 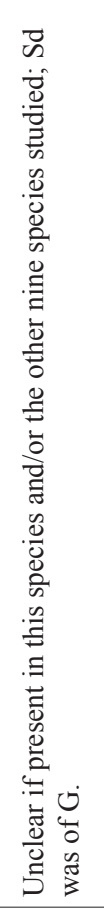 & 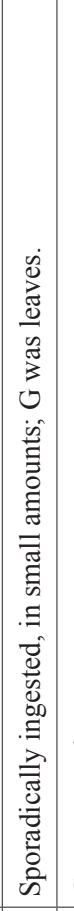 & 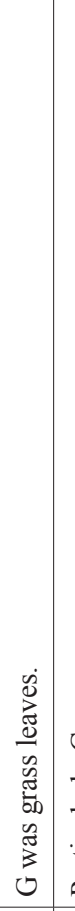 & 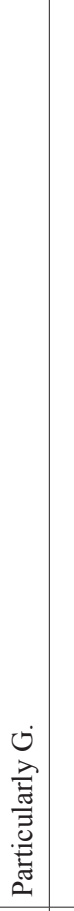 & & 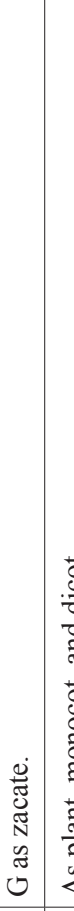 & 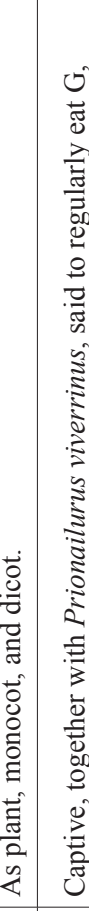 & 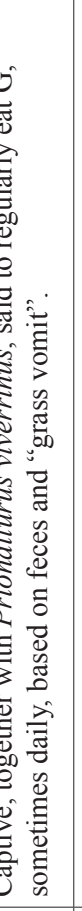 & & 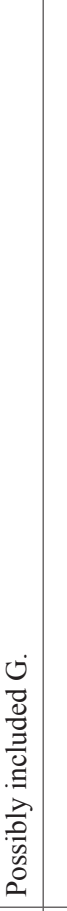 & & 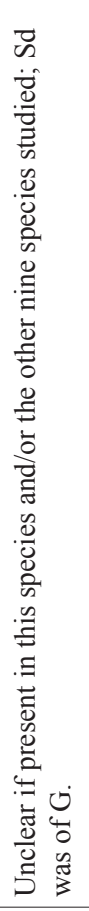 & 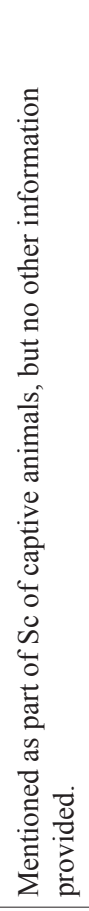 & 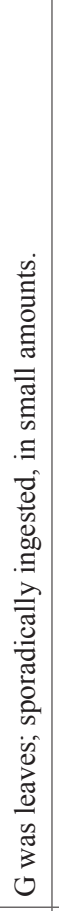 & $\begin{array}{c} \\
\\
\end{array}$ & 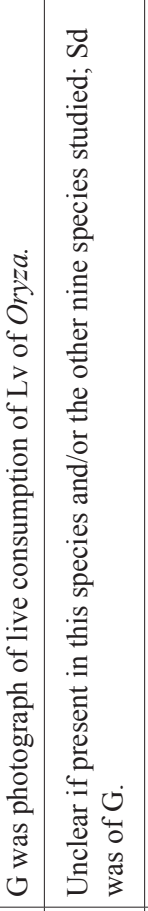 & 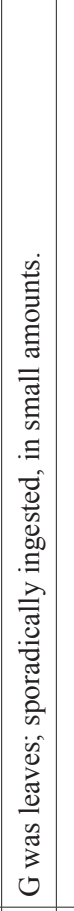 & 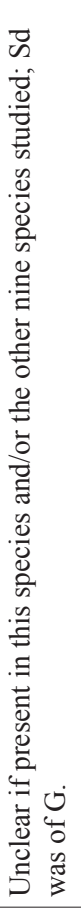 & 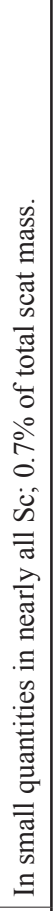 \\
\hline$\ddot{n}$ & $\begin{array}{l}0 \\
\tilde{n} \\
\tilde{n}\end{array}$ & $\ddot{n}$ & $\ddot{n}$ & $\ddot{\infty}$ & $\ddot{\curvearrowleft}$ & $\check{n}$ & $\ddot{n} \quad \delta$ & $\ddot{n}$ & ô & $\ddot{n}$ & $\ddot{\check{n}}$ & $\ddot{n} \ddot{n}$ & $\ddot{n}$ & $\ddot{n}$ & $\ddot{n}$ & $\ddot{n}$ & ô & $\ddot{n}$ & $\ddot{n}$ & $\ddot{n}$ \\
\hline ㅇ & i & \pm & $\ddot{m}$ & $q$ & $\circ:$ & $\sigma \delta$ & $\approx$ & 우 & 1 & $\vec{n}$ & તે & \begin{tabular}{l|l}
$\mathcal{8}$ \\
\end{tabular} & $a$ & 1 & $\because$ & \begin{tabular}{c|c}
$\infty$ & -
\end{tabular} & ฮิ & i & in & 8 \\
\hline o & O & 1 & 1 & 1 & O & O & 오 & o & 1 & 이 & 오 & 오 & 1 & 1 & I & 어 & 1 & । & 1 & 1 \\
\hline :̊े. & $\stackrel{\circ}{\stackrel{े}{ }}$ & \&ั. & ֶ̃ & $\ddot{凶}$ & ఏें & $\stackrel{\circ}{\Xi}$ & $\stackrel{\circ}{\circ}$ & ठ̊̊ & 1 & $\stackrel{\circ}{\infty}$ & iे & હें & ֻै & ֻै & 巡 & iे & ्ֶर & \% & ्ֶर & ्ֶर \\
\hline 3 & 0 & $\begin{array}{l}\vec{n} \\
\dot{n} \\
\dot{0}\end{array}$ & 0 & ט. & 30 & ט & o & a & 0 & ن & $\infty 2$ & $a$ & $\begin{array}{l}\vec{n} \\
\dot{n} \\
\dot{v}\end{array}$ & $a$ & 0 & c & $\begin{array}{l}\vec{n} \\
\text { in } \\
0\end{array}$ & ט & $\begin{array}{l}\vec{D} \\
\text { in } \\
v^{0}\end{array}$ & ט \\
\hline & & 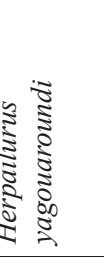 & & 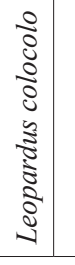 & & & & & & & & 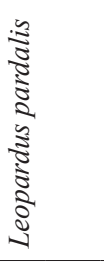 & & & & & 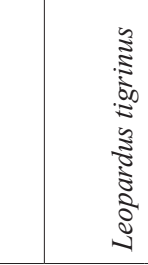 & & 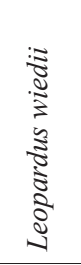 & 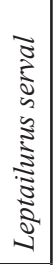 \\
\hline \multicolumn{21}{|c|}{ 它 } \\
\hline & & & & & & & & & & & & 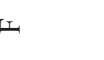 & & & & & & & & \\
\hline
\end{tabular}




\begin{tabular}{|c|c|c|c|c|c|c|c|c|c|c|c|c|c|c|c|c|c|c|c|c|c|c|}
\hline 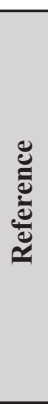 & 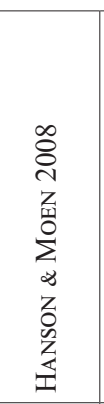 & 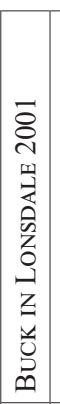 & 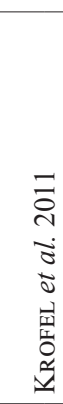 & & 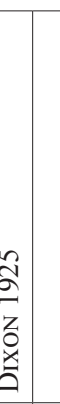 & 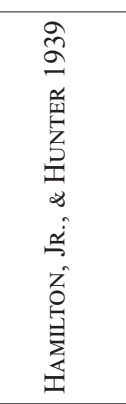 & 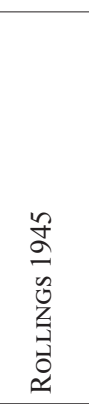 & 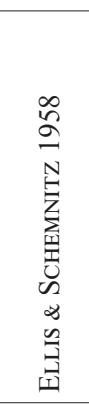 & 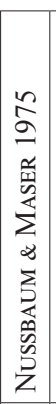 & 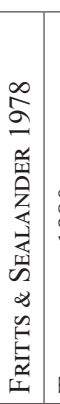 & 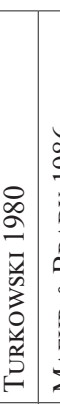 & \multicolumn{4}{|c|}{$\frac{\infty}{\sigma}$} & 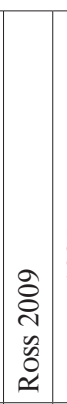 & 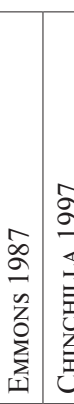 & 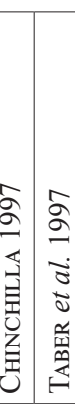 & 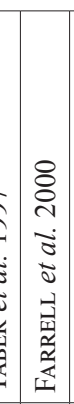 & 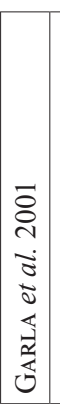 & 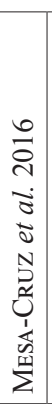 & 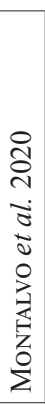 \\
\hline है & 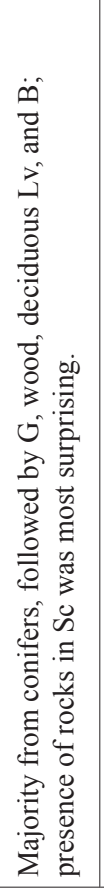 & 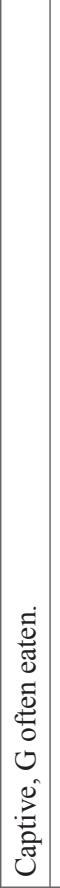 & 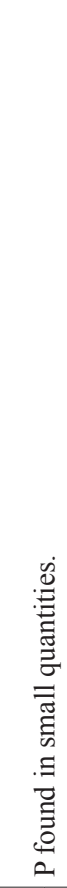 & & & 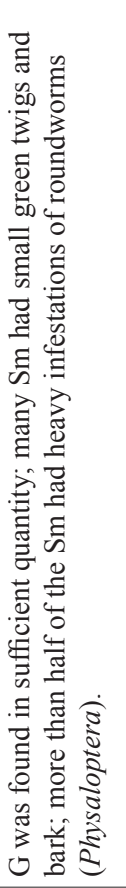 & 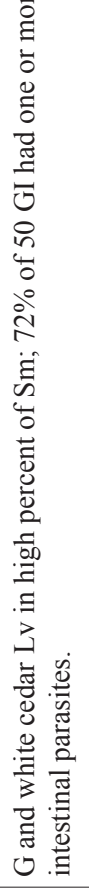 & 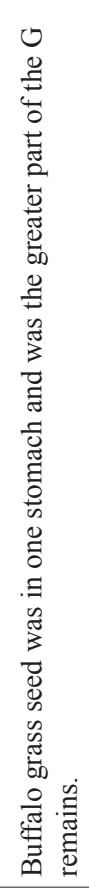 & & 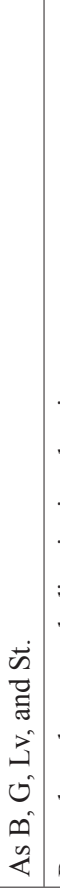 & 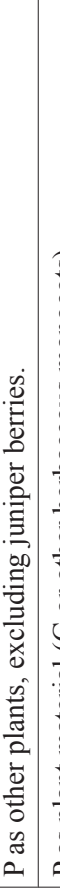 & 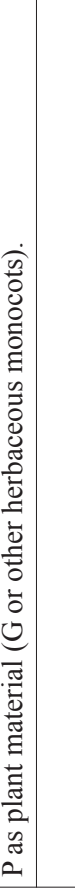 & & & & 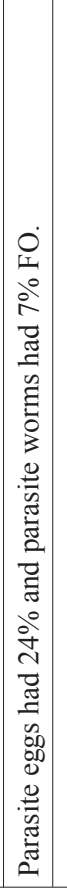 & & 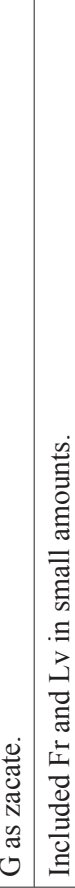 & 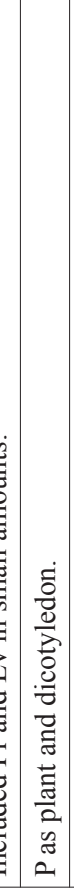 & 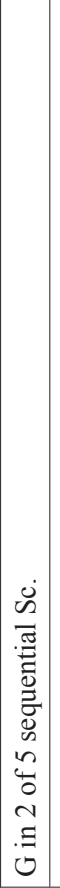 & & 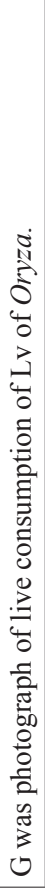 \\
\hline$\overline{5}$ & $\ddot{n}$ & ठ̊ & $\ddot{i s}$ & ह & ह & ह & ह & ઘ & $\ddot{\mathscr{L}}$ & ह & $\ddot{n}$ & घี & $\dot{n}$ & $\ddot{\check{n}}: \tilde{u}$ & $\begin{array}{l}0 \\
n\end{array}$ & $\ddot{\varkappa}$ & $\ddot{n}$ & $\ddot{\mathscr{L}}$ & $\dot{n}$ & $\ddot{\varkappa}$ & $\ddot{n}$ & है \\
\hline z & 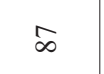 & 1 & g) & $\bar{m} \propto$ & $\stackrel{0}{\infty}$ & q & in & 으 & $\Xi$ & 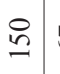 & 5 & $\stackrel{m}{\Rightarrow} \pm$ & \pm & + & $-r$ & 姜 & $\approx \approx$ & त) & $m$ & 의 & $a$ & - \\
\hline $\bar{\delta}$ & 이 & 1 & O & ol & $\frac{1}{\approx}$ & O & 1 & 이 & O & 1 & O) & O) & $\Sigma$ & $\Sigma \Sigma$ & $\Sigma \Sigma$ & $\begin{array}{l}0 \\
0\end{array}$ & $\begin{array}{lll}0 & 0 \\
\end{array}$ & \begin{tabular}{l|l}
0 & 0 \\
1
\end{tabular} & $\begin{array}{l}0 \\
0\end{array}$ & 足 & O & 1 \\
\hline$\check{8}$ & 高 & 1 & $\stackrel{\circ}{=}$ & $\begin{array}{c}\stackrel{0}{\circ} \\
\text { ì }\end{array}$ & $\stackrel{\text { ¿े }}{\circ}$ & 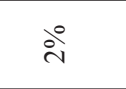 & $\ddot{凶}$ & ఏें & $\infty$ & 峞 & $\stackrel{\circ}{0}$ & 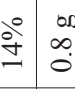 & $\begin{array}{ccc}0 \\
0 \\
\vdots \\
\vdots\end{array}$ & 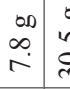 & 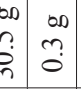 & $\underset{\stackrel{\circ}{\sim}}{\sim}$ & 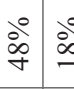 & 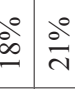 & $\begin{array}{l}0 \\
0 \\
0\end{array}$ & 兽 & さें & 1 \\
\hline Uే & $>$ & ن & a & $\left.\alpha\right|_{\bar{\nu}} ^{\bar{\nu}}$ & $\begin{array}{l}\vec{v} \\
\overrightarrow{8}\end{array}$ & ט & $\begin{array}{l}> \\
0\end{array}$ & 0 & $>$ & $>$ & a & o & $\overrightarrow{3}$ & 3 & $>>$ & $>$ & ن & بـ & $-\infty$ & $\infty$ & 0 & ט \\
\hline 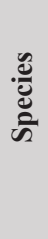 & 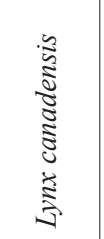 & & 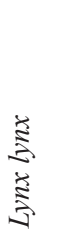 & & & & & & 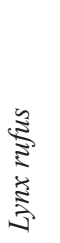 & & & & & & & 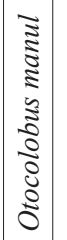 & & & 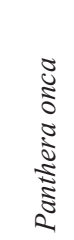 & & & \\
\hline$\sum_{\Sigma}$ & & & & & & & & & & 0 & & & & & & & & & & & & \\
\hline 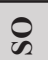 & & & & & & & & & & L & & & & & & & & & & & & \\
\hline
\end{tabular}




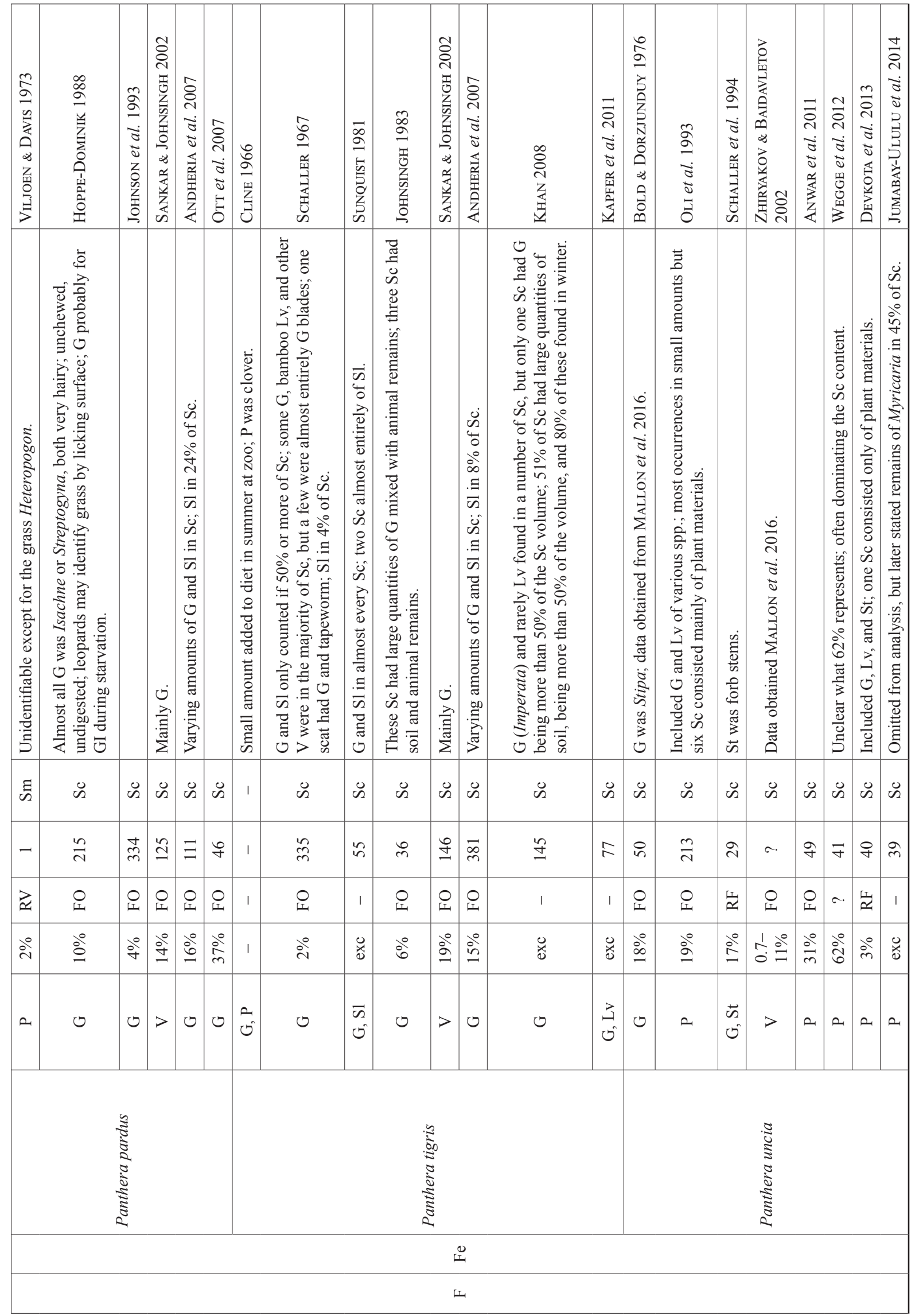




\begin{tabular}{|c|c|c|c|c|c|c|c|c|c|c|c|c|c|c|c|c|c|c|c|c|c|c|}
\hline 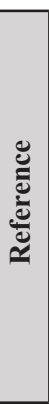 & 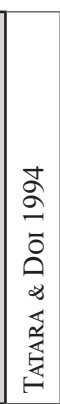 & 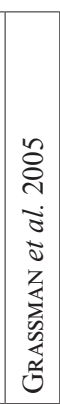 & 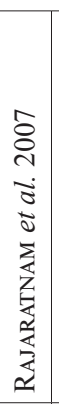 & 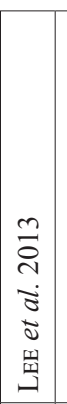 & 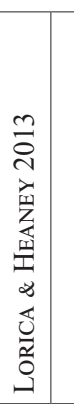 & 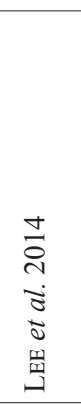 & 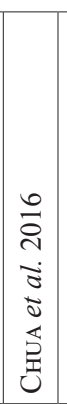 & 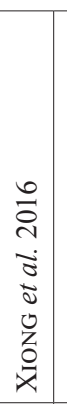 & 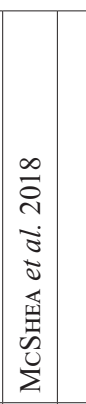 & 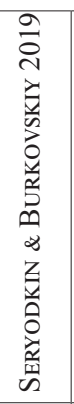 & 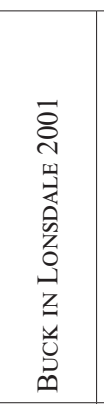 & 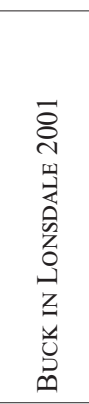 & 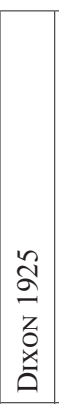 & 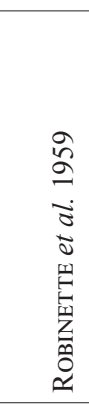 & & 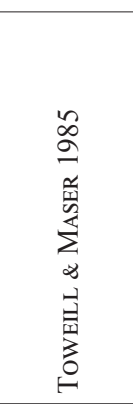 & 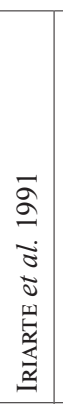 & 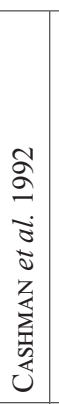 & 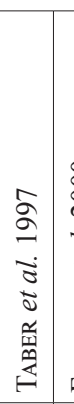 & 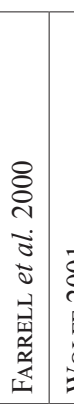 & 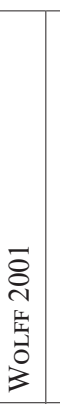 & 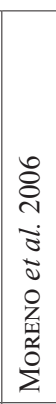 \\
\hline z⿺ & 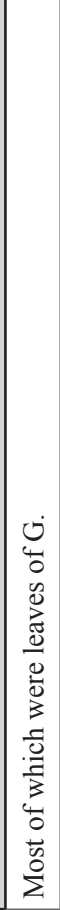 & & & 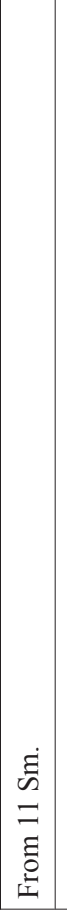 & & 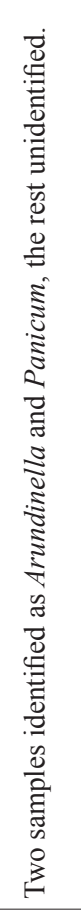 & 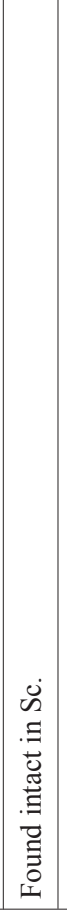 & 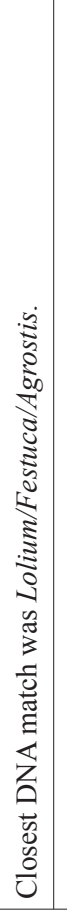 & 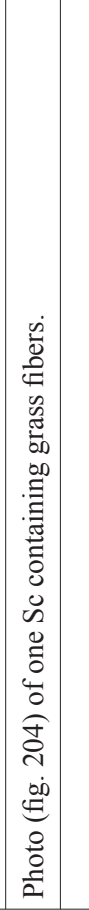 & 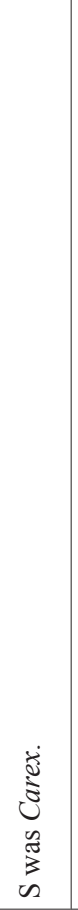 & 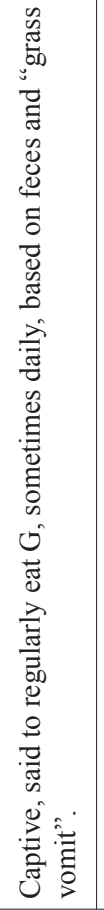 & 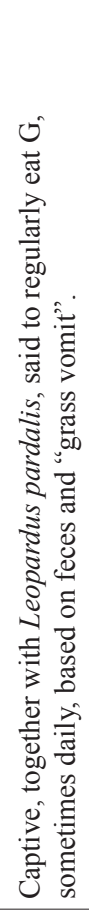 & & 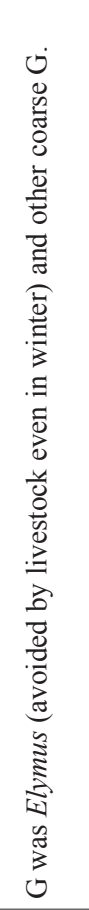 & 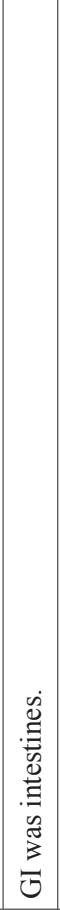 & 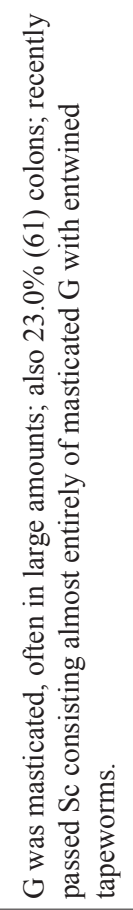 & & 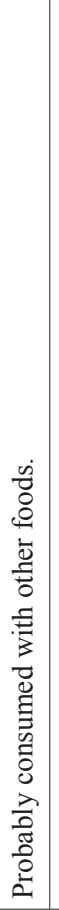 & 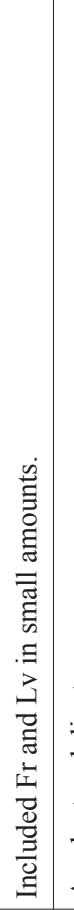 & 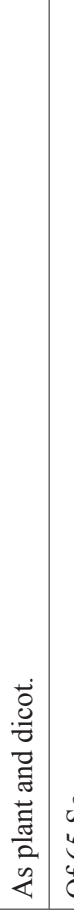 & 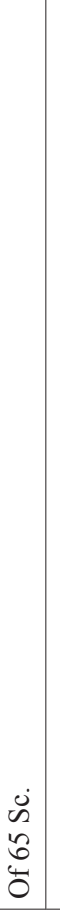 & 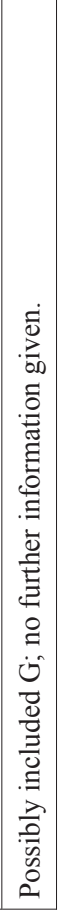 \\
\hline$\vec{F}$ & $\ddot{n}$ & $\ddot{n}$ & $\ddot{\mathscr{n}}$ & \pm & $\ddot{n}$ & $\ddot{n}$ & $\ddot{n}$ & $\ddot{n}$ & $\ddot{n}$ & $\ddot{n}$ & ठ̊ & ठ̊ & ह & ઘ & $\begin{array}{l}\dot{n} \\
\tilde{w}\end{array}$ & घ & $\ddot{n}$ & $\ddot{n}$ & $\ddot{\mathscr{n}}$ & $\check{\mathscr{n}}$ & \pm & $\ddot{n}$ \\
\hline z & in & $n$ & $\approx$ & in & $\vec{n}$ & $\underset{\sim}{\infty}$ & 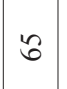 & F & - & $\vec{I}$ & 1 & 1 & $q$ & $\stackrel{\sim}{\sim}$ & $\hat{\mathbf{A}}$ & $\bar{\sigma}$ & $\ddot{q}$ & ڤે & $\approx$ & $+\delta$ & $\dot{\infty}$ & $\infty$ \\
\hline $\bar{\delta}$ & o & O & o & o & O & 足 & 足 & O & । & 오 & 1 & 1 & 이 & O & o & 이 & o & o & o & o & O & O \\
\hline$\check{8}$ & $\stackrel{2}{\circ}$ & ذి & $\stackrel{\circ}{ٍ}$ & aे & $\stackrel{\circ}{m}$ & ڤे & 离 & in & 1 & ळे & 1 & I & $\stackrel{\circ}{\circ}^{\circ}$ & ذें & 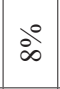 & $\stackrel{\circ}{\varrho}$ & iे & 吕 & $\stackrel{\stackrel{\partial}{ \pm}}{=}$ & $\stackrel{\circ}{\circ}$ & $\stackrel{\circ}{\stackrel{\circ}{~}}$ & in \\
\hline Uే & $a$ & ט & ט & $a$ & ט & $a$ & ט & 0 & 0 & $\begin{array}{l}n \\
0 \\
0\end{array}$ & ט & ט & ט & ט & 0 & 0 & $a$ & $\begin{array}{l}3 \\
0 \\
0\end{array}$ & $a$ & $a c$ & ט & is \\
\hline 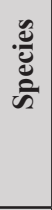 & & & & & & 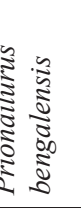 & & & & & 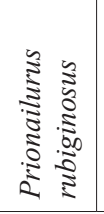 & 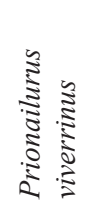 & & & & & & & & & & \\
\hline$\sum_{\Sigma}$ & & & & & & & & & & & & 东 & & & & & & & & & & \\
\hline o & & & & & & & & & & & & 工 & & & & & & & & & & \\
\hline
\end{tabular}




\begin{tabular}{|c|c|c|c|c|c|c|c|c|c|c|c|c|c|c|c|c|c|c|c|c|c|}
\hline 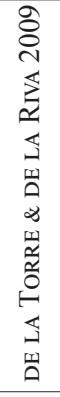 & 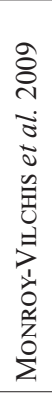 & 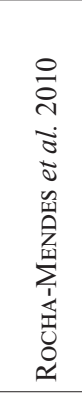 & 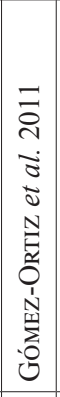 & 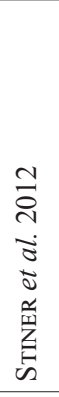 & 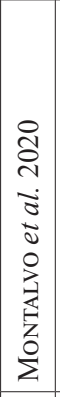 & 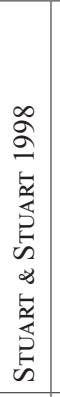 & 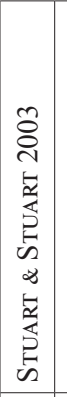 & 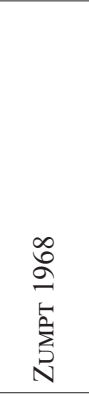 & 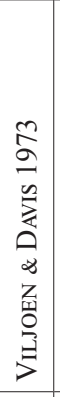 & 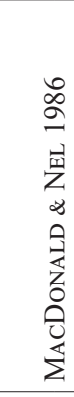 & & 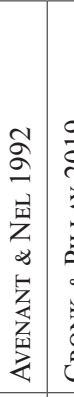 & 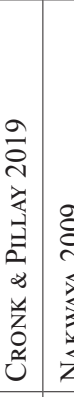 & 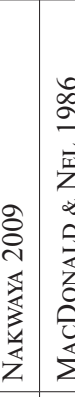 & 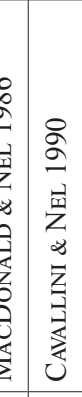 & 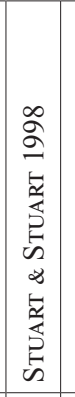 & 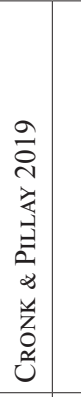 & 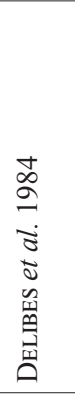 & 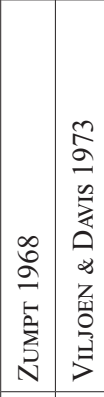 & 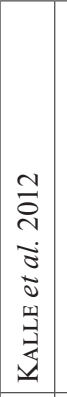 & 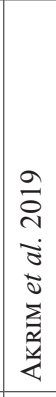 \\
\hline & & 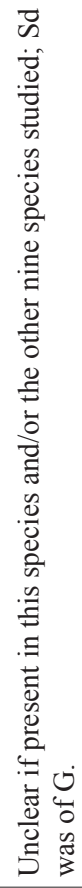 & & 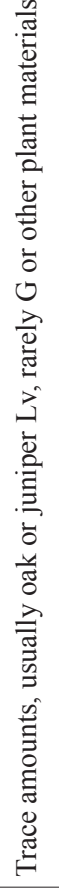 & 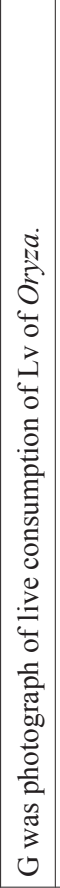 & & & 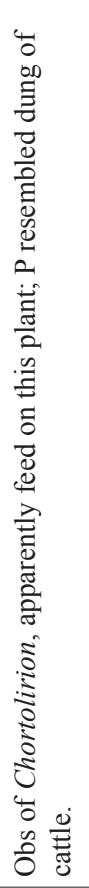 & & 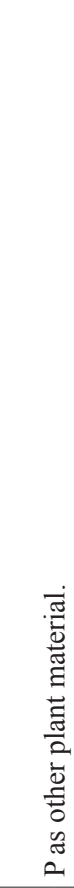 & & 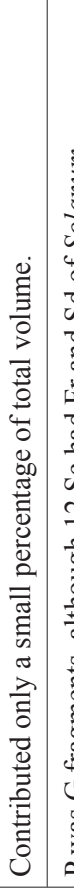 & 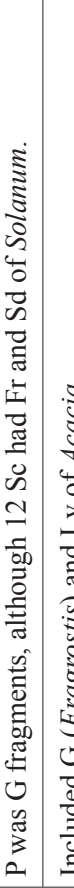 & \begin{tabular}{l|l} 
& \\
0 \\
0 \\
0
\end{tabular} & 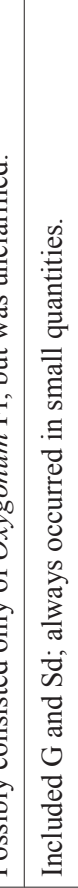 & & 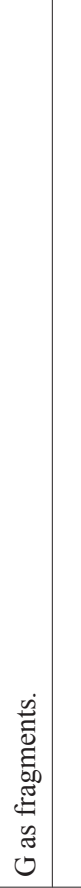 & 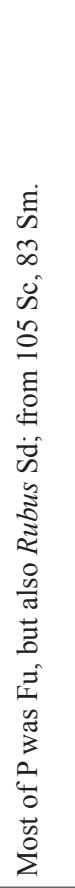 & 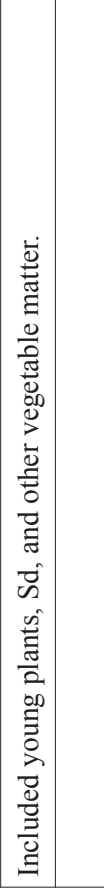 & 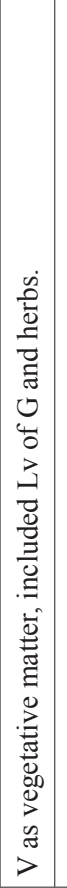 & 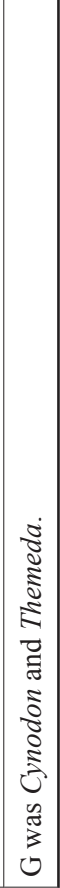 \\
\hline$\ddot{n}$ & $\ddot{n}$ & $\ddot{n}$ & $\ddot{n}$ & $\ddot{n}$ & ô & $\ddot{\mathscr{L}}$ & $\ddot{n}$ & हี & हี & $\ddot{\mathscr{L}}$ & & $\ddot{n} \delta$ & $\ddot{n}$ & $\ddot{n}$ & $\begin{array}{l}\dot{n} \\
2\end{array}$ & $\ddot{n}$ & $\ddot{n}$ & $\begin{array}{l}\text { E } \\
\text { un } \\
\tilde{n}\end{array}$ & 甜 & $\check{n}$ & $\ddot{n}$ \\
\hline$\stackrel{\infty}{m}$ & $\stackrel{\Xi}{\Xi}$ & 은 & $\stackrel{\infty}{\Omega}$ & $\approx$ & - & in & ㅇ & $\approx$ & - & in & & \begin{tabular}{l|l}
$\infty$ & $\wp$ \\
$\forall$ & $\wp$
\end{tabular} & 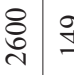 & $\begin{array}{lll}g & 0\end{array}$ & $\theta \underset{\sim}{+}$ & $\hat{\varrho}$ & 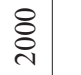 & $\stackrel{\infty}{\infty}$ & $\vec{N} \sim$ & $\begin{array}{l}\infty \\
\stackrel{2}{2}\end{array}$ & in \\
\hline 1 & o & 1 & O & Oે & 1 & o & O & o & $\vec{a}$ & 이 & & O & \begin{tabular}{l|l}
0 & 0 \\
\end{tabular} & 企任 & \begin{tabular}{l|l} 
& I \\
\end{tabular} & $\begin{array}{l}0 \\
0\end{array}$ & O) & ○ & 足 & $\mid \begin{array}{l}0 \\
1\end{array}$ & $\stackrel{x}{\simeq}$ \\
\hline ्ֶर & 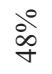 & $\ddot{\mathscr{x}}$ & $\stackrel{\circ}{\circ}$ & ठ̊े & 1 & ळे & $\begin{array}{l}0 \\
\infty \\
\sim\end{array}$ & $\stackrel{\circ}{=}$ & $\begin{array}{l}\mathscr{\Xi} \\
\mathbb{\Xi}\end{array}$ & $\stackrel{\circ}{\stackrel{\circ}{\sigma}}$ & $\stackrel{े}{\circ}$ & సें & هั & bें & $\stackrel{\circ}{\circ}$ & $\stackrel{2}{i}$ & 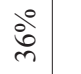 & ले & $\stackrel{\stackrel{\square}{ \pm}}{\stackrel{\circ}{\exists}}$ & वें & in \\
\hline$a$ & 0 & $\begin{array}{l}\vec{n} \\
\tilde{n} \\
\tilde{0}\end{array}$ & 0 & $>$ & ט & 0 & 0 & $a$ & $a$ & $\begin{array}{l}\vec{n} \\
0\end{array}$ & $a$ & $a$ & $a$ & $\begin{array}{l}\vec{u} \\
\vec{\Delta}\end{array}$ & 20 & ن & 0 & $a$ & \begin{tabular}{l|l}
$\vec{n}$ \\
si
\end{tabular} & $>$ & 0 \\
\hline \multicolumn{6}{|c|}{ 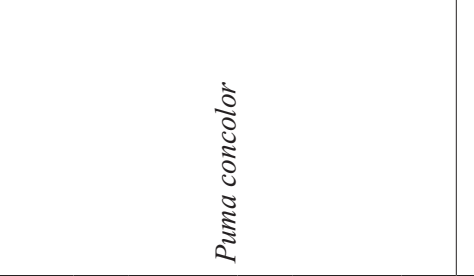 } & $\begin{array}{l}5 \\
5 \\
0 \\
0 \\
0\end{array}$ & 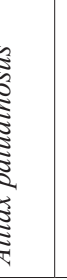 & \multicolumn{6}{|c|}{ 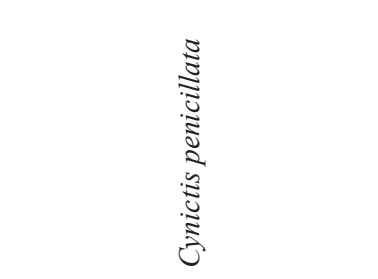 } & 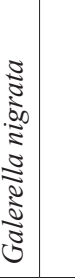 & \multicolumn{2}{|c|}{ 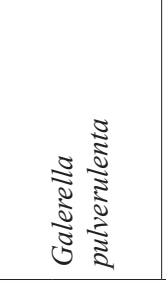 } & 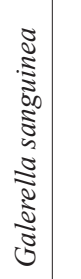 & 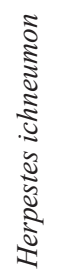 & 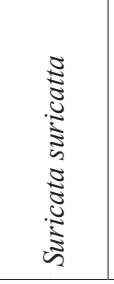 & \multicolumn{2}{|c|}{ 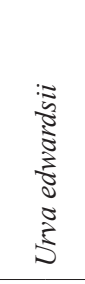 } \\
\hline \multicolumn{6}{|c|}{ 证 } & \multicolumn{16}{|c|}{$\stackrel{\Xi}{I}$} \\
\hline & & & & & & & & & & 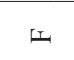 & & & & & & & & & & & \\
\hline
\end{tabular}




\begin{tabular}{|c|c|c|c|c|c|c|c|c|c|c|c|c|c|c|c|c|c|c|}
\hline 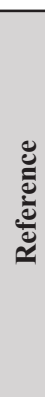 & $\begin{array}{l}n \\
0 \\
a \\
z \\
z \\
0 \\
0 \\
0\end{array}$ & 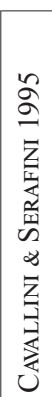 & 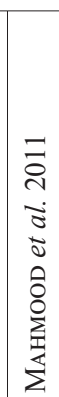 & 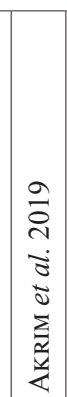 & 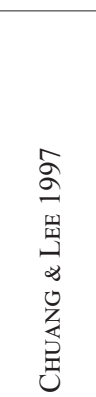 & 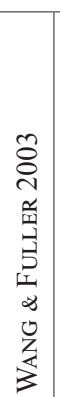 & 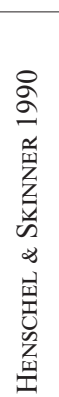 & 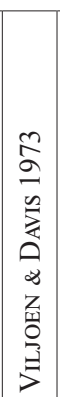 & 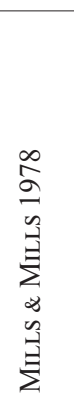 & 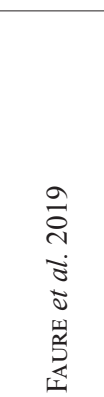 & 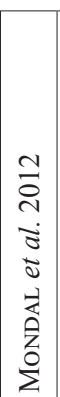 & 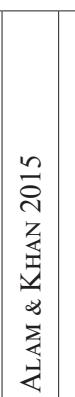 & 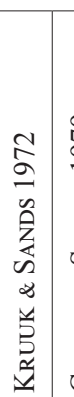 & 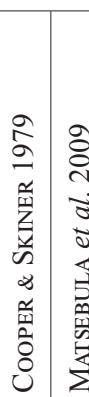 & $\begin{array}{l}\hat{\mathbf{2}} \\
\mathbf{3} \\
\mathbf{5}\end{array}$ & 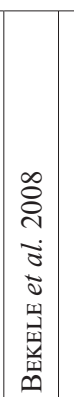 & 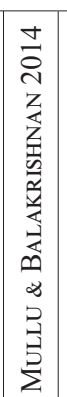 & 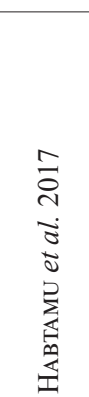 \\
\hline$\frac{8}{0}$ & 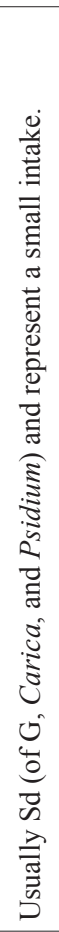 & & 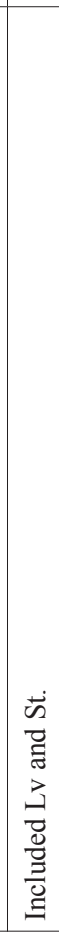 & 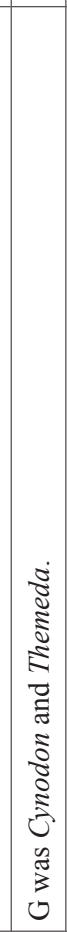 & 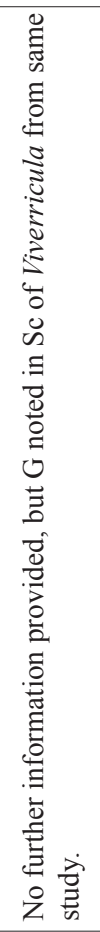 & & 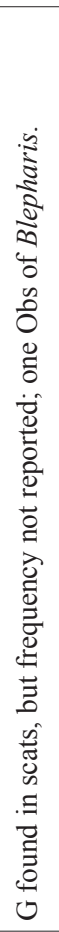 & 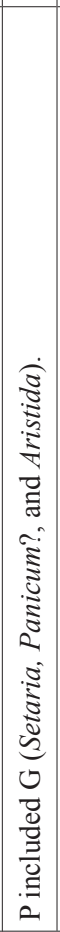 & 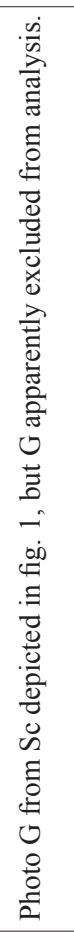 & 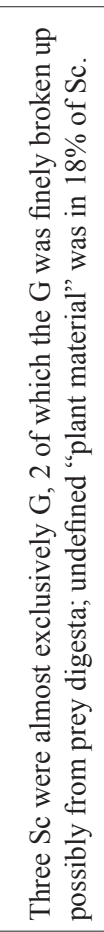 & 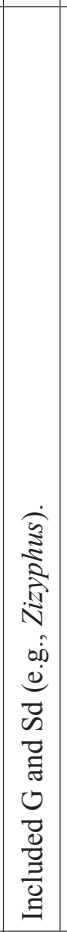 & 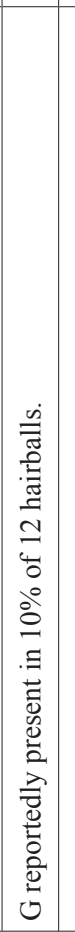 & 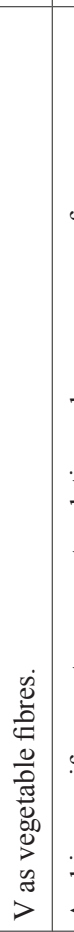 & 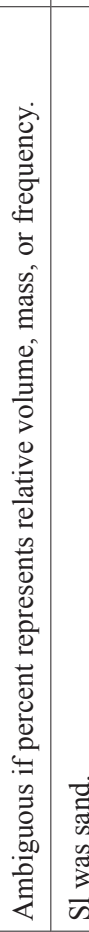 & 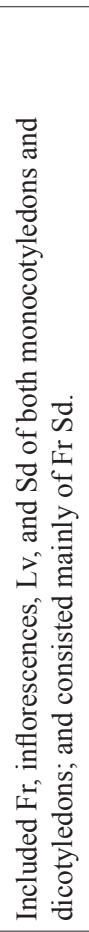 & 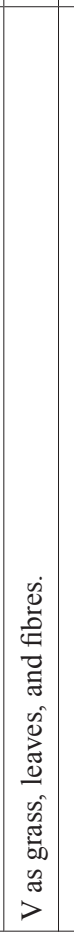 & 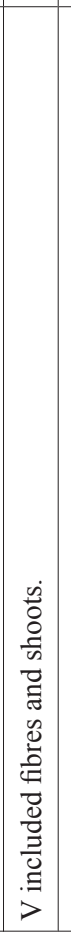 & 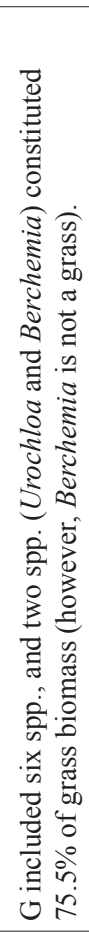 \\
\hline है & $\ddot{n}$ & $\ddot{n}$ & $\ddot{n}$ & $\ddot{n}$ & $\ddot{n}$ & $\ddot{n}$ & $\ddot{n}$ & घี & $\ddot{n}$ & $\ddot{n}$ & $\ddot{n}$ & $\ddot{n}$ & $\ddot{n}$ & $\ddot{n}$ & $\ddot{n}$ & \pm & $\ddot{n}$ & $\ddot{n}$ \\
\hline z & 守 & $\stackrel{7}{\beth}$ & i & 8 & ๙ิ & $\cong$ & $\approx$ & - & $\stackrel{\infty}{\infty}$ & $\stackrel{\infty}{\Xi}$ & $\stackrel{\infty}{\infty}$ & $\tilde{\infty}$ & $\stackrel{2}{2}$ & $\vec{\infty} \propto$ & à & $\bar{n}$ & $\stackrel{\infty}{i n}$ & $\underset{\sim}{\widetilde{N}}$ \\
\hline$\overline{8}$ & O & o & O & $\frac{\pi}{\alpha}$ & O & O & I & $\vec{a}$ & I & O & $\begin{array}{l}0 \\
0\end{array}$ & \begin{tabular}{l|l}
0 \\
\end{tabular} & O & \begin{tabular}{l|l} 
a. & 0 \\
II
\end{tabular} & 이 & $\begin{array}{l}0 \\
\end{array}$ & 이 & $\sum_{\Omega}$ \\
\hline ठ & ふें & ลे & 离 & ठे & $\infty_{\infty}^{\circ}$ & $\stackrel{\circ}{\circledR}$ & ्ֶ. & iें & ्ֶ. & ৯े & $\stackrel{\circ}{\stackrel{0}{2}}$ & $\stackrel{\circ}{\stackrel{2}{1}}$ & 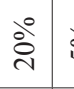 & in & $\frac{\circ}{\infty}$ & $亠_{\infty}^{\circ}$ & $\stackrel{\circ}{\circ}$ & ळे \\
\hline లँ & $a$ & $>$ & a & 0 & $a$ & 0 & 0 & $a$ & 0 & ט & $>$ & 0 & $>$ & $\begin{array}{l}\bar{n} \\
0 \\
0\end{array}$ & $>$ & $>$ & $\begin{array}{l}> \\
3\end{array}$ & ט \\
\hline 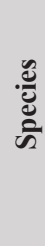 & & 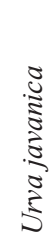 & & & 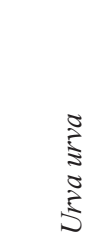 & & 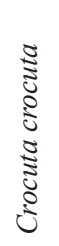 & & & & 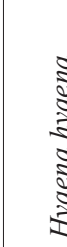 & & & 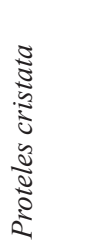 & & 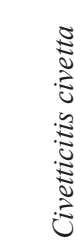 & है & \\
\hline$\sum$ & \multicolumn{6}{|c|}{$\stackrel{\Xi}{ \pm}$} & \multicolumn{8}{|c|}{ 坣 } & \multicolumn{4}{|c|}{5} \\
\hline \& & \\
\hline
\end{tabular}




\begin{tabular}{|c|c|c|c|c|c|c|c|c|c|c|c|c|c|c|c|c|c|c|c|c|}
\hline 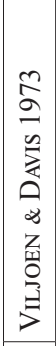 & 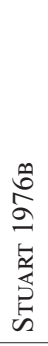 & 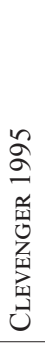 & 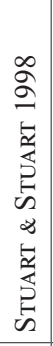 & & 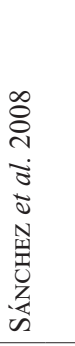 & 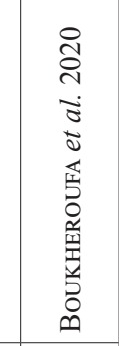 & 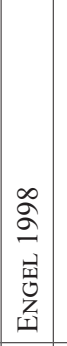 & 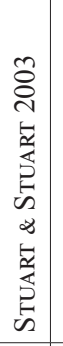 & 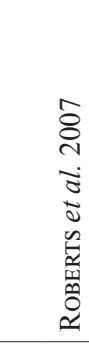 & & 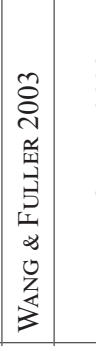 & 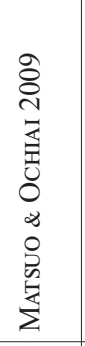 & 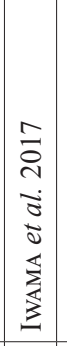 & 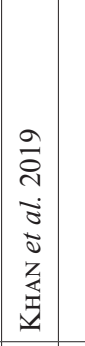 & 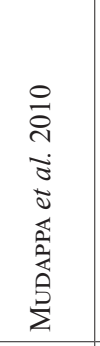 & 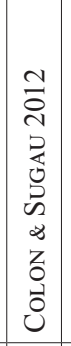 & 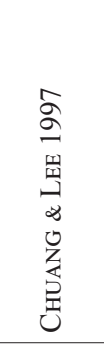 & 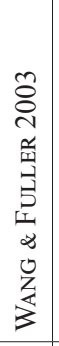 & 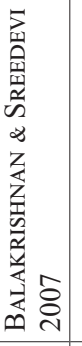 & 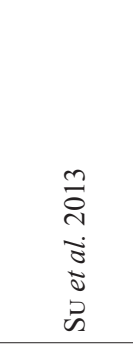 \\
\hline & 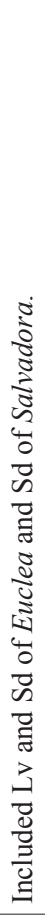 & & 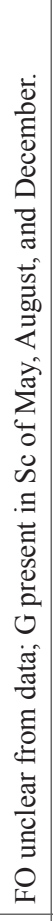 & & 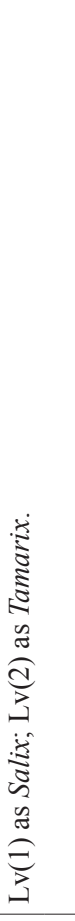 & 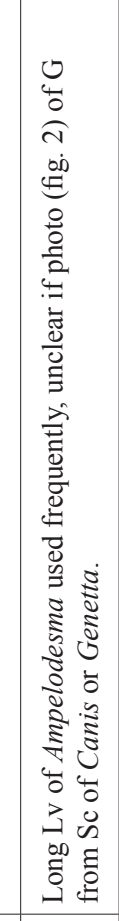 & 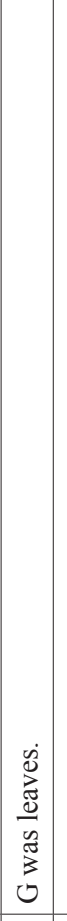 & & 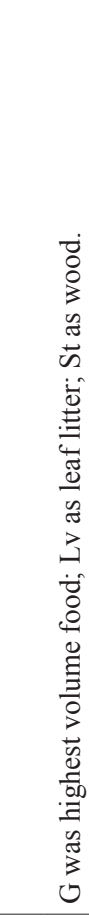 & & & 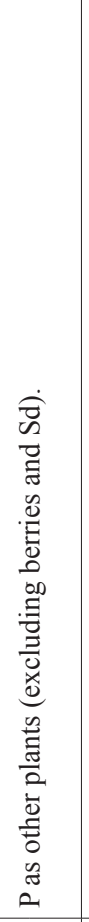 & 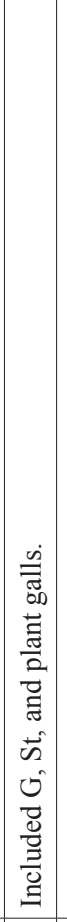 & & 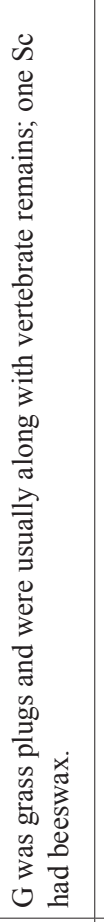 & & 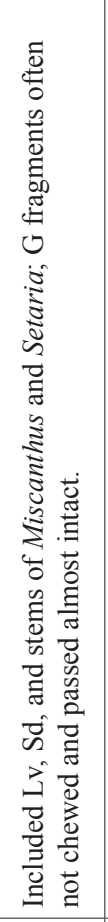 & & 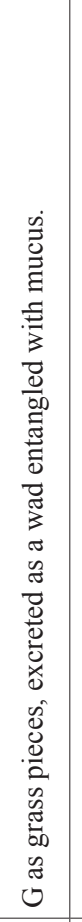 & 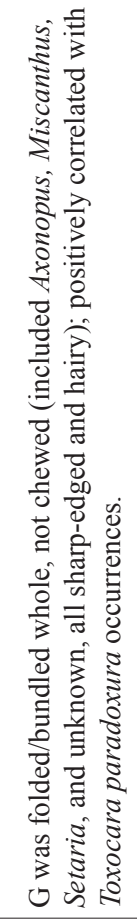 \\
\hline हี & $\ddot{n}$ & $\ddot{n}$ & $\ddot{n}$ & & $\ddot{n}$ & $\ddot{n}$ & $\ddot{\leftrightarrow}$ & $\ddot{n}$ & $\ddot{n}$ & & $\ddot{n}$ & $\Xi$ & $\ddot{E}$ & $\ddot{n}$ & $\ddot{n}$ & $\ddot{n}$ & $\ddot{n}$ & $\ddot{n}$ & हี & $\ddot{n}$ \\
\hline- & $\cong$ & $\stackrel{m}{n}$ & $\tilde{n}$ & & 8 & i & $\frac{n}{n}$ & $\stackrel{\infty}{-}$ & $\stackrel{N}{m}$ & & $\hat{n}$ & $\hat{\imath}$ & $\approx$ & $\cong$ & $\stackrel{m}{\stackrel{m}{\Xi}}$ & $\infty$ & 壳 & F & $r$ & s్ \\
\hline$\gtrsim$ & o & O) & o & & 이 & 1 & $\begin{array}{l}0 \\
0 \\
1\end{array}$ & o & 이 & & 이 & 이 & $\begin{array}{l}0 \\
1\end{array}$ & $\frac{\pi}{2}$ & O & O & O & 이 & 이 & O \\
\hline$\stackrel{\circ}{\circ}$ & ฉ̊ & $\stackrel{\infty}{\infty}$ & 1 & 吕 & ڤ̊ำ & 1 & 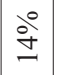 & ذें & ذి పें & $\begin{array}{c}0 \\
:\end{array}$ & $\stackrel{\circ}{\circ}$ & $\therefore$ & ळे & $\stackrel{\circ}{\dot{\sigma}}$ & $\stackrel{\circ}{-}$ & $\frac{\partial^{\circ}}{\vec{\lambda}}$ & 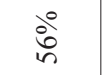 & $\stackrel{\partial^{0}}{\vec{\sim}}$ & $\stackrel{\circ}{\stackrel{े}{\sigma}}$ & ஓे \\
\hline a & $a$ & 0 & 0 & 0 & 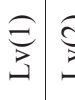 & 0 & ن & 0 & (ב) & $\vec{n}$ & ט & 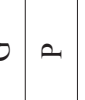 & $a$ & 0 & 0 & 3 & $a$ & 3 & 0 & 0 \\
\hline & & & & 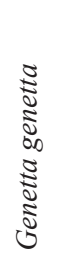 & & & 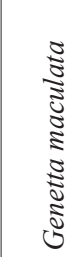 & & 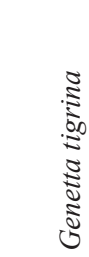 & & & 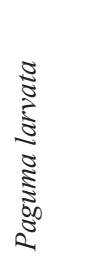 & & 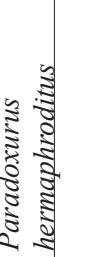 & 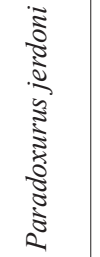 & 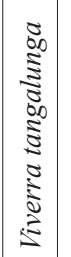 & & & 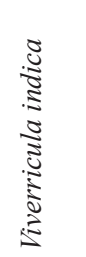 & \\
\hline \multicolumn{21}{|c|}{5} \\
\hline & & & & & & & & & & & & & & & & & & & & \\
\hline
\end{tabular}




\section{References}

Abreu K.C., Moro-Rios R.F., Silva-Pereira J.E., Miranda J.M., Jablonski E.F. \& Passos F.C. (2008). Feeding habits of ocelot (Leopardus pardalis) in Southern Brazil. Mammalian Biology 73: 407-411. https://doi.org/10.1016/j.mambio.2007.07.004

Ackerson B.K. \& Harveson L.A. (2006). Characteristics of a ringtail (Bassariscus astutus) population in Trans Pecos, Texas. Texas Journal of Science 8: 169-184.

Agnarsson I., Kuntner M. \& May-Collado L.J. (2010). Dogs, cats, and kin: a molecular specieslevel phylogeny of Carnivora. Molecular Phylogenetics and Evolution 54: 726-745.

https://doi.org/10.1016/j.ympev.2009.10.033

Aguiar L.M., Moro-Rios R.F., Silvestre T., Silva-Pereira J.E., Bilski D.R., Passos F.C., SeKiama M.L. \& Rocha V.J. (2011). Diet of brown-nosed coatis and crab-eating raccoons from a mosaic landscape with exotic plantations in southern Brazil. Studies on Neotropical Fauna and Environment 46: 153-161. https://doi.org/10.1080/01650521.2011.640567

Akrim F., Mahmood T., Nadeem M.S., Qasim S., Andleeb S. \& Fatima H. (2019). Distribution, dietary breadth and niche overlap between two sympatric mongoose species inhabiting Pir Lasura National Park, Azad Jammu and Kashmir, Pakistan. Pakistan Journal of Zoology 51: 1497-1507.

ALAM M.S. \& KHAN J.A. (2015). Food habits of striped hyena (Hyaena hyaena) in a semi-arid conservation area of India. Journal of Arid Land 7: 860-866. https://doi.org/10.1007/s40333-015-0007-2

Alexander L.F., Verts B.J. \& Farrell T.P. (1994). Diet of ringtails (Bassariscus astutus) in Oregon. Northwestern Naturalist 75: 97-101. https://doi.org/10.2307/3536831

Allen L., Goullet M. \& Palmer R. (2012). The diet of the dingo (Canis lupus dingo and hybrids) in north-eastern Australia: a supplement to the paper of Brook and Kutt (2011). The Rangeland Journal 34: 211-217. https://doi.org/10.1071/RJ11092

Álvarez-Castañeda S.T. \& GonzÁlez-Quintero P. (2005). Winter-spring food habits of an island population of coyote Canis latrans in Baja California, México. Journal of Arid Environments 60: 397404. https://doi.org/10.1016/j.jaridenv.2004.07.007

Alves-Costa C.P., da Fonseca G.A.B. \& Christófaro C. (2004). Variation in the diet of the brownnosed coati (Nasua nasua) in southeastern Brazil. Journal of Mammalogy 85: 478-482.

Amaral C. (2007). Dieta de duas espécies carnívoras simpátricas (graxaim-do-mato Cerdocyon thous (Linnaeus, 1766) e quati Nasua nasua (Linnaeus, 1766), nos municípios de TIjucas do sul e agudos do sul, Estado do Paraná. Ph.D. dissertation. Universidade Federal do Paraná, Paraná, Brazil.

Andelt W.F., Kie J.G., Knowlton F.F. \& Cardwell K. (1987). Variation in coyote diets associated with season and successional changes in vegetation. Journal of Wildlife Management 51: 273-277. https://doi.org/10.2307/3801002

Anderson P.C. \& Sigaut F. (2014). Introduction: Reasons for Variability in Harvesting Techniques and Tools. In: van Gijn A., Whittaker J. \& Anderson P.C. (eds) Exploring and Explaining Diversity in Agricultural Technology: 85-92. Oxbow Books, Oxford and Philadelphia.

ANDERSONe Ž. (1998). Summer nutrition of wolf (Canis lupus) in the Slitere Nature Reserve, Latvia. Proceedings of the Latvian Academy of Sciences, section B 52: 79-80.

Andersone Ž. \& Ozolinš J. (2004). Food habits of wolves Canis lupus in Latvia. Acta Theriologica 49: 357-367. https://doi.org/10.1007/BF03192534 
Andheria A.P., Karanth K.U. \& Kumar N.S. (2007). Diet and prey profiles of three sympatric large carnivores in Bandipur Tiger Reserve, India. Journal of Zoology 273: 169-175.

https://doi.org/10.1111/j.1469-7998.2007.00310.x

Anwar M.B., Jackson R., Nadeem M.S., JanečKa J.E., Hussain S., Beg M.A., Muhammad G. \& QAYYUM M. (2011). Food habits of the snow leopard Panthera uncia (Schreber, 1775) in Baltistan, northern Pakistan. European Journal of Wildife Research 57: 1077-1083.

https://doi.org/10.1007/s10344-011-0521-2

Anwar M.B., Nadeem M.S., Shah S.I., Kiayani A.R. \& Mushtaq M. (2012). A note on the diet of Indian wolf (Canis lupus) in Baltistan, Pakistan. Pakistan Journal of Zoology 44: 588-591.

AрÁтHY M.T. (1998). Data to the diet of the urban stone marten (Martes foina Erxleben) in Budapest. Opuscula Zoologica Budapest 31: 113-118.

Aragona M. \& Setz E.Z.F. (2001). Diet of the maned wolf, Chrysocyon brachyurus (Mammalia: Canidae), during wet and dry seasons at Ibitipoca State Park, Brazil. Journal of Zoology 254: 131-136. https://doi.org/10.1017/S0952836901000620

Arnaud G. \& Acevedo M. (1990). Hábitos alimenticios de la zorra gris Urocyon cinereoargenteus (Carnivora: Canidae) en la región meridional de Baja California, México. Revista de Biologia Tropical 38: 497-500.

Arnaud G., Rodríguez A. \& Cárdenas S.A. (1994). El gato domestico (Felis catus), implicaciones de su presencia y alternativas para su erradicacion. In: Rugio A.O. \& Vera A.C. (eds) Las Isla Socorro, Reserva de la Biosfera Archipielago de Revillagigedo, Mexico: 319-328. Centro de Investigaciones Biologicas del Noroeste S.C., Sonora, Mexico.

Atkinson R.P.D., Macdonald D.W. \& Kamizola R. (2002). Dietary opportunism in side-striped jackals Canis adustus Sundevall. Journal of Zoology 257: 129-139.

https://doi.org/10.1017/S0952836902000729

Aurioles-Gamboa D. \& CAMACho-Ríos F.J. (2007). Diet and feeding overlap of two otariids, Zalophus californianus and Arctocephalus townsendi: Implications to survive environmental uncertainty. Aquatic Mammals 33: 315-326.

Avenant N.L. \& Nel J.A.J. (1992). Comparison of the diet of the yellow mongoose in a coastal and a Karoo area. South African Journal of Wildlife Research 22: 89-93.

AvenANT N.L. \& Nel J.A.J. (2002). Among habitat variation in prey availability and use by caracal Felis caracal. Mammalian Biology 67: 18-33. https://doi.org/10.1078/1616-5047-00002

BACON E.S. \& BurghaRdT G.M. (1976). Ingestive behaviors of the American black bear. Bears: Their Biology and Management 3: 13-25.

Bakaloudis D.E., Bontzorlos V.A., Vlachos C.G., Papakosta M.A., Chatzinikos E.G., Braziotis S.G., \& Kontsiotis V.J. (2015). Factors affecting the diet of the red fox (Vulpes vulpes) in a heterogeneous Mediterranean landscape. Turkish Journal of Zoology 39: 1151-1159.

Balakrishnan M. \& Sreedevi M.B. (2008). Husbandry and mangement of the small Indian civet Viverricula indica (É. Geoffroy Saint-Hilaire, 1803) in Kerala, India. Small Carnivore Conservation 36: $9-13$.

Baranovskaia T.N. \& Kolosov A.M. (1935). Pitanie Lisitsy (Vulpes vulpes L.) Zoologicheskii Zhurnal 14: 523-550. 
Barboza P.S., Allen M.E., Rodden M. \& Pojeta K. (1994). Feed intake and digestion in the maned wolf (Chrysocyon brachyurus): consequences for dietary management. Zoo Biology 13: 375-381. https://doi.org/10.1002/zoo.1430130410

BARELLI C. \& HufFMAn M.A. (2016). Leaf swallowing and parasite expulsion in Khao Yai white-handed gibbons (Hylobates lar), the first report in an Asian ape species. American Journal of Primatology 79: e22610. https://doi.org/10.1002/ajp.22610

Barrs V.R., Beatty J.A., Tisdall P.L.C., Hunt G.B., Gunew M., Nicoll R.G. \& Malik R. (1999). Intestinal obstruction by trichobezoars in five cats. Journal of Feline Medicine and Surgery 1: 199-207. https://doi.org/10.1053/jfms.1999.0042

Beaver B.V. (1981). Grass eating by carnivores. Veterinary Medicine, Small Animal Clinician 76: 968969.

Bekele T., Afework B. \& Balakrishnan M. (2008). Feeding ecology of the African civet Civettictis civetta in the Menagesha-Suba State Forest, Ethiopia. Small Carnivore Conservation 39: 19-24.

BelyK V.I. (1962). Materials in the winter diet of the Yakut ermine. Proceedings of the All-Union Scientific Research Institute of Animal Origin, and Furs 19: 221-229.

BERRY M.P.S. (1981). Stomach contents of bat-eared foxes, Otocyon megalotis, from the northern Transvaal. South African Journal of Wildlife Research 11: 28-30.

Best T.L., Hoditscheк B. \& Thomas H.H. (1981). Foods of coyotes (Canis latrans) in Oklahoma. The Southeastern Naturalist 26: 67-69. https://doi.org/10.2307/3671333

Beyer W.N., Connor E.E. \& Gerould S. (1994). Estimates of soil ingestion by wildlife. Journal of Wildlife Management 58: 375-382. https://doi.org/10.2307/3809405

BiAnCHI R. DE C. \& MENDES S.L. (2007). Ocelot (Leopardus pardalis) predation on primates in Caratinga Biological Station, southeast Brazil. American Journal of Primatology 69: 1173-1178.

https://doi.org/10.1002/ajp.20415

Biró Z., Lanszki J., Szemethy L., Heltai M. \& Randi E. (2005). Feeding habits of feral domestic cats (Felis catus), wild cats (Felis silvestris) and their hybrids: trophic niche overlap among cat groups in Hungary. Journal of Zoology 266: 187-196. https://doi.org/10.1017/S0952836905006771

Bisbal E.F.J. (1986). Food habits of some neotropical carnivores in Venezuela (Mammalia, Carnivora). Mammalia 50: 329-339.

Buone S.J., Brown W.Y. \& Price I.R. (2007). Grass eating patterns in the domestic dog, Canis familiaris. Recent Advances in Animal Nutrition in Australia 16: 45-49.

Bjone S.J., Brown W.Y. \& Price I. R. (2009). Maternal influence on grass-eating behavior in puppies. Journal of Veterinary Behavior 4: 97-98.

BlaCKHALl S. (1980). Diet of the eastern native-cat, Dasyurus viverrinus (Shaw), in southern Tasmania. Wildlife Research 7: 191-197. https://doi.org/10.1071/WR9800191

Bold A. \& DorJzunduy S. (1976). Report on snow leopards in the southern spurs of the Gobi Altai. Proceedings of the Institute of General and Experimental Biology - Ulaanbaatar 11: 27-43.

Bond R.M. (1939). Coyote food habits on the Lava Beds National Monument. The Journal of Wildlife Management 3: 180-198. https://doi.org/10.2307/3796101

Bоотн D. (1835). An Analytical Dictionary of the English Language. James Cochrane and Co., London. 
Bošković I., Š́peranda M., Florijančić T., Šprem N., Ozimec S., DegmečIC D. \& Jelkić D. (2013). Dietary habits of the golden jackal (Canis aureus L.) in the eastern Croatia. Agriculturae Conspectus Scientificus 78: 245-248.

Botнma J. DU P. (1966). Food of the silver fox Vulpes chama. African Zoology 2: 205-209. https://doi.org/10.1080/00445096.1966.11447343

Bothma J. DU P. (1971). Food of Canis mesomelas in South Africa. Zoologica Africana 6: 195-203.

Boukheroufa M., Sakraoui F., Belbel, F. \& Sakraoui R. (2020). Winter diet of the common genet, Genetta genetta (Carnivora, Viverridae), and the African golden wolf, Canis anthus (Carnivora, Canidae), in altitudinal locality of the Edough Forest (northeastern Algeria). Zoodiversity 54: 67-74.

Bouroş G. \& Murariu D. (2017). Comparative diet analysis of the Eurasian otter (Lutra lutra) in different habitats: Putna-Vrancea Natural Park and Lower Siret Valley, south-eastern Romania. NorthWestern Journal of Zoology 13: 311-319.

Bowland J.M. \& Perrin M.R. (1993). Diet of serval Felis serval in a highland region of Natal. South African Journal of Zoology 28: 132-135.

Brahmi K., Mostefaoui O., Doumandi S., Baziz B. \& Aulagnier S. (2012). First quantitative data on the diet of the fennec fox, Vulpes zerda (Canidae, Carnivora), in Algeria. Folia Zoologica 61: 61-71. https://doi.org/10.25225/fozo.v61.i1.a10.2012

Brito J.C., Acosta A.L., Álvares F. \& Cuzin F. (2009). Biogeography and conservation of taxa from remote regions: An application of ecological-niche based models and GIS to North-African Canids. Biological Conservation 142: 3020-3029. https://doi.org/10.1016/j.biocon.2009.08.001

Bueno A. De A. \& MotTA-Junior J.C. (2004). Food habits of two synoptic canids, the maned wolf (Chrysocyon brachyurus) and the crab-eating fox (Cerdoycon thous), in southeastern Brazil. Revista Chilena de Historia Natural 77: 5-14.

Bukhtiyarov Y.A., Frost K.J. \& Lowry L.F. (1984). New information on foods of the spotted seal, Phoca largha, in the Bering Sea in spring. In: FAY F.H. \& Fedoseev G.A. (eds) Soviet-American Cooperative Research on Marine Mammals. Volume 1 - Pinnipeds: 55-59. National Oceanic and Atmospheric Administration, U.S. Department of Commerce, Silver Spring, Maryland.

Bull E.L. (2000). Seasonal and sexual differences in American marten diet in northeastern Oregon. Northwest Science 74: 186-191.

Burgin C.J., Colella J.P., Kahn P.L. \& Upham N.S. (2018). How many species of mammals are there? Journal of Mammalogy 99: 1-14. https://doi.org/10.1093/jmammal/gyx147

Bush B. (1995). Colitis in the dog. In Practice 17: 410-417. https://doi..org/10.1136/inpract.17.9.410

Bussotti F., Gravano E., Grossoni P. \& Tani C. (1998). Occurrence of tannins in leaves of beech trees (Fagus sylvatica) along an ecological gradient, detected by histochemical and ultrastructural analyses. The New Phytologist 138: 469-479.

ButLer J.R.A. \& DU ToIT J.T. (2002). Diet of free-ranging domestic dogs (Canis familiaris) in rural Zimbabwe: implications for wild scavengers on the periphery of wildlife reserves. Animal Conservation 5: 29-37. https://doi.org/10.1017/S136794300200104X

Buzzell B., Lance M.M. \& Acevedo-Gutiérrez A. (2014). Spatial and temporal variation in river otter (Lontra canadensis) diet and predation on rockfish (genus Sebastes) in the San Juan Islands, Washington. Aquatic Mammals 40: 150-161. https://doi.org/10.1578/AM.40.2.2014.150

CAMERON T.W.M. (1927). Observations on the life history of Ollulanus tricuspis Leuck., the stomach worm of the cat. Journal of Helminthology 5: 67-80. https://doi.org/10.1017/S0022149X00018630 
Campos C.B.D., Esteves C.F., Ferraz K.M.P.M.B., Crawshaw, Jr. P.G. \& Verdade L.M. (2007). Diet of free-ranging cats and dogs in a suburban and rural environment, south-eastern Brazil. Journal of Zoology 273: 14-20. https://doi.org/10.1111/j.1469-7998.2007.00291.x

CANNON M. (2013). Hair balls in cats: a normal nuisance or a sign that something is wrong? Journal of Feline Medicine and Surgery 15: 21-29. https://doi.org/10.1177/1098612X12470342

Casaux R., Baroni A. \& Carlini A. (1997). The diet of the Weddell seal Leptonychotes weddelli at Harmony Point, South Shetland Islands. Polar Biology 18: 371-375.

https://doi.org/10.1007/s003000050202

Casaux R., Juares M., Carlini A. \& CorbalánA. (2016). The diet of the Antarctic fur seal Arctocephalus gazella at the South Orkney Islands in ten consecutive years. Polar Biology 39: 1197-1206.

https://doi.org/10.1007/s00300-015-1836-3

Cashman J.L., Peirce M. \& Krausman P.R. (1992). Diets of mountain lions in southwestern Arizona. Southwestern Naturalist 37: 324-326.

Catling P.C. (1988). Similarities and contrasts in the diets of foxes, Vulpes vulpes, and cats, Felis catus, relative to fluctuating prey populations and drought. Australian Wildlife Research 15: 307-317. https://doi.org/10.1071/WR9880307

Cavallini P. \& Nel J.A.J. (1990). The feeding ecology of the Cape grey mongoose, Galerella pulverulenta (Wagner 1839) in a coastal area. African Journal of Ecology 28: 123-130.

https://doi.org/10.1111/j.1365-2028.1990.tb01144.x

Cavallini P. \& Serafini P. (1995). Winter diet of the small Indian mongoose, Herpestes auropunctatus, on an Adriatic Island. Journal of Mammalogy 76: 569-574. https://doi.org/10.2307/1382365

Chame M. (2003). Terrestrial mammal feces: a morphometric summary and description. Memórias do Instituto Oswaldo Cruz 98 (supplement I): 71-94. https://doi.org/10.1590/S0074-02762003000900014

Chernonosov A.A., Karpova E.A. \& Lyakh E.M. (2017). Identification of phenolic compounds in Myricaria bracteata leaves by high-performance liquid chromatography with a diode array detector and liquid chromatography with tandem mass spectrometry. Revista Brasileira de Farmacognosia 27: 576-579. https://doi.org/10.1016/j.bjp.2017.07.001

ChHangani A.K. (2002). Food and feeding of sloth bear (Melursus ursinus) in Aravalli Hills of Rajasthan, India. Tigerpaper 29: 1-4.

Chinchilla F.A. (1997). La dieta del jaguar (Panthera onca), el puma (Felis concolor) y el manigordo (Felis pardalis) (Carnivora: Felidae) en el Parque Nacional Corcovado, Costa Rica. Revista de Biologia Tropical 45: 1223-1229.

Choi H.G., Lee J.H., Park H.H. \& Sayegh F.A. (2009). Antioxidant and antimicrobial activity of Zostera marina L. extract. Algae 24: 179-184. https://doi.org/10.4490/ALGAE.2009.24.3.179

Christiansen P. (2008). Feeding ecology and morphology of the upper canines in bears (Carnivora: Ursidae). Journal of Morphology 269: 896-908.

Chua M.A., Sivasothi N. \& Meier R. (2016). Population density, spatiotemporal use and diet of the leopard cat (Prionailurus bengalensis) in a human-modified succession forest landscape of Singapore. Mammal Research 61: 99-108. https://doi.org/10.1007/s13364-015-0259-4

Chunang S.A. \& Lee L.L. (1997). Food habits of three carnivore species (Viverricula indica, Herpestes urva, and Melogale moschata) in Fushan Forest, northern Taiwan. Journal of Zoology 243: 71-79. https://doi.org/10.1111/j.1469-7998.1997.tb05757.x 
ĆIRoviĆ D. \& Penezić A. (2019). Importance of slaughter waste in winter diet of wolves (Canis lupus) in Serbia. North-western Journal of Zoology 15: 175-178.

Ćirović D., Penezić A., Milenković M. \& Paunović M. (2014). Winter diet composition of the golden jackal (Canis aureus L., 1758) in Serbia. Mammalian Biology 79: 132-137.

https://doi.org/10.1016/j.mambio.2013.11.003

Claridge A.W. \& May T.W. (1994). Mycophagy among Australian mammals. Australian Journal of Ecology 19: 251-275. https://doi.org/10.1111/j.1442-9993.1994.tb00489.x

Claridge A.W. \& Trappe J.M. (2005). Sporocarp mycophagy: nutritional, behavioural, evolutionaryand physiological aspects. In: Dighton J., White J.F. \& Oudemans P. (eds) The Fungal Community: Its Organization and Role in the Ecosystem, $3^{\text {rd }}$ ed.: 599-611. Taylor \& Francis, Boca Raton, Florida.

Clarke M.R. \& MacLeod N. (1982). Cephalopod remains in the stomachs of eight Weddell seals. British Antarctic Survey Bulletin 57: 33-40.

Cleary G.P., Corner L.A., O'KeefFe J. \& Marples N.M. (2009). The diet of the badger Meles meles in the Republic of Ireland. Mammalian Biology-Zeitschrift für Säugetierkunde 74: 438-447. https://doi.org/10.1016/j.mambio.2009.07.003

Clevenger A.P. (1993). Pine marten (Martes martes Linné, 1758) comparative feeding ecology in an island and mainland population of Spain. Zeitschrift fur Saugetierkunde 58: 212-224.

Clevenger A.P. (1995). Seasonality and relationships of food resource use of Martes martes, Genetta genetta and Felis catus in the Balearic Islands. Revue d'Ecologie 50: 109-131.

CLINE K.F. (1966). Diets for Siberian tigers Panthera tigris longipilis at Detroit Zoo. International Zoo Yearbook 6: 74-78. https://doi.org/10.1111/j.1748-1090.1966.tb01698.x

Colon C.P. \& Sugau J.B. (2012). Notes on the diet of the Malay civet (Viverra tangalunga) and other civets in logged and unlogged lowland dipterocarp rain forests in Sabah, Borneo. Malayan Nature Journal 64: 69-74.

ConDy P.R. (1981). Annual food consumption, and seasonal fluctuations in biomass of seals at Marion Island. Mammalia 45: 21-30. https://doi.org/10.1515/mamm.1981.45.1.21

Cook D.B. \& Hamilton W.J. (1944). The ecological relationships of red fox food in eastern New York. Ecology 25: 91-104. https://doi.org/10.2307/1930765

COOPER R.L. \& SKInNER J.D. (1979). Importance of termites in the diet of the aardwolf Proteles cristatus in South Africa. South Africa Journal of Zoology 14: 5-8.

https://doi.org/10.1080/02541858.1979.11447640

CoRlett R.T. (2017). Frugivory and seed dispersal by vertebrates in tropical and subtropical Asia: an update. Global Ecology and Conservation 11: 1-22. https://doi.org/10.1016/j.gecco.2017.04.007

CRABв W.D. (1941). Food habits of the prairie spotted skunk in southeastern Iowa. Journal of Mammalogy 22: 349-364. https://doi.org/10.2307/1374928

Cronk N. \& Pillay N. (2019). Dietary overlap of two sympatric African mongoose species in an urban environment. Mammalia 83: 428-438. https://doi.org/10.1515/mammalia-2018-0113

CulPePer N. (1666). The English Physitian Enlarged. John Streater, London.

CZERniK M., KowalczyK R. \& ZaLewski A. (2016). Spatio-temporal variation of predator diet in a rural habitat: stone martens in the villages of Białowieża forest. Mammal Research 61: 187-196.

https://doi.org/10.1007/s13364-016-0273-1 
Daneri G.A. \& Coria N.R. (1992). The diet of Antarctic fur seals, Arctocephalus gazella, during the summer-autumn period at Mossman Peninsula, Laurie Island (South Orkneys). Polar Biology 11: 565566. https://doi.org/10.1007/BF00237949

Daneri G.A., Carlini A.R., Harrington A., Balboni L. \& Hernandez C.M. (2008). Interannual variation in the diet of non-breeding male Antarctic fur seals, Arctocephalus gazella, at Isla 25 de Mayo/ King George Island. Polar Biology 31: 1365-1372. https://doi.org/10.1007/s00300-008-0475-3

DAYANANDAN P. (1983). Localization of silica and calcium carbonate in plants. Scanning Electron Microscopy 3: 1519-1524.

de Araujo C.C.M. (2008). Hábito alimentar do cachorro-do-mato, Cerdocyon thous (Carnivora: Canidae), numa área de restinga, na ilha de cananéia, Estado de São Paulo. Bachelors degree. Universidade Federal do Paraná, Paraná, Brazil.

DE LA Torre J.A. \& DE LA Riva G. (2009). Food habits of pumas (Puma concolor) in a semiarid region of central Mexico. Mastozoología Neotropical 16: 211-216.

De LuCA D.W. \& Rovero F. (2006). First records in Tanzania of the vulnerable Jackson's mongoose Bdeogale jacksoni (Herpestidae). Oryx 40: 468-471. https://doi.org/10.1017/S0030605306001396

Deal M.S., HaY M.E., Wilson D. \& Fenical W. (2003). Galactolipids rather than phlorotannins as herbivore deterrents in the brown seaweed Fucus vesiculosus. Oecologia 136: 107-114. https://doi.org/10.1007/s00442-003-1242-3

Delibes M. (1978). Feeding habits of the stone marten, Martes foina (Erxleben, 1777), in northern Burgos, Spain. Zeitschrift für Säugetierkunde 43: 282-288.

Delibes M., Aymerich M. \& Cuesta L. (1984). Feeding habits of the Egyptian mongoose or ichneumon in Spain. Acta Theriologica 29: 205-218.

de Villa Meza A., Meyer E.M. \& González C.A.L. (2002). Ocelot (Leopardus pardalis) food habits in a tropical deciduous forest of Jalisco, Mexico. The American Midland Naturalist 148: 146-155. https://doi.org/10.1674/0003-0031(2002)148[0146:OLPFHI]2.0.CO;2

Devkota B.P., Silwal T. \& Kolejka J. (2013). Prey density and diet of snow leopard (Uncia uncia) in Shey Phoksundo National Park, Nepal. Applied Ecology and Environmental Sciences 1: 55-60. https://doi.org/10.12691/aees-1-4-4

Dixon J. (1925). Food predilections of predatory and fur-bearing mammals. Journal of Mammalogy 6: 34-46. https://doi.org/10.2307/1373468

Donadelli R.A. (2019). Effects of Miscanthus grass as a Fiber Source in Pet Diets on Extrusion Processing and Diet Utilization by Dogs and Cats. Ph.D. dissertation. Kansas State University, Manhattan, Kansas.

Drouilly M., NatTrass N. \& O'RIAIN M.J. (2018). Dietary niche relationships among predators on farmland and a protected area. The Journal of Wildlife Management 82: 507-518. https://doi.org/10.1002/jwmg.21407

Dubay S.A., Hayward G.D. \& Martinez del Rio C. (2008). Nutritional value and diet preference of arboreal lichens and hypogeous fungi for small mammals in the Rocky Mountains. Canadian Journal of Zoology 86: 851-862. https://doi.org/10.1139/Z08-054

Dubinin E.A. (2012). The diet of Mustela erminea L. in the Magadan Oblast. Contemporary Problems of Ecology 5: 110-114. https://doi.org/10.1134/S1995425512010158

Dudley J.P. (1892). Homo et Canis: or, the Autobiography of Old Cato and Some Account of His Race, vol. 2. The Republic Publishing Company, Chicago and Cincinnati. 
Duncan A. (1956). Notes on the food and parasites of grey seals, Halichoerus grypus (Fabricius), from the Isle of Man. Proceedings of the Zoological Society of London 126: 635-644.

https://doi.org/10.1111/j.1096-3642.1956.tb00455.x

Durbin J., Funk S.M., Hawkins F., Hills D.M., Jenkins P.D., MoncriefF C.B. \& Ralainasolo F.B. (2010). Investigations into the status of a new taxon of Salanoia (Mammalia: Carnivora: Eupleridae) from the marshes of Lac Alaotra, Madagascar. Systematics and Biodiversity 8: 341-355. https://doi.org/10.1080/14772001003756751

DyкYY I. (2009). The feeding peculiarities of the antarctic seals in the region of the archipelago of Argentina islands. Український антарктичний журнал 8: 311-319.

EADIE W.R. (1943). Food of the red fox in southern New Hampshire. The Journal of Wildlife Management 7: 74-77.

Eddine A., Mostefai N., De Smet K., Klees D., Ansorge H., Karssene Y., Nowak C. \& van der Leer P. (2017). Diet composition of a newly recognized canid species, the African golden wolf (Canis anthus), in northern Algeria. Annals Botanici Fennici 54: 347-356. https://doi.org/10.5735/086.054.0506

Ellis R.J. (1951). Food Habits and Control of Coyotes in Northcentral Oklahoma. M.S. Thesis. Oklahoma State University, Stillwater, Oklahoma.

Ellis R.J. \& Schemnitz S.D. (1958). Some foods used by coyotes and bobcats in Cimarron County, Oklahoma 1945 through 1956. Proceedings of the Oklahoma Academy of Sciences 38: 180-185.

Elmeros M., Mikkelsen D.M.G., Nørgaard L.S., Pertoldi C., Jensen T.H. \& Chriél M. (2018). The diet of feral raccoon dog (Nyctereutes procyonoides) and native badger (Meles meles) and red fox (Vulpes vulpes) in Denmark. Mammal Research 63: 405-413.

https://doi.org/10.1007/s13364-018-0372-2

Elmhagen B., TANnerfeldt M. \& AngerbJörn A. (2002). Food-niche overlap between arctic foxes and red foxes. Canadian Journal of Zoology 80: 1274-1285. https://doi.org/10.1139/z02-108

Emmons L.H. (1987). Comparative feeding ecology of felids in a neotropical rainforest. Behavioral Ecology and Sociobiology 20: 271-283.

ENGEL T.R. (1998). Seeds on the roundabout - tropical forest regeneration by Genetta rubiginosa. Small Carnivore Conservation 19: 13-20.

ERrington P.L. (1935). Food habits of mid-west foxes. Journal of Mammalogy 16: 192-200. https://doi.org/10.2307/1374445

ERRINGTON P.L. (1937). Summer food habits of the badger in northwestern Iowa. Journal of Mammalogy 18: 213-216. https://doi.org/10.2307/1374470

Fabregas M.C., Garcés-Narro C., Bertschinger H. \& Koehler G. (2016). Carcass utilization by tigers: implications for calculating prey requirements. Journal of Zoology 301: 141-149.

https://doi.org/10.1111/jzo.12403

FACCOLI M. \& SCHLYTER F. (2007). Conifer phenolic resistance markers are bark beetle antifeedant semiochemicals. Agricultural and Forest Entomology 9: 237-245.

https://doi.org/10.1111/j.1461-9563.2007.00339.x

Fajardo U., Cossíos D. \& Pacheco V. (2014). Dieta de Leopardus colocolo (Carnivora: Felidae) en la Reserva Nacional de Junín, Junín, Perú. Revista Peruana de Biología 21: 61-70.

https://doi.org/10.15381/rpb.v21i1.8248 
Farrell L.E., Roman J. \& Sunquist M.E. (2000). Dietary separation of sympatric carnivores identified by molecular analysis of scats. Molecular Ecology 9: 1583-1590.

https://doi.org/10.1046/j.1365-294x.2000.01037.x

Faure J.P.B., Holmes N.J., Watson L.H. \& Hill R.A. (2019). Brown hyaena (Parahyaena brunnea) diet composition from Zingela Game Reserve, Limpopo Province, South Africa. African Zoology 54: $119-124$.

FAY F.H. \& STEPHENSON R.O. (1989). Annual, seasonal, and habitat-related variation in feeding habits of the arctic fox (Alopex lagopus) on St. Lawrence Island, Bering Sea. Canadian Journal of Zoology 67: 1986-1994. https://doi.org/10.1139/z89-283

Fenn E. (1790). The Rational Dame; or, Hints towards Supplying Prattle for Children. John Marshall and Co. at No. 4, London.

Ferreira G.A., Nakano-Oliveira E., Genaro G. \& Lacerda-Chaves A.K. (2013). Diet of the coati Nasua nasua (Carnivora: Procyonidae) in an area of woodland inserted in an urban environment in Brazil. Revista Chilena de Historia Natural 86: 95-102.

Fowler A., Koutsioni Y. \& Sommer V. (2007). Leaf-swallowing in Nigerian chimpanzees: evidence for assumed self-medication. Primates 48: 73-76. https://doi.org/10.1007/s10329-006-0001-6

Francis G.R. (1958). Ecological Studies of Marten (Martes americana) in Algonquin Park Ontario. M.S. Thesis. University of British Columbia, Vancouver, British Columbia.

Fritts S.H. \& SEALANDER J.A. (1978). Diets of bobcats in Arkansas with special reference to age and sex differences. Journal of Wildlife Management 42: 533-539. https://doi.org/10.2307/3800815

Furusaka S., Kozakai C., Nemoto Y., Umemura Y., Naganuma T., Yamazaki K. \& Koike S. (2017). The selection by the Asiatic black bear (Ursus thibetanus) of spring plant food items according to their nutritional values. ZooKeys 672: 121-133. https://doi.org/10.3897/zookeys.672.10078

Gade-Jørgensen I. \& StagegaARd R. (2000). Diet composition of wolves Canis lupus in east-central Finland. Acta Theriologica 45: 537-547.

Gainsbury A.M., Tallowin O.J. \& Meiri S. (2018). An updated global data set for diet preferences in terrestrial mammals: testing the validity of extrapolation. Mammal Review 48: 160-167. https://doi.org/10.1111/mam.12119

Gamenara D., Pandolfi E., Saldaña J., Domínguez L., Martínez M.M. \& Seoane G. (2001). Nematocidal activity of natural polyphenols from bryophytes and their derivatives. Arzneimittelforschung 51: 506-510.

GARCía J.P.T. (1998). Estudio del nicho alimenticio de los mamíferos depredadores del orden Carnivora en La Sierra del Carmen, Noroeste de Coahuila, México. M.S. Thesis. Universidad Autónoma de Nuevo León, Nuevo León, Mexico.

GARCía-RANGel S. (2012). Andean bear Tremarctos ornatus natural history and conservation. Mammal Review 42: 85-119.

García-Villalba R., EsPín J.C., TOMás-BarberánF.A. \& Rocha-GuZmán N.E. (2017). Comprehensive characterization by LC-DAD-MS/MS of the phenolic composition of seven Quercus leaf teas. Journal of Food Composition and Analysis 63: 38-46. https://doi.org/10.1016/j.jfca.2017.07.034

Garla R.C., Setz E.Z.F. \& GobBi N. (2001). Jaguar (Panthera onca) food habits in Atlantic Rain Forest of southeastern Brazil. Biotropica 33: 691-696. https://doi.org/10.1111/j.1744-7429.2001.tb00226.x

GefFen E., Hefner R., MacDonald D.W. \& UcKo M. (1992). Diet and foraging behavior of Blanford's foxes, Vulpes cana, in Israel. Journal of Mammalogy 73: 395-402. https://doi.org/10.2307/1382074 
George-Nascimento M., Bustamante R. \& Oyarzun C. (1985). Feeding ecology of the South American sea lion Otaria flavescens: Food contents and food selectivity. Marine Ecology - Progress Series 21: 135-143.

Giannatos G., Karypidou A., Legakis A. \& Polymeni R. (2010). Golden jackal (Canis aureus L.) diet in Southern Greece. Mammalian Biology 75: 227-232. https://doi.org/10.1016/j.mambio.2009.03.003

Giordana C., Lyra-Jorge M.C., Miotto R.A. \& Pivello V.R. (2018). Food habits of three carnivores in a mosaic landscape of São Paulo state, Brazil. European Journal of Wildlife Research 64: 15. https://doi.org/10.1007/s10344-018-1172-3

GIPSON P.S. (1974). Food habits of coyotes in Arkansas. Journal of Wildlife Management 38: 848-853. https://doi.org/10.2307/3800055

Glen A.S. \& Dickman C.R. (2006). Diet of the spotted-tailed quoll (Dasyurus maculatus) in eastern Australia: effects of season, sex and size. Journal of Zoology 269: 241-248.

https://doi.org/10.1111/j.1469-7998.2006.00046.x

Glen A.S. \& Dickman C.R. (2008). Niche overlap between marsupial and eutherian carnivores: does competition threaten the endangered spotted-tailed quoll? Journal of applied Ecology 45: 700-707. https://doi.org/10.1111/j.1365-2664.2007.01449.x

Glen A.S., De Tores P.J., Sutherland D.R. \& Morris K.D. (2009). Interactions between chuditch (Dasyurus geoffroii) and introduced predators: a review. Australian Journal of Zoology 57: 347-356. https://doi.org/10.1071/ZO09041

Goldyn B., Hromada M., Surmacki A. \& Tryjanowski P. (2003). Habitat use and diet of the red fox Vulpes vulpes in an agricultural landscape in Poland. Zeitschrift für Jagdwissenschaft 49: 191-200. https://doi.org/10.1007/BF02189737

Golightly R.T., Penland T.F., Zielinski W.J. \& Higley J.M. (2006). Fisher Diet in the Klamath/ North Coast Bioregion. Final report to U.S. Fish \& Wildlife Service. Humboldt State University, Arcata, California.

Gomes D.J. ,Wierzbowska I.A., Bevanger K., O’Mahony D.T., \& Rola K. (2020). Diet of the European badgers (Meles meles) in urban and rural areas of Norway. European Journal of Wildlife Research 66: 7. https://doi.org/10.1007/s10344-019-1347-6

Gómez-Ortiz Y., Fajardo V., Monroy-Vilchis O., Mendoza G.D. \& Urios V. (2011). Is food quality important for carnivores? The case of Puma concolor. Animal Biology 61: 277-288.

https://doi.org/10.1163/157075511X584227

Gordon C.C. (1986). Winter food habits of the pine marten in Colorado. The Great Basin Naturalist 46: $166-168$.

Gori M., Carpaneto G.M. \& Ottino P. (2003). Spatial distribution and diet of the Neotropical otter Lontra longicaudis in the Ibera Lake (northern Argentina). Acta Theriologica 48: 495-504.

https://doi.org/10.1007/BF03192495

Gorman M.L. (1975). The diet of feral Herpestes auropunctatus (Carnivora: Viverridae) in the Fijian Islands. Journal of Zoology 175: 273-278. https://doi.org/10.1111/j.1469-7998.1975.tb01401.x

Gormezano L.J. \& Rockwell R.F. (2013). What to eat now? Shifts in polar bear diet during the ice-free season in western Hudson Bay. Ecology and Evolution 3: 3509-3523. https://doi.org/10.1002/ece3.740

Gourvelou E., Papageorgiou N. \& Neophytou C. (2000). Diet of the otter Lutra lutra in lake Kerkini and stream Milli-Aggistro, Greece. Acta Theriologica 45: 35-44. 
Grassman L.I., Tewes M.E., Silvy N.J. \& Kreetiyutanont K. (2005). Spatial organization and diet of the leopard cat (Prionailurus bengalensis) in north-central Thailand. Journal of Zoology 266: 45-54. https://doi.org/10.1017/S095283690500659X

Green K. \& Burton H.R. (1987). Seasonal and geographical variation in the food of Weddell seals, Leptonychotes weddelii, in Antarctica. Australian Wildlife Research 14: 475-489.

https://doi.org/10.1071/WR9870475

GreEN R.H. (1967). Notes on the devil (Sarcophilus harrisi) and the quoll (Dasyurus viverrinus) in north-eastern Tasmania. Records of the Queen Victoria Museum 27: 1-13.

GRENFELl W.E. \& FASENFEST M. (1979). Winter food habits of fisher (Martes pennanti) in northwestern California. California Fish and Game 65: 186-189.

GuY P. (1977). A note on the food of the civet Viverra civetta (Schreber), in the Sengwa Wildlife Research Area, Rhodesia. South African Journal of Wildlife Research 7: 87-88.

Habtamu T., Bekele A., Ahmed R., Gadisa T., Birlie B., Tolemariam T. \& Belay B. (2017). Diets of the African civet Civettictis civetta (Schreber, 1778) in selected coffee forest habitat, south-western Ethiopia. African Journal of Ecology 55: 573-579. https://doi.org/10.1111/aje.12390

Haight C.P. (1937). Some Observations on the Predator-Prey Complex in the Gallatin Valley. M.Sc. Thesis. Montana State College, Bozeman, Montana.

Hall M.C. (1919). Practical methods of treatment for worm infestation. Journal of the American Veterinary Medical Association 55: 24-45.

Halloran A.F. \& Glass B.P. (1959). The carnivores and ungulates of the Wichita Mountains Wildlife Refuge, Oklahoma. Journal of Mammalogoy 40: 360-370. https://doi.org/10.2307/1376560

HämÄläinen A., Broadley K., Droghini A., Haines J.A., Lamb C.T., Boutin S. \& Gilbert S. (2017). The ecological significance of secondary seed dispersal by carnivores. Ecosphere 8: e01685.

HAmilton Jr. W.J. \& Hunter R.P. (1939). Fall and winter food habits of Vermont bobcats. The Journal of Wildlife Management 3: 99-103. https://doi.org/10.2307/3796351

Hamilton Jr. W.J., Hosley N.W. \& MacGregor A.E. (1937). Late summer and early fall foods of the red fox in central Massachusetts. Journal of Mammology 18: 366-367.

https://doi.org/10.1093/jmammal/18.3.366

Hanson K. \& Moen R. (2008). Diet of Canada Lynx in Minnesota estimated from Scat Analysis. NRRI Technical Report no. NRRI/TR-2008-13.

HART B.L. (2008). Why do dogs and cats eat grass? Veterinary Medicine December 2008: 648-649.

HART B.L. (2011). Behavioural defences in animals against pathogens and parasites: parallels with the pillars of medicine in humans. Philosophical Transactions of the Royal Society B: Biological Sciences 366: 3406-3417. https://doi.org/10.1098/rstb.2011.0092

Hart B.L. \& Hart L.A. (2013). Your Ideal Cat. Purdue University Press, West Lafayette, Indiana.

HART B.L. \& Hart L.A. (2018). How mammals stay healthy in nature: the evolution of behaviours to avoid parasites and pathogens. Philosophical Transactions of the Royal Society B, Biological Sciences 373: 20170205. https://doi.org/10.1098/rstb.2017.0205

Hart B.L., Hart L.A. \& Thigpen A.P. (2019). Characterization of plant eating in cats. In: Newberry R.C. \& BraAstad B.O. (eds) Proceedings of the $53^{\text {rd }}$ Congress of the ISAE: 106. Wageningen Academic Publishers, Netherlands. 
Hartová-Nentvichová M., Š́́LEK M., Červeny \& KoubeK P. (2010). Variation in the diet of the red fox (Vulpes vulpes) in mountain habitats: Effects of altitude and season. Mammalian Biology 75: 334-340. https://doi.org/10.1016/j.mambio.2009.09.003

HATFieLD D.M. (1939). Winter food habits of foxes in Minnesota. Journal of Mammalogy 20: 202-206. https://doi.org/10.2307/1374378

Haug T., Nilssen K. T. \& Lindblom L. (2000). First independent feeding of harp seal (Phoca groenlandica) and hooded seal (Cystophora cristata) pups in the Greenland Sea. NAMMCO Scientific Publications 2: 29-39. https://doi.org/10.7557/3.2969_

HeLlDiN J.-O. (1999). Diet, body condition, and reproduction of Eurasian pine martens Martes martes during cycles in microtine density. Ecography 22: 324-336.

https://doi.org/10.1111/j.1600-0587.1999.tb00508.x

Helldin J.O. (2000). Seasonal diet of pine marten Martes martes in southern boreal Sweden. Acta Theriologica 45: 409-420.

Henschel J.R. \& Skinner J.D. (1990). The diet of the spotted hyaenas Crocuta crocuta in Kruger National Park. African Journal of Ecology 28: 69-82.

https://doi.org/10.1111/j.1365-2028.1990.tb01138.x

Herrera C.M. (1989). Frugivory and seed dispersal by carnivorous mammals, and associated fruit characteristics, in undisturbed Mediterranean habitats. Oikos 55: 250-262.

https://doi.org/10.2307/3565429

Hidalgo-Mihart M.G., Cantú-Salazar L., LóPez-González C.A., Martínez-Meyer E. \& GonzÁlez-Romero A. (2001). Coyote (Canis latrans) food habits in a tropical deciduous forest of western Mexico. The American Midland Naturalist 146: 210-216.

HiLl E.L. (1979). The ecology of the timber wolf (Canis lupus Linn.) in southern Manitoba - wilderness, recreational and agricultural aspects. M.Sc. Thesis. University of Manitoba, Winnipeg, Manitoba.

Hipólito D., Santos-Reis M. \& Rosalino L.M. (2016). European badger (Meles meles) diet in an agroforestry and cattle ranching area of Central-West Portugal. Wildlife Biology in Practice 12: 1-13.

Hisano M., Raichev E.G., Peeva S., Tsunoda H., Newman C., Masuda R., Georgiev D.M. \& KANEKO Y. (2016). Comparing the summer diet of stone martens (Martes foina) in urban and natural habitats in Central Bulgaria. Ethology Ecology \& Evolution 28: 295-311.

https://doi.org/10.1080/03949370.2015.1048829

Hiscocks K. \& Perrin M.R. (1987). Feeding observations and diet of black-backed jackals in an arid coastal environment. South African Journal of Wildlife Research 17: 55-58.

Huelset A. M., Andersen M., Gjertz I., Lydersen C. \& Gulliksen B. (1999). Feeding habits of bearded seals (Erignathus barbatus) from the Svalbard area, Norway. Polar Biology 21: 186-193. https://doi.org/10.1007/s003000050351

Hockman J.G. \& Chapman J.A. (1983). Comparative feeding habits of red foxes (Vulpes vulpes) and gray foxes (Urocyon cinereoargenteus) in Maryland. The American Midland Naturalist 110: 276-285.

Holle D.G. (1973). Diet and General Availability of Prey of the Coyote (Canis latrans) at the Wichita Mountains National Wildlife Refuge, Oklahoma. Master Thesis. Oklahoma State University, Stillwater, Oklahoma.

Hopkins D.D. \& Forbes R.B. (1980). Dietary patterns of the Virginia opossum in an urban environment. The Murrelet 61: 20-30. https://doi.org/10.2307/3536187 
Hoppe-Dominik B. (1988). Grass-eating leopards: Wolves turned into sheep? Naturwissenschaften 75: 49-50. https://doi.org/10.1007/BF00367444

Houston D.C., Mee A. \& McGrady M. (2007). Why do condors and vultures eat junk?: the implications for conservation. Journal of Raptor Research 41: 235-239.

https://doi.org/10.3356/0892-1016(2007)41[235:WDCAVE]2.0.CO;2

HufFMAn M.A. (1997). Current evidence for self-medication in primates: a multidisciplinary perspective. American Journal of Physical Anthropology 104: 171-200.

HuffMan M.A. \& CATON J.M. (2001). Self-induced increase of gut motility and the control of parasitic infections in wild chimpanzees. International Journal of Primatology 22: 329-346.

https://doi.org/10.1023/A:1010734310002

Huffman M.A., Page J.E., Sukhdeo M.V., Gotoh S., Kalunde M.S., Chandrasiri T. \& Towers G.N. (1996). Leaf-swallowing by chimpanzees: a behavioral adaptation for the control of strongyle nematode infections. International Journal of Primatology 17: 475-503. https://doi.org/10.1007/BF02735188

HuIDEKoper R.S. (1895). A Guide to the Classification and Varieties of Cats and a Short Treatise upon their Care, Diseases, and Treatment. D. Appleton and Company, New York, New York.

Humphries B.D., RAMESH T. \& Downs C.T. (2015). Diet of black-backed jackals (Canis mesomelas) on farmlands in the KwaZulu-Natal Midlands, South Africa. Mammalia 80: 405-412.

https://oi.org/10.1515/mammalia-2014-0103

HusSAIN S.A. (2013). Activity pattern, behavioural activity and interspecific interaction of smoothcoated otter (Lutrogale perspicillata) in National Chambal Sanctuary, India. IUCN Otter Specialist Group Bulletin 30: 5-17.

Hutton A.F. (1944). Feeding habits of the Nilgiri marten. Journal of the Bombay Natural History Society 48: 374-375.

IHL C. \& BARBOza P.S. (2007). Nutritional value of moss for arctic ruminants: a test with muskoxen. The Journal of Wildlife Management 71: 752-758. https://doi.org/10.2193/2005-745

InCORVAIA G. (2005). Etude des facteurs potentiellement limitant de la répartition des fennecs, Fennecus zerda, dans le sud-tunisien. Ph.D. Thesis. Université Claude-Bernard, Lyon, France.

Iriarte J.A., Jimenez J.E., Contreras L.C. \& Jaksić F.M. (1989). Small-mammal availability and consumption by the fox Dusicyon culpaeus in central Chilean scrublands. Journal of Mammalogy 70: 641-645. https://doi.org/10.2307/1381441

Iriarte J.A., Johnson W.E. \& FrankLIN W.L. (1991). Feeding ecology of the Patagonia puma in southernmost Chile. Revista Chilena de Historia Natural 64: 145-156.

Iwama M., Yamazaki K., Matsuyama M., Hoshino Y., Hisano M., Newman C. \& Kaneko Y. (2017). Masked palm civet Paguma larvata summer diet differs between sexes in a suburban area of central Japan. Mammal Study 42: 185-190. https://doi.org/10.3106/041.042.0301

Jackson S.M., Groves C.P., Fleming P.J., Aplin K.P., Eldridge M.D., GonZalez A. \& Helgen K.M. (2017). The wayward dog: is the Australian native dog or dingo a distinct species? Zootaxa 4317: 201224. https://doi.org/10.11646/zootaxa.4317.2.1

JÁcomo A.T. DE A., Silveira L. \& Diniz-Filho J.A.F. (2004). Niche separation between the maned wolf (Chrysocyon brachyurus), the crab-eating fox (Dusicyon thous) and the hoary fox (Dusicyon vetulus) in central Brazil. Journal of Zoology 262: 99-106. https://doi.org/10.1017/S0952836903004473

JAKsić F.M., YÁÑEZ J.L. \& RAU J.R. (1983). Trophic relations of the southernmost populations of Dusicyon in Chile. Journal of Mammology 64: 693-697. https://doi.org/10.2307/1380529 
JĘDRZEJEWSKi W. \& JĘDRZEJEWSKI B. (1992). Foraging and diet of the red fox Vulpes vulpes in relation to variable food resources in Białowieża National Park, Poland. Ecography 15: 212-220.

https://doi.org/10.1111/j.1600-0587.1992.tb00027.x

Jobin A., Molinari P. \& Breitenmoser U. (2000). Prey spectrum, prey preference and consumption rates of Eurasian lynx in the Swiss Jura Mountains. Acta Theriologica 45: 243-252.

Johnsingh A.J.T. (1978). Some aspects of the ecology and behaviour of the Indian fox - Vulpes bengalensis (Shaw). The Journal of the Bombay Natural History Society 75: 397-405.

JoHnsingh A.J.T. (1983). Large mammalian prey - predators in Bandipur. The Journal of the Bombay Natural History Society 80: 1-57.

Johnson K.G., Wei W., ReId D.G. \& Jinchu H. (1993). Food habits of Asiatic leopards (Panthera pardus fusea) in Wolong Reserve, Sichuan, China. Journal of Mammalogy 74: 646-650.

https://doi.org/10.2307/1382285

JuAREZ K. M. \& MARINHo-Filho J. (2002). Diet, habitat use, and home ranges of sympatric canids in Central Brazil. Journal of Mammalogy 83: 925-933.

https://doi.org/10.1644/1545-1542(2002)083<0925:DHUAHR>2.0.CO;2

Julkunen-TittTo R. (1985). Phenolic constituents in the leaves of northern willows: methods for the analysis of certain phenolics. Journal of Agricultural and Food Chemistry 33: 213-217.

https://doi.org/10.1021/jf00062a013

Jumabay-Uulu K., Wegge P., Mishra C. \& Sharma K. (2014). Large carnivores and low diversity of optimal prey: a comparison of the diets of snow leopards Panthera uncia and wolves Canis lupus in Sarychat-Ertash Reserve in Kyrgyzstan. Oryx 48: 529-535. https://doi.org/10.1017/S0030605313000306

KALlE R., RAMESH T., SANKAR K. \& QURESHI Q. (2012). Diet of mongoose in mudumalai tiger reserve, southern India. Journal of Scientific Transactions in Environment and Technovation 6: 44-51.

Kang B.T., Jung D.I., Yoo J.H., Park C., Woo E.J. \& Park H.M. (2007). A high fiber diet responsive case in a poodle dog with long-term plant eating behavior. Journal of Veterinary Medical Science 69: 779-782. https://doi.org/10.1292/jvms.69.779

KAPEL C.M. (1999). Diet of arctic foxes (Alopex lagopus) in Greenland. Arctic 52: 289-293.

Kapfer P.M., Streby H.M., Gurung B., Simcharoen A., McDougal C.C. \& Smith J.L. (2011). Fine-scale spatio-temporal variation in tiger Panthera tigris diet: effect of study duration and extent on estimates of tiger diet in Chitwan National Park, Nepal. Wildlife Biology 17: 277-286.

https://doi.org/10.2981/10-127

Katiki L.M., Chagas A.C.S., Bizzo H.R., Ferreira J.F.S. \& Amarante A.F.T.D. (2011). Anthelmintic activity of Cymbopogon martinii, Cymbopogon schoenanthus and Mentha piperita essential oils evaluated in four different in vitro tests. Veterinary Parasitology 183: 103-108.

https://doi.org/10.1016/j.vetpar.2011.07.001

Kaufman P.B., Dayanandan P., Takeoka Y., Bigelow W.C., Jones J.D. \& Iler R. (1981). Silica in shoots of higher plants. In: Simpson T.L. \& Volcani B.E. (eds) Silicon and Siliceous Structures in Biological Systems: 409-449. Springer-Verlag, New York, New York.

KaYs R.W. (1999). Food preferences of kinkajous (Potos flavus): A frugivorous carnivore. Journal of Mammalogy 80: 589-599. https://doi.org/10.2307/1383303

Keeling C.I. \& Bohlmann J. (2006). Genes, enzymes and chemicals of terpenoid diversity in the constitutive and induced defence of conifers against insects and pathogens. New Phytologist 170: 657675. https://doi.org/10.1111/j.1469-8137.2006.01716.x 
Kelly E.C., Cypher B.L. \& Germano D.J. (2019). Exploitative competition between desert kit foxes and coyotes in the Mojave Desert. Pacific Conservation Biology 26: 57-66. https://doi.org/10.1071/PC19007

Khan K.A., Khan J.A. \& Mohan N. (2017). Winter food habits of the golden jackal Canis aureus (Mammalia: Carnivora: Canidae) in Patina Bird Sanctuary, Uttar Pradesh, India. Journal of Threatened Taxa 9: 10656-10661. https://doi.org/10.11609/jott.3301.9.9.10656-10661

Khan K.A., Khan J.A., Ahmad K. \& Mohan N. (2019). Winter food habits of the common palm civet Paradoxurus hermaphroditus (Mammalia: Carnivora: Viverridae) in Patna Bird Sanctuary, India. Journal of Threatened Taxa 11: 14490-14995.

Khan M.M.H. (2008). Prey selection by tigers Panthera tigris (Linnaeus 1758) in the Sundarbans East Wildlife Sanctuary of Bangladesh. The Journal of the Bombay Historical Society 105: 255-263.

Kidawa D. \& KowalczyK R. (2011). The effects of sex, age, season and habitat on diet of the red fox Vulpes vulpes in northeastern Poland. Acta Theriologica 56: 209-218.

https://doi.org/10.1007/s13364-011-0031-3

KirKPATRICK R.D. \& RAUZON M.J. (1986). Foods of feral cats Felis catus on Jarvis and Howland Islands, central Pacific Ocean. Biotropica 18: 72-75. https://doi.org/10.2307/2388365

Kissling W.D., Dalby L., Fløjgaard C., Lenoir J., Sandel B., Sandom C., Trøjelsgaard K. \& SvENNING J.C. (2014). Establishing macroecological trait datasets: digitalization, extrapolation, and validation of diet preferences in terrestrial mammals worldwide. Ecology and Evolution 4: 2913-2930. https://doi.org/10.1002/ece3.1136

Kistchinski A.A. (1972). Life history of the brown bear (Ursus arctos L.) in north-east Siberia. Bears: Their Biology and Management 2: 67-73. https://doi.org/10.2307/3872570

Kitchen A.M., Gese E.M. \& SChauster E.R. (1999). Resource partitioning between coyotes and swift foxes: space, time, and diet. Canadian Journal of Zoology 77: 1645-1656.

https://doi.org/10.1139/z99-143

Klare U., Kamler J.F. \& Macdonald D.W. (2011a). A comparison and critique of different scatanalysis methods for determining carnivore diet. Mammal Review 41: 294-312.

https://doi.org/10.1111/j.1365-2907.2011.00183.x

Klare U., Kamler J.F. \& Macdonald D.W. (2011b). The bat-eared fox: A dietary specialist? Mammalian Biology 76: 646-650. https://doi.org/10.1016/j.mambio.2011.06.005

KoK O.B. \& Nel J.A.J. (1992). Diet of the bat-eared fox in the Orange Free State and northern Cape Province. South African Journal of Wildlife Research 22: 36-39.

Krawczyk A.J., SkierczyŃski M. \& Tryjanowski P. (2011). Diet of the Eurasian otter Lutra lutra on small watercourses in Western Poland. Mammalia 75: 207-210. https://doi.org/10.1515/mamm.2011.005

Krishnakumar B.M., Nagarajan R., Selvan K.M. \& Thinesh T. (2019). Dhole: snake in the diet of Cuon alpinus (Pallas, 1811) in Kalakad-Mundanthurai Tiger Reserve, Tamil Nadu. Mammal Tales \#9. Zoo's Print 34: 38-40.

Krishnamani R. \& Mahaney W.C. (2000). Geophagy among primates: adaptive significance and ecological consequences. Animal Behaviour 59: 899-915. https://doi.org/10.1006/anbe.1999.1376

Krofel M., Huber D. \& Kos I. (2011). Diet of Eurasian lynx Lynx lynx in the northern Dinaric Mountains (Slovenia and Croatia). Acta Theriologica 56: 315-322. https://doi.org/10.1007/s13364-011-0032-2

KRUUK H. \& SANDS W.A. (1972). The aardwolf(Proteles cristatus Sparrman) 1783 as predator of termites. East African Wildlife Journal 10: 211-227. https://doi.org/10.1111/j.1365-2028.1972.tb00728.x 
Ksouri R., Falleh H., Megdiche W., Trabelsi N., Mhamdi B., Chaieb K., Bakrouf A., Magné C. \& ABDELLY C. (2009). Antioxidant and antimicrobial activities of the edible medicinal halophyte Tamarix gallica L. and related polyphenolic constituents. Food and Chemical Toxicology 47: 2083-2091. https://doi.org/10.1016/j.fct.2009.05.040

KUMARI Y.S. (2015). Evaluation of in vitro anthelmintic activity of bryophyte Pottia lanceolata. International Journal of Innovative Science, Engineering \& Technology 3: 418-423.

KuYT E. (1969). Feeding Ecology of Wolves on Barren-ground Caribou Range in the Northwest Territories. M.A. Thesis. University of Saskatchewan, Saskatoon, Saskatchewan.

Labansen A.L., Lydersen C., Haug T. \& Kovacs K.M. (2007). Spring diet of ringed seals (Phoca hispida) from northwestern Spitsbergen, Norway. ICES Journal of Marine Science 64: 1246-1256. https://doi.org/10.1093/icesjms/fsm090

LACK A. (1977). Genets feeding on nectar from Maranthes polyandra in Northern Ghana. East African Wildlife Journal 15: 233-234.

LANNING F.C. (1961). Calcite in Lesquerella ovalifolia trichomes. Science 133: 380. https://doi.org/10.1126/science.133.3450.380

Lanning F.C. \& Eleuterius L.N. (1989). Silica deposition in some C3 and C4 species of grasses, sedges and composites in the USA. Annals of Botany 64: 395-410.

https://doi.org/10.1093/oxfordjournals.aob.a087858

Lanning F.C., Ponnaiya B.W.X. \& Crumpton C.F. (1958). The chemical nature of silica in plants. Plant Physiology 33: 339-343.

LANNing F.C., Hopkins T.L. \& Loera J.C. (1980). Silica and ash content and depositional patterns in tissues of mature Zea mays L. plants. Annals of Botany 45: 549-554.

https://doi.org/10.1093/oxfordjournals.aob.a085859

LANSZKi J. \& MolnáR T. (2003). Diet of otters living in three different habitats in Hungary. Folia Zoologica 52: 378-388.

LANSZKi J., Heltai M. \& SzABó L. (2006). Feeding habtis and trophic niche overlap between sympatric golden jackal (Canis aureus) and red fox (Vulpes vulpes) in the Pannonian ecoregion (Hungary). Canadian Journal of Zoology 84: 1647-1656. https://doi.org/10.1139/z06-147

LANSZKi J., SÁRdi B. \& SzÉLeS G.L. (2009a). Feeding habits of the stone marten (Martes foina) in villages and farms in Hungary. Natura Somogyiensis 15: 231-246.

Lanszki J., Széles G. \& Yoxon G. (2009b). Diet composition of otters (Lutra lutra L.) living on small watercourses in southwestern Hungary. Acta Zoologica Academiae Scientiarum Hungaricae 55: 293-306.

Lanszki J., Márkus M., Újváry D., Szabó Á. \& Szemethy L. (2012). Diet of wolves Canis lupus returning to Hungary. Acta Theriologica 57: 189-193. https://doi.org/10.1007/s13364-011-0063-8

LAURIE A. \& SEIDENSTICKER J. (1977). Behavioural ecology of the sloth bear (Melursus ursinus). Journal of Zoology 182: 187-204. https://doi.org/10.1111/j.1469-7998.1977.tb04155.x

Laurimaa L., Süld K., Davison J., Moks E., Valdmann H. \& SaArma U. (2016). Alien species and their zoonotic parasites in native and introduced ranges: the raccoon dog example. Veterinary Parasitology 219: 24-33. https://doi.org/10.1016/j.vetpar.2016.01.020

LE Roux P.L. (1930). Helminthiasis of domestic stock in the Union of South Africa. Journal of South African Veterinary Medical Association 1: 43-65. 
Lee O., Lee S., Nam D.H. \& Lee H.Y. (2013). Molecular analysis for investigating dietary habits: genetic screening of prey items in scat and stomach contents of leopard cats Prionailurus bengalensis euptilurus. Zoological Studies 52: 45. https://doi.org/10.1186/1810-522X-52-45

Lee O., Lee S., Nam D.H. \& Lee H.Y. (2014). Food habits of the leopard cat (Prionailurus bengalensis euptilurus) in Korea. Mammal Study 39: 43-47. https://doi.org/10.3106/041.039.0107

Lee S.S., TSAI F.Y. \& Chen I.S. (1995). Chemical constituents from Berchemia formosana. Journal of the Chinese Chemical Society 42: 101-105. https://doi.org/10.1002/jccs.199500018

Leite M. \& Williams R. (2004). Short-eared dog Atelocynus microtis. In: Sillero-Zubiri C., Hoffman M. \& Macdonald D.W. (eds) Canids: Foxes, Wolves, Jackals, and Dogs - Species Status and Conservation Action Plan: 26-31. IUCN/SSC Canids Specialist Group, Cambridge, United Kingdom.

LEVER R.J.A. (1959). The diet of the fox since myxomatosis. Journal of Animal Ecology 28: 359-375. https://doi.org/10.2307/2089

Lima E. De S., Jorge R.S.P. \& Dalponte J.C. (2009). Habitat use and diet of bush dogs, Speothos venaticus, in the northern Pantanal, Mato Grosso, Brazil. Mammalia 73: 13-19.

https://doi.org/10.1515/MAMM.2009.002

LindSAY I.M. \& MACDONALD D.W. (1986). Behaviour and ecology of the Ruppell's fox, Vulpes ruppelli, in Oman. Mammalia 50: 461-474. https://doi.org/10.1515/mamm.1986.50.4.461

Linnaeus C. (1758). Systema Naturae, Vol. 1, Ed. 10. Impensis Direct, Laurentii Savlii, Holmiae. https://doi.org/10.5962/bhl.title.542

LiU M., S.K. PANDA \& W. LuYTEN (2020). Plant-based natural products for the discovery and development of novel anthelmintics against nematodes. Biomolecules 10: 426.

LockHART G.J. (1997). Herbal Scraps. Edited and published online by Arthur Lee Jacobson. Available from https://www.arthurleej.com/HerbalScraps.pdf [accessed 5 May 2020].

LOCKIE J.D. (1961). The food of the pine marten Martes martes in West Ross-Shire, Scotland. Proceedings of the Zoological Society of London 126: 187-195. https://doi.org/10.1111/j.1469-7998.1961.tb06171.x

Lonsdale T. (2001). Raw Meaty Bones. Revitco P/L, Windsor, Australia.

Lorica M.R.P. \& Heaney L.R. (2013). Survival of a native mammalian carnivore, the leopard cat Prionailurus bengalensis Kerr, 1792 (Carnivora: Felidae), in an agricultural landscape on an oceanic Philippine island. Journal of Threatened Taxa 5: 4451-4460.

https://doi.org/10.11609/JoTT.03352.4451-60

Loveridge A.J. \& MACDOnALD D.W. (2003). Niche separation in sympatric jackals (Canis mesomelas and Canis adustus). Journal of Zoology 259: 143-153. https://doi.org/10.1017/S0952836902003114

Lowry M.S., Stewart B.S., Heath C.B., Yochem P.K. \& Francis M. (1991). Seasonal and annual variability in the diet of California sea lions, Zalophus californianus, at San Nicolas Island, California, 1981-86. Fishery Bulletin 89: 331-336.

LuKasiK V.M. \& Alexander S.M. (2011). Spatial and temporal variation of coyote (Canis latrans) diet in Calgary, Alberta. Cities and the Environment 4: 8.

Lunney D., Triggs B., Eby P. \& Ashby E. (1990). Analysis of scats of dogs Canis familiaris and foxes Vulpes vulpes (Canidae: Carnivora) in coastal forests near Bega, New South Wales. Australian Wildlife Research 17: 61-68. https://doi.org/10.1071/WR9900061 
Lyngdoh S. \& Habib B. (2019). Predation by Himalayan wolves: Understanding conflict, culture and co-existence amongst Indo-Tibetan community and large carnivores in High Himalaya. bioRxiv preprint. Available from https://www.biorxiv.org/content/10.1101/2019.12.16.877936v1 [accessed 5 May 2020].

MacCracken J.G. \& Hansen R.M. (1982). Seasonal foods of coyotes in southeastern Idaho: A multivariate analysis. Great Basin Naturalist 42: 45-49.

MacDonald J.T. \& Nel J.A.J. (1986). Comparative diets of sympatric small carnivores. South African Journal of Wildlife Research 16: 115-121.

MAEHR D.S. \& BRADY J.R. (1986). Food habits of bobcats in Florida. Journal of Mammalogy 67: 133138. https://doi.org/10.2307/1381009

Mahmood T., Hussain I. \& Nadeem M.S. (2011). Population estimates, habitat preference and the diet of small Indian mongoose (Herpestes javanicus) in Potohar Plateau, Pakistan. Pakistan Journal of Zoology 43: 103-111.

Mallon D., Harris R.B. \& Wegge P. (2016). Snow Leopard Prey and Diet. In: McCarthy T. \& MaLlon D. (eds) Snow Leopards: 43-55. Elsevier, New York.

MANNing D.L. (2007). A comparative ecological study between coyotes (Canis latrans) in a protected and urban habitat: A closer look at enteric parasites and diet between Florida coyotes. Master Thesis. University of South Florida, Tampa, Florida.

Markov G. \& LANSZKi J. (2012). Diet composition of the golden jackal, Canis aureus in an agricultural environment. Folia Zoologica 61: 44-48. https://doi.org/10.25225/fozo.v61.i1.a7.2012

Maroyi A. (2017). Euclea undulata Thunb.: Review of its botany, ethnomedicinal uses, phytochemistry and biological activities. Asian Pacific Journal of Tropical Medicine 10: 1030-1036.

https://doi.org/10.1016/j.apjtm.2017.10.005

MaRshall W.H. (1946). Winter food habits of the pine marten in Montana. Journal of Mammalogy 27: 83-84. https://doi.org/10.2307/1375146

Matsebula S.N., Monadjem A., Roques K.G. \& Garcelon D.K. (2009). The diet of the aardwolf, Proteles cristatus at Malolotja Nature Reserve, western Swaziland. African Journal of Ecology 47: 448-451.

Matsuo R. \& OchiaI K. (2009). Dietary overlap among two introduced and one native sympatric carnivore species, the raccoon, the masked palm civet, and the raccoon dog, in Chiba Prefecture, Japan. Mammal Study 34: 187-194. https://doi.org/10.3106/041.034.0402

Mattson D.J., Podruzny S.R. \& Haroldson M.A. (2002). Consumption of fungal sporocarps by Yellowstone grizzly bears. Ursus 13: 95-103.

Mayor-Victoria R. \& Botero-Botero A. (2010). Dieta de la nutria neotropical Lontra longicaudis (Carnívora, Mustelidae) en el Río Roble, Alto Cauca, Colombia. Acta Biológica Colombiana 15: 237244.

McGrosky A., Navarrete A., Isler K., Langer P. \& Clauss M. (2016). Gross intestinal morphometry and allometry in Carnivora. European Journal of Wildlife Research 62: 395-405.

https://doi.org/10.1007/s10344-016-1011-3

McKenzie S.J., Brown W.Y. \& Price I.R. (2010). Reduction in grass eating behaviours in the domestic dog, Canis familiaris, in response to a mild gastrointestinal disturbance. Applied Animal Behaviour Science 123: 51-55. https://doi.org/10.1016/j.applanim.2009.12.003

MClanahan H.M. (1918). Intestinal parasites in children. Journal of the American Medical Association 71: 623-625. 
MCLellan B.N. (2011). Implications of a high-energy and low-protein diet on the body composition, fitness, and competitive abilities of black (Ursus americanus) and grizzly (Ursus arctos) bears. Canadian Journal of Zoology 89: 546-558. https://doi.org/10.1139/z11-026

McLennan M.R. \& Huffman M.A. (2012). High frequency of leaf swallowing and its relationship to intestinal parasite expulsion in "village" chimpanzees at Bulindi, Uganda. American Journal of Primatology 74: 642-650. https://doi.org/10.1002/ajp.22017

McShea W.J., Sheng L., Xiaoli S., Fang W. \& Dajun W. (2018). Guide to the Wildlife of Southwest China. Smithsonian Institution Scholarly Press, Washington, D.C.

Mech L.D. (1966). The wolves of Isle Royale. Fauna of the National Parks of the United States Fauna Series 7: 1-210.

Mehra P.N. \& Sharma O.P. (1965). Epidermal silica cells in the Cyperaceae. Botanical Gazette 126: 53-58.

Melville H.I.A.S., Bothma J. Du P. \& Mills M.G.L. (2004). Prey selection by caracal in the Kgalagadi Transfrontier Park. South African Journal of Wildlife Research 34: 67-75.

Mesa-Cruz J.B., Brown J.L., Waits L.P. \& Kelly M.J. (2016). Non-invasive genetic sampling reveals diet shifts, but little difference in endoparasite richness and faecal glucocorticoids, in Belizean felids inside and outside protected areas. Journal of Tropical Ecology 32: 226-239.

https://doi.org/10.1017/S0266467416000213

Mills M.G.L. \& Mills M.E.J. (1978). The diet of the brown Hyaena Hyaena brunnea in the southern Kalaharia. Koedoe 21: 125-149. https://doi.org/10.4102/koedoe.v21i1.968

Mohamed A., Samejima H. \& Wilting A. (2009). Records of five Bornean cat species from Deramakot Forest Reserve in Sabah, Malaysia. CATnews 51: 12-15.

Molsher R., Newsome A. \& Dickman C. (1999). Feeding ecology and population dynamics of the feral cat (Felis catus) in relation to the availability of prey in central-eastern New South Wales. Wildlife Research 26: 593-607. https://doi.org/10.1071/WR98058

Mondal P.C.K., SANKar K. \& Qureshi Q. (2012). Food habits of golden jackal (Canis aureus) and striped hyena (Hyaena hyaena) in Sariska Tiger Reserver, western India. World Journal of Zoology 7 : $106-112$.

Monroy-Vilchis O., Gómez Y., Janczur M. \& Urios V. (2009). Food niche of Puma concolor in central Mexico. Wildlife Biology 15: 97-105. https://doi.org/10.2981/07-054

Montalvo V., Sáenz-Bolaños C., Cruz J.C., Hagnauer I. \& Carrillo E. (2020). Consumption of wild rice (Oryza latifolia) by free-ranging jaguars, pumas, and ocelots (Carnivora-Felidae) in northwestern Costa Rica. Food Webs 22: e00138.

Morell T. (1774). An Abridgement of the Last Quarto Edition of Ainsworth's Dictionary, English and Latin. Charles Rivington, London.

Moreno R.S., KaYs R.W. \& Samudio Jr. R. (2006). Competitive release in diets of ocelot (Leopardus pardalis) and puma (Puma concolor) after jaguar (Panthera onca) decline. Journal of Mammology 87: 808-816. https://doi.org/10.1644/05-MAMM-A-360R2.1

Morey P.S., GeSE E.M. \& GeHRT S. (2007). Spatial and temporal variation in the diet of coyotes in the Chicago metropolitan area. The American Midland Naturalist 158: 147-161.

https://doi.org/10.1674/0003-0031(2007)158[147:SATVIT]2.0.CO;2 
Motta-Junior J.C., Talamoni S.A., Lombardi J.A. \& Simokomaki K. (1996). Diet of the maned wolf, Chrysocyon brachyurus, in central Brazil. Journal of Zoology 240: 277-284.

https://doi.org/10.1111/j.1469-7998.1996.tb05284.x

Mudappa D., Kumar A. \& Chellam R. (2010). Diet and fruit choice of the brown palm civet Paradoxurus jerdoni, a viverrid endemic to the Western Ghats rainforest, India. Tropical Conservation Science 3: 282-300. https://doi.org/10.1177/194008291000300304

MüLler S. (2006). Diet Composition of Wolves (Canis lupus) on the Scandinavian Peninsula determined by Scat Analysis. Diploma Thesis. Technical University of Munich, Munich, Germany.

MüLLER S.T.M. (2016). Hábitos alimentares e conservação do lobo-guará (Chrysocyon brachyurus) (Illiger, 1815) em um remanescente de Cerrado em Pirassununga - SP. Doctoral dissertation. Universidade Federal de Sāo Carlos, Sāo Carlos, Brazil.

Mullu D. \& Balakrishnan M. (2014). Ecology of African civet (Civettictis civetta) in Arba Minch Forest, Arba Minch, Ethiopia. Science, Technology and Arts Research Journal 3: 99-102.

Murie A. (1944). The Wolves of Mount McKinley. Fauna of the National Parks of the United States, Fauna Series, 5. Government Printing Office, Washington, D.C.

MurIE O.J. (1935). Food Habits of the Coyote in Jackson Hole, Wyo. Circular No. 362. United States Department of Agriculture, Washington D.C.

MuRIE O.J. (1945). Notes on coyote food habits in Montana and British Columbia. Journal of Mammology 26: 33-40. https://doi.org/10.2307/1375030

Mustonen A.M., Lempiäinen T., Aspelund M., Hellstedt P., Ikonen K., ItÄmies J., VÄhÄ V., Erkinaro J., Asikainen J., Kunnasranta M. \& Niemelä P. (2012). Application of change-point analysis to determine winter sleep patterns of the raccoon dog (Nyctereutes procyonoides) from body temperature recordings and a multi-faceted dietary and behavioral study of wintering. BMC Ecology 12: 27.

https://doi.org/10.1186/1472-6785-12-27

Myhre R. \& Myrberget S. (1975). Diet of wolverines (Gulo gulo) in Norway. Journal of Mammalogy 56: 752-757. https://doi.org/10.2307/1379650

Nadeem M.S., Naz R., Shah S.I., Beg M.A., Kayani A.R., Mushtaq M. \& Mahmood T. (2012). Season- and locality-related changes in the diet of Asiatic jackal (Canis aureus) in Potohar, Pakistan. Turkish Journal of Zoology 36: 798-805.

NAKWAYA D.N. (2009). The Diet of the Black Mongoose (Galerella nigrata) (Carnivora: Herpestidae) in North-west (Hobatere Concession) and North-central (Erongo Conservancy), Namibia. Master Thesis. University of Namibia, Windhoek, Namibia.

Napoli E., Anile S., Arrabito C., Scornavacca D., Mazzamuto M. V., Gaglio G., Otranto D., GiannetTo S. \& BRianti E. (2016). Survey on parasitic infections in wildcat (Felis silvestris silvestris Schreber, 1777) by scat collection. Parasitology Research 115: 255-261.

https://doi.org/10.1007/s00436-015-4742-2

Nava-Vargas V., Tejero J.D. \& Chávez C.B. (1999). Hábitos alimentarios del cacomixtle Bassariscus astutus (Carnivora: Procyonidae) en un matorral xerófilo de Hidalgo, México. Anales del Instituto de Biología Universidad Nacional Autónoma de México 70: 51-63.

Ndjonka D., Abladam E.D., Djafsia B., Ajonina-Ekoti I., Achukwi M.D. \& Liebau E. (2014). Anthelmintic activity of phenolic acids from the axlewood tree Anogeissus leiocarpus on the filarial nematode Onchocerca ochengi and drug-resistant strains of the free-living nematode Caenorhabditis elegans. Journal of Helminthology 88: 481-488. https://doi.org/10.1017/S0022149X1300045X 
Nilssen K.T., Haug T., ØRitsland T., Lindblom L. \& Kjellqwist S.A. (1998). Invasions of harp seals Phoca groenlandica Erxleben to coastal waters of Norway in 1995: Ecological and demographic implications. Sarsia 83: 337-345. https://doi.org/10.1080/00364827.1998.10413693

Nilssen K.T., Haug T. \& Lindblom C. (2001). Diet of weaned pups and seasonal variations in body condition of juvenile Barents Sea harp seals Phoca groenlandica. Marine Mammal Science 17: 926936. https://doi.org/10.1111/j.1748-7692.2001.tb01306.x

Nishida A.H. \& Ochman H. (2018). Rates of gut microbiome divergence in mammals. Molecular Ecology 27: 1884-1897. https://doi.org/10.1111/mec.14473

NowaK R.M. (2005). Walker's Carnivores of the World ( $6^{\text {th }}$ ed.). The Johns Hopkins University Press, Baltimore, Maryland.

Nowak S., Mysıajek R.W., Kıosińska A. \& Gabryś G. (2011). Diet and prey selection of wolves (Canis lupus) recolonising Western and Central Poland. Mammalian Biology 76: 709-715. https://doi.org/10.1016/j.mambio.2011.06.007

Nussbaum R.A. \& Maser C. (1975). Food habits of the bobcat, Lynx rufus, in the Coast and Cascade ranges of western Oregon in relation to present management policies. Northwest Science 49: 261-266.

Nyakatura K. \& Bininda-Emonds O.R. (2012). Updating the evolutionary history of Carnivora (Mammalia): a new species-level supertree complete with divergence time estimates. BMC Biology 10: 12. https://doi.org/10.1186/1741-7007-10-12

Nybakken L., Helmersen A.M., Gauslaa Y. \& Selås V. (2010). Lichen compounds restrain lichen feeding by bank voles (Myodes glareolus). Journal of Chemical Ecology 36: 298-304.

https://doi.org/10.1007/s10886-010-9761-y

OKawara Y., NaKanishi N. \& Izawa M. (2020). Short-term fruiting phenology affects the diet of an omnivorous carnivoran, the Tsushima marten Martes melampus tsuensis. Mammal Study 45: 49-61. https://doi.org/10.3106/ms2019-0032

Oli M.K., TAYlor I.R. \& Rogers M.E. (1993). Diet of the snow leopard (Panthera uncia) in the Annapurna Conservation Area, Nepal. Journal of Zoology 231: 365-370.

https://doi.org/10.1111/j.1469-7998.1993.tb01924.x

Ortiz-Alcaraz A., Arnaud G., Aguirre-Muñoz A., Galina-Tessaro P., MÉndez-Sánchez F. \& Ortega-Rubio A. (2017). Diet and home-range of the feral cat, Felis catus (Carnivora: Felidae) on Socorro Island, Revillagigedo Archipelago, Mexico. Acta Zoológica Mexicana 33: 482-489.

Ott T., Kerley G.I. \& Boshoff A.F. (2007). Preliminary observations on the diet of leopards (Panthera pardus) from a conservation area and adjacent rangelands in the Baviaanskloof region, South Africa. African Zoology 42: 31-37. https://doi.org/10.1080/15627020.2007.11407374

Pablo-Rodríguez N., Aurioles-Gamboa D. \& Montero-Muñoz J.L. (2016). Niche overlap and habitat use at distinct temporal scales among the California sea lions (Zalophus californianus) and Guadalupe fur seals (Arctocephalus philippii townsendi). Marine Mammal Science 32: 466-489. https://doi.org/10.1111/mms.12274

Padial J.M., Ávila E. \& Gil-Sánchez J.M. (2002). Feeding habits and overlap among red fox (Vulpes vulpes) and stone marten (Martes foina) in two Mediterranean mountain habitats. Mammalian Biology 67: 137-146. https://doi.org/10.1078/1616-5047-00021

Pagh S. \& Hersteinsson P. (2008). Difference in diet and age structure of blue and white Arctic foxes (Vulpes lagopus) in the Disko Bay area, West Greenland. Polar Research 27: 44-51.

https://doi.org/10.1111/j.1751-8369.2008.00042.x 
Palmer R. (2012). Diet of the dingo (Canis lupus dingo) from the desert uplands of North Queensland. Queensland Naturalist 50: 23-26.

Palmer R. \& Fairall N. (1988). Caracal and African wild cat diet in the Karoo National Park and the implications thereof for hyrax. South African Journal of Wildlife Research 18: 30-34.

Panthi S., Aryal A., Raubenheimer D., Lord J. \& Adhikari B. (2012). Summer diet and distribution of the red panda (Ailurus fulgens fulgens) in Dhorpatan Hunting Reserve, Nepal. Zoological Studies 51: 701-709.

Papageorgiou N., Vlachos C., Sfougaris A. \& Tsachalidis E. (1994). Status and diet of wolves in Greece. Acta Theriologica 39: 411-416.

PAPeş M. \& GAUBert P. (2007). Modelling ecological niches from low numbers of occurrences: assessment of the conservation status of poorly known viverrids (Mammalia, Carnivora) across two continents. Diversity and Distributions 13: 890-902. https://doi.org/10.1111/j.1472-4642.2007.00392.x

PARR J.W.K. \& DucKWorth J.W. (2007). Notes on diet, habituation and sociality of yellow-throated marten Martes flavigula. Small Carnivore Conservation 36: 27-29.

Parsons A.W., Simons T.R., O’Connell A.F. \& Stoskopf M.K. (2013). Demographics, diet, movements, and survival of an isolated, unmanaged raccoon Procyon lotor (Procyonidae, Carnivora) population on the Outer Banks of North Carolina. Mammalia 77: 21-30.

https://doi.org/10.1515/mammalia-2011-0138

PATERSON JR. R.L. (1984). High incidence of plant material and small mammals in the autumn diet of turkey vultures in Virginia. The Wilson Bulletin 96: 467-469.

PAulini J. (1834). Dissertatio Inauguralis Physiologico-Pathologica de Autocratia. Tipis Landererianis, Pestini.

Pedó E., Tomazzoni A.C., Hartz S.M. \& Christoff A.U. (2006). Diet of crab-eating fox, Cerdocyon thous (Linnaeus) (Carnivora, Canidae), in a suburban area of southern Brazil. Revista Brasileira de Zoologia 23: 637-641. https://doi.org/10.1590/S0101-81752006000300005

Peters K., Gorzolka K., Bruelheide H. \& Neumann S. (2018). Seasonal variation of secondary metabolites in nine different bryophytes. Ecology and Evolution 8: 9105-9117.

https://doi.org/10.1002/ece3.4361

Peterson R. O. \& Ciucci P. (2003). The wolf as a carnivore. In: Mech L.D. \& Boitani L. (eds) Wolves: Behavior, Ecology, and Conservation: 104-130. The University of Chicago Press, Chicago, Illinois.

Phillips R.B., Winchell C.S. \& Schmidt R.H. (2007). Dietary overlap of an alien and native carnivore of San Clemente Island, California. Journal of Mammalogy 88: 173-180.

https://doi.org/10.1644/06-MAMM-A-015R2.1

Pineda-Munoz S. \& Alroy J. (2014). Dietary characterization of terrestrial mammals. Proceedings of the Royal Society B: Biological Sciences 281: 20141173. https://doi.org/10.1098/rspb.2014.1173

Pires M.M., Widmer C.E., Silva C. \& Setz E.Z.F. (2011). Differential detectability of rodents and birds in scats of ocelots, Leopardus pardalis (Mammalia: Felidae). Zoologia 28: 280-283.

https://doi.org/10.1590/S1984-46702011000200019

Powell A.N.W. (1957). Call of the Tiger. Robert Hale Limited, London.

Prins H.H.T. (1982). Why are mosses eaten in cold environments only? Oikos 38: 374-380. https://doi.org/10.2307/3544680 
Prugh L.R., Arthur S.M. \& Ritland C.E. (2008). Use of faecal genotyping to determine individual diet. Wildlife Biology 14: 318-330.

https://doi.org/10.2981/0909-6396(2008)14[318:UOFGTD]2.0.CO;2

Pulliainen E. \& OllinmäKi P. (1996). A long-term study of the winter food niche of the pine marten Martes martes in northern boreal Finland. Acta Theriologica 41: 337-352.

Quadros J. \& MonteIro-Filho E.L. (2000). Fruit occurrence in the diet of the Neotropical otter, Lontra longicaudis in southern Brazilian Atlantic forest and its implication for seed dispersion. Mastozoología Neotropical 7: 33-36.

QueIrolo D. \& MotTA-Junior J.C. (2007). Prey availability and diet of maned wolf in Serra da Canastra National Park, southeastern Brazil. Acta Theriologica 52: 391-402.

https://doi.org/10.1007/BF03194237

Quick H.F. (1955). Food habits of marten (Martes americana) in northern British Columbia. The Canadian Field-Naturalist 69: 144-147.

Quinlan M.B., QuinLan R.J. \& Nolan J.M. (2002). Ethnophysiology and herbal treatments of intestinal worms in Dominica, West Indies. Journal of Ethnopharmacology 80: 75-83.

https://doi.org/10.1016/S0378-8741(02)00002-8

Quintela F.M., Artioli L.G.S. \& Porciuncula R.A. (2012). Diet of Lontra longicaudis (Olfers, 1818) (Carnivora: Mustelidae) in three limnic systems in southern Rio Grande do Sul state, Brazil. Brazilian Archives of Biology and Technology 55: 877-886. https://doi.org/10.1590/S1516-89132012000600011

Rajaratnam R., Sunquist M., Rajaratnam L. \& Ambu L. (2007). Diet and habitat selection of the leopard cat (Prionailurus bengalensis borneoensis) in an agricultural landscape in Sabah, Malaysian Borneo. Journal of Tropical Ecology 23: 209-217. https://doi.org/10.1017/S0266467406003841

RAMESh T., SANKAR K. \& QuReShi Q. (2009). Additional notes on the diet of sloth bear Melursus ursinus in Mudumalai Tiger Reserve as shown by scat analysis. Journal of the Bombay Natural Historical Society 106: 204-206.

RigG R. \& Gorman M. (2004). Spring-autumn diet of wolves (Canis lupus) in Slovakia and a review of wolf prey selection. Oecologia Montana 13: 30-41.

Roberts P.D., Somers M.J., White R.M. \& Nel J.A.J. (2007). Diet of the South African large-spotted genet Genetta tigrina (Carnivora, Viverridae) in a coastal dune forest. Acta Theriologica 52: 45-53. https://doi.org/10.1007/BF03194198

RobinetTe W.L., Gashwiler J.S. \& Morris O.W. (1959). Food habits of the cougar in Utah and Nevada. The Journal of Wildlife Management 23: 261-273.

Rocha-Mendes F., Mikich S.B., Quadros J. \& Pedro W.A. (2010). Feeding ecology of carnivores (Mammalia, Carnivora) in Atlantic Forest remnants, southern Brazil. Biota Neotropica 10: 21-30. https://doi.org/10.1590/S1676-06032010000400001

Roldos V., Nakayama H., Rolón M., Montero-Torres A., Trucco F., Torres S., Vega C., MarreroPonce Y., Heguaburu V., Yaluff G., Gómez-Barrio A., Sanabria L., Ferreira M.E., de Arias A.R. \& PANDOLFI E. (2008). Activity of a hydroxybibenzyl bryophyte constituent against Leishmania spp. and Trypanosoma cruzi: In silico, in vitro and in vivo activity studies. European Journal of Medicinal Chemistry 43: 1797-1807. https://doi.org/10.1016/j.ejmech.2007.11.007

Rollings C.T. (1945). Habits, foods and parasites of the bobcat in Minnesota. The Journal of Wildlife Management 9: 131-145. https://doi.org/10.2307/3795892 
RomANowski J. \& LesiŃski G. (1991). A note on the diet of stone marten in southeastern Romania. Acta Theriologica 36: 201-204.

Romero-Benavides J.C., Ruano A.L., Silva-Rivas R., Castillo-Veintimilla P., VivancoJARAMILlo S., \& BaILON-Moscoso N. (2017). Medicinal plants used as anthelmintics: Ethnomedical, pharmacological, and phytochemical studies. European Journal of Medicinal Chemistry 129: 209-217. https://doi.org/10.1016/j.ejmech.2017.02.005

Romo M.C. (1995). Food habits of the Andean fox (Pseudalopex culpaeus) and notes on the moutain cat (Felis colocolo) and puma (Felis concolor) in the Rio Abiseo National Park, Peru. Mammalia 59: 335-344. https://doi.org/10.1515/mamm.1995.59.3.335

Roper T.J. \& Mickevicius E. (1995). Badger Meles meles diet: a review of literature from the former Soviet Union. Mammal Review 25: 117-129. https://doi.org/10.1111/j.1365-2907.1995.tb00451.x

Rosalino L.M. \& Santos-Reis M. (2009). Fruit consumption by carnivores in Mediterranean Europe. Mammal Review 39: 67-78. https://doi.org/10.1111/j.1365-2907.2008.00134.x

Ross G.J., Shaughnessy P.D. \& Best P.B. (1978). New records of crabeater seals (Lobodon carcinophagus) from South Africa. Annals of the South African Museum 75: 153-158.

Ross S. (2009). Providing an Ecological Basis for the Conservation of the Palla's Cat (Otocolobus manul). Ph.D. dissertation. University of Bristol, Bristol, United Kingdom.

Rowe-Rowe D.T. (1983). Black-backed jackal diet in relation to food availability in the Natal Drakensberg. South African Journal of Wildlife Research 13: 17-23.

Russell R.H. (1975). The food habits of polar bears of James Bay and southwest Hudson Bay in summer and autumn. Arctic 28: 117-129.

SÁnchez E., Montiel M. \& EsPinoza A.M. (2003). Ultrastructural morphologic description of the wild rice species Oryza latifolia (Poaceae) in Costa Rica. Revista de Biología Tropical 51: 345-354.

SÁnchez M., Rodrigues P., Ortuño V. \& Herrero J. (2008). Feeding habits of the genet Genetta genetta in an Iberian continental wetland. Hystrix, the Italian Journal of Mammalogy 19: 133-142.

Sankar K. \& Johnsingh A.J.T. (2002). Food habits of tiger (Panthera tigris) and leopard (Panthera pardus) in Sariska Tiger Reserve, Rajasthan, India, as shown by scat analysis. Mammalia 66: 285-288. https://doi.org/10.1515/mamm.2002.66.2.285

Santana E.M. (2010). Food Habits and Anthropogenic Supplementation in the Diet of Coyotes (Canis latrans) along an Urban-Rural Gradient. M.S. Thesis. Auburn University, Auburn, Alabama.

SAntana E.M. \& Armstrong J.B. (2017). Food habits and anthropogenic supplementation in coyote diets along an urban-rural gradient. Human-Wildlife Interactions 11: 156-166.

Santos E.F., Setz E.Z. \& Gobbi N. (2003). Diet of the maned wolf (Chrysocyon brachyurus) and its role in seed dispersal on a cattle ranch in Brazil. Journal of Zoology 260: 203-208.

https://doi.org/10.1017/S0952836903003650

Sarmento P. (1996) Feeding ecology of the European wildcat Felis silvestris in Portugal. Acta Theriologica 41: 409-414.

Schaller G., Tserendeleg J. \& AmarsanaA G. (1994). Observations on snow leopards in Mongolia. Proceedings of the International Snow Leopard Symposium 7: 33-42.

Schaller G.B. (1967). The Deer and the Tiger. The University of Chicago Press, Chicago, Illinois. 
Schaller G.B., Qitao T., Johnson K.G., Xiaoming W., Heming S. \& Jinchu H. (1989). The feeding ecology of giant pandas and Asiatic black bears in the Tangjiahe Reserve, China. In: GitTleman J.L. (ed.) Carnivore Behavior, Ecology, and Evolution: 212-241. Springer, Boston, Massachusetts. https://doi.org/10.1007/978-1-4613-0855-3_9

SCHEFFER T.H. \& SPERry C.C. (1931). Food habits of the Pacific harbor seal, Phoca richardii. Journal of Mammalogy 12: 214-226. https://doi.org/10.2307/1373868

SCHEMPP H., Weiser D. \& ElSTNER E.F. (2000). Biochemical model reactions indicative of inflammatory processes. Arzneimittelforschung 50: 362-372. https://doi.org/10.1055/s-0031-1300215

Schoonover L.J. \& Marshall W.H. (1951). Food habits of the raccoon (Procyon lotor hirtus) in northcentral Minnesota. Journal of Mammalogy 32: 422-428. https://doi.org/10.2307/1375790

Scott M.D. (1971). Ecology and Ethology of Feral Dogs in East-central Alabama. Ph.D. dissertation. Auburn University, Auburn, Alabama.

SCOtT M.D. \& CAuSEY K. (1973). Ecology of feral dogs in Alabama. Journal of Wildlife Management 37: 253-265. https://doi.org/10.2307/3800116

Scott T.G. (1942). An Investigation of the Food Coactions of the Northern Plains Red Fox. Ph.D. dissertation. Iowa State College, Ames, Iowa.

Selko L.F. (1937). Food habits of Iowa skunks in the fall of 1936. The Journal of Wildlife Management 1: 70-76. https://doi.org/10.2307/3795763

Seryodkin I.V. \& Burkovskiy O.A. (2019). Food habit analysis of the amur leopard cat Prionailurus bengalensis euptilurus in the Russian Far East. Biology Bulletin 46: 648-653.

Sethy J. \& Chauhan N.P.S. (2018). Dietary preference of Malayan sun bear Helarctos malayanus in Namdapha Tiger Reserver, Arunachal Pradesh, India. Wildlife Biology 2018: wlb.00351. https://doi.org/10.2981/wlb.00351

Sharma H.P., Swenson J.E. \& Belant J.L. (2014). Seasonal food habits of the red panda (Ailurus fulgens) in Rara National Park, Nepal. Hystrix 25: 47-50.

Short H.L. (1979). Food Habits of Coyotes in a Semidesert Grass-shrub Habitat. Rocky Mountain Forest and Range Experiment Station, Forest Service, U.S. Department of Agriculture, Research Note RM 364.

Sidorovich V.E., Krasko D.A. \& Dyman A.A. (2005). Landscape-related differences in diet, food supply and distribution pattern of the pine marten, Martes martes in the transitional mixed forest of northern Belarus. Folia Zoologica 54: 39-52.

Silva-Pereira J.E., Moro-Rios R.F., Bilski D.R. \& Passos F.C. (2011). Diets of three sympatric Neotropical small cats: Food niche overlap and interspecies differences in prey consumption. Mammalian Biology 76: 308-312. https://doi.org/10.1016/j.mambio.2010.09.001

Simpson F. (1902). Cats and All About Them. Frederick A. Stokes Company, New York.

Sims J.A., Parsons J.L., Bissell H.A., Sikes R.S., Ouellette J. R. \& Rude B.J. (2007). Determination of bamboo-diet digestibility and fecal output by giant pandas. Ursus 18: 38-46.

https://doi.org/10.2192/1537-6176(2007)18[38:DOBDAF]2.0.CO;2

Sin T., Gazzola A., Chiriac S., \& Rîsnoveanu G. (2019). Wolf diet and prey selection in the SouthEastern Carpathian Mountains, Romania. PLOS ONE: e0225424.

https://doi.org/10.1371/journal.pone.0225424 
Śmietana W. \& KlimeK A. (1993). Diet of wolves in the Bieszczady Mountains, Poland. Acta Theriologica 38: 245-251.

Smythe N. (1986). Competition and resource partitioning in the guild of neotropical terrestrial frugivorous mammals. Annual Review of Ecology and Systematics 17: 169-188.

SNEAd E. \& HeNDRICKSON G.O. (1942). Food habits of the badger in Iowa. Journal of Mammalogy 23: 380-391. https://doi.org/10.2307/1375048

Souther O. \& Wiggers S. (2012). Comparative diet analysis of Canis rufus and Canis latrans. Proceedings of the National Conference on Undergraduate Research (Weber State University, Ogden, Utah): 313-317.

Sovada M.A., Roaldson J.M. \& Sargeant A.B. (1999). Foods of American badgers in west-central Minnesota and southeastern North Dakota during the duck nesting season. The American Midland Naturalist 142: 410-414.

Sovada M.A., Roy C.C. \& Telesco D.J. (2001). Seasonal food habits of swift fox (Vulpes velox) in cropland and rangeland landscapes in western Kansas. American Midland Naturalist 145: 101-111.

Spiegler V., Liebau E. \& Hensel A. (2017). Medicinal plant extracts and plant-derived polyphenols with anthelmintic activity against intestinal nematodes. Natural Product Reports 34: 627.

https://doi.org/10.1039/c6np00126b

ŠPinkytė-BaČKaitiené R. \& PĖtelis K. (2012). Diet composition of wolves (Canis lupus L.) in Lithuania. Acta Biologica Universitatis Daugavpiliensis 12: 100-105.

Stahler D.R., Smith D.W. \& GuernSEY D.S. (2006). Foraging and feeding ecology of the gray wolf (Canis lupus): lessons from Yellowstone National Park, Wyoming, USA. The Journal of Nutrition 136: 1923S-1926S. https://doi.org/10.1093/jn/136.7.1923S

Steenhuisen S.L., Balmer A., Zoeller K., Kuhn N., Midgley J., Hansen D. \& Johnson S.D. (2015). Carnivorous mammals feed on nectar of Protea species (Proteaceae) in South Africa and likely contribute to their pollination. African Journal of Ecology 53: 602-605.

Stempniewicz L. (2017). Polar bears observed climbing steep slopes to graze on scurvy grass in Svalbard. Polar Research 36: 1326453. https://doi.org/10.1080/17518369.2017.1326453

StEnLund M.H. (1955). A Field Study of the Timber Wolf (Canis lupus) on the Superior National Forest, Minnesota. Technical Bulletin No. 4. Minnesota Department of Conservation.

StePkovitch B. (2017). Feeding Ecology of an Invasive Predator across an Urban Land Use Gradient. M.R. Thesis. Western Sydney University, Sydney, Australia.

Still G.F. (1908). Hairball in the stomach. Proceedings of the Royal Society of Medicine 1: 212-215.

Stiner M.C., Munro N.D. \& SAnZ M. (2012). Carcass damage and digested bone from mountain lions (Felis concolor): implications for carcass persistence on landscapes as a function of prey age. Journal of Archaeological Science 39: 896-907. https://doi.org/10.1016/j.jas.2011.10.020

Stoel P.F. (1976). Some Coyote Food Habit Patterns in the Shrub-steppe of South-central Washington. M.S. Thesis. Portland State University, Portland, Oregon.

Stoyanov S. (2012). Golden jackal (Canis aureus) in Bulgaria. Current status, distribution, demography and diet. Proceedings of International Symposium on Hunting 1: 48-56.

Stuart C.T. (1976a). Diet of the black backed jackal Canis mesomelas in the Central Namib Desert, south west Africa. Zoologica Africna 11: 193-205. 
StUART C.T. (1976b). Analysis of Felis libyca and Genetta genetta scats from the Central Namib Desert, south west Africa. Zoologica Africana 12: 239-241.

Stuart C.T. \& Stuart T. (1998). Notes on the diet of four species of viverrid in a limited area of southern Namaqualanad, Northern Cape, South Africa. Small Carnivore Conservation 19: 9-10.

Stuart C.T. \& Stuart T. (2003). A short note on the analysis of the scats of water mongoose Atilax paludinosus and rusty-spotted genet Genetta maculata from Kasanka National Park, north-east Zambia. Small Carnviore Conservation 29: 15.

Stuart C.T., Stuart T. \& Pereboom V. (2003). Diet of the bat-eared fox (Otocyon megalotis), based on scat analysis, on the Western Escarpment, South Afirca. Canid News 6: 2.

Su H., Su Y. \& Huffman M.A. (2013). Leaf swallowing and parasitic infection of the Chinese lesser civet Viverricula indica in northeastern Taiwan. Zoological Studies 52: 22.

https://doi.org/10.1186/1810-522X-52-22

Sueda K.L.C., Hart B.L. \& Cliff K.D. (2007). Characterisation of plant eating in dogs. Applied Animal Behaviour Science 111: 120-132. https://doi.org/10.1016/j.applanim.2007.05.018

Süld K., Valdmann H., Laurimaa L., Soe E., Davison J. \& Saarma U. (2014). An invasive vector of zoonotic disease sustatined by anthropogenic resources: the raccoon dog in northern Europe. PLOS ONE 9: e96358. https://doi.org/10.1371/journal.pone.0096358

SunQuist M.E. (1981). The social organization of tigers (Panthera tigris) in Royal Chitawan National Park, Nepal. Smithsonian Contributions to Zoology 336: 1-98.

Sutor A., Kauhala K. \& Ansorge H. (2010). Diet of the raccoon dog Nyctereutes procyonoides - a canid with an opportunistic foraging strategy. Acta Theriologica 55: 165-176.

https://doi.org/10.4098/j.at.0001-7051.035.2009

SzCZEPANIAK M. (2009). Ecological aspects of anatomical and morphological variation of Elymus hispidus, E. repens and E. xmucronatus. Grass Research: 49-67.

Taber A.B., Novaro A.J., Neris N. \& Colman F.H. (1997). The food habits of sympatric jaguar and puma in the Paraguayan Chaco. Biotropica 29: 204-213.

https://doi.org/10.1111/j.1744-7429.1997.tb00025.x

Tamimi N., Malmasi A., Talebi A., Tamimi F. \& Amini A. (2013). Owner complaints of canine behavior in Iran - A preliminary survey. Journal of Veterinary Behavior 8: 26-31.

https://doi.org/10.1016/j.jveb.2012.05.007

TATARA M. \& Dor T. (1994). Comparative analyses on food habits of Japanese marten, Siberian weasel and leopard cat in the Tsushima islands, Japan. Ecological Research 9: 99-107.

https://doi.org/10.1007/BF02347247

TAYLOR W.P. (1954). Food habits and notes on life history of the ring-tailed cat in Texas. Journal of Mammalogy 35: 55-63. https://doi.org/10.2307/1376073

Thompson D.Q. (1952). Travel, range, and food habits of timber wolves in Wisconsin. Journal of Mammalogy 33: 429-442. https://doi.org/10.2307/1376015

Tidemann C.R., Yorkston H.D. \& Russack A.J. (1994). The diet of cats, Felis catus, on Christmas Island, Indian Ocean. Wildlife Research 21: 279-286. https://doi.org/10.1071/WR9940279

Tófoli C.F., Rohe F. \& SeTz E.Z.F. (2009). Jaguarundi (Puma yagouaroundi) (Geoffroy, 1803) (Carnivora, Felidae) food habits in a mosaic of Atlantic Rainforest and eucalypt plantations of southeastern Brazil. Brazilian Journal of Biology 69: 871-877. https://doi.org/10.1590/S1519-69842009000400015 
Toweill D.E. \& Maser C. (1985). Food of cougars in the Cascade Range of Oregon. The Great Basin Naturalist 45: 77-80.

TrAPP G.R. (1978). Comparative behavioral ecology of ringtail and gray fox in southwestern Utah. Carnivore 1: 3-32.

Trembath-Reichert E., Wilson J.P., McGlynn S.E. \& Fischer W.W. (2015). Four hundred million years of silica biomineralization in land plants. Proceedings of the National Academy of Sciences 112: 5449-5454. https://doi.org/10.1073/pnas.1500289112

Troya V., Cuesta F. \& Peralvo M. (2004). Food habits of Andean bears in the Oyacachi River Basin, Ecuador. Ursus 15: 57-60. https://doi.org/10.2192/1537-6176(2004)015<0057:FHOABI>2.0.CO;2

Tsuji Y., YASUmoto Y. \& TAKATSUKi S. (2014). Multi-annual variation in the diet composition and frugivory of the Japanese marten (Martes melampus) in western Tokyo, central Japan. Acta Theriologica 59: 479-483. https://doi.org/10.1007/s13364-014-0181-1

Tucker A.O. \& Tucker S.S. (1988). Catnip and the catnip response. Economic Botany 42: 214-231. https://doi.org/10.1007/BF02858923

Tugwell S. \& Branch G.M. (1992). Effects of herbivore gut surfactants on kelp polyphenol defenses. Ecology 73: 205-215. https://doi.org/10.2307/1938732

Turkowski F.J. (1980). Carnivora Food Habits and Habitat Use in Ponderosa Pine Forests. Research Paper RM-215. Rocky Mountain Forest and Range Experiment Station, Forest Service, U.S. Department of Agriculture.

Urban A. (2016). Truffles and small mammals. In: Zambonelli A., Iotti M. \& Murat C. (eds) True Truffle (Tuber spp.) in the World: 353-373. Springer, Switzerland.

Valdmann H., Andersone-Lilley Z., Koppa O., Ozolins J. \& Bagrade G. (2005). Winter diets of wolf Canis lupus and lynx Lynx lynx in Estonia and Latvia. Acta Theriologica 50: 521-527.

https://doi.org/10.1007/BF03192645

VAN AARDE R.J. (1980). The diet and feeding behaviour of feral cats, Felis catus at Marion Island. South African Journal of Wildlife Research 10: 123-128.

van Dijk J., Hauge K., Landa A., Andersen R. \& May R. (2007). Evaluating scat analysis methods to assess wolverine Gulo gulo diet. Wildlife Biology 13 (supplement 2): 62-67.

https://doi.org/10.2981/0909-6396(2007)13[62:ESAMTA]2.0.CO;2

VILJOEN S. \& DaVIS D.H.S. (1973). Notes on stomach contents analyses of various carnivores in southern Africa (Mammalia: Carnivora). Annals of the Transvaal Museum 28: 353-363.

Viro P. \& Mikkola H. (1981). Food composition of the raccoon dog Nyctereutes procyonoides Gray, 1834 in Finland. Zeitschrift für Säugetierkunde 46: 20-26.

Vos J. (2000). Food habits and livestock depredation of two Iberian wolf packs (Canis lupus signatus) in the north of Portugal. Journal of Zoology 251: 457-462.

Vulla E., Hobson K.A., Korsten M., Leht M., Martin A.-J., Lind A., Männil P., Valdmann H. \& SAARMA U. (2009). Carnivory is positively correlated with latitude among omnivorous mammals: evidence from brown bears, badgers and pine martens. Annales Zoologici Fennici 46: 395-415.

Wade D.A. \& Bowns J.E. (1985). Procedures for Evaluating Predation on Livestock and Wildlife. B-1429. Texas Agricultural Extension Service, Texas Agricultural Experiment Station, and United State Fish \& Wildlife Service, San Angelo, Texas. 
WANG H. \& Fuller T.K. (2003). Food habits of four sympatric carnivores in southeastern China. Mammalia 67: 513-519. https://doi.org/10.1515/mamm-2003-0405

WeBER D. (1989). The diet of polecats (Mustela putorius L.) in Switzerland. Zeitschrift für Säugetierkunde 54: $157-171$.

Wegge P., Shrestha R. \& Flagstad Ø. (2012). Snow leopard Panthera uncia predation on livestock and wild prey in a mountain valley in northern Nepal: implications for conservation management. Wildlife Biology 18: 131-141. https://doi.org/10.2981/11-049

Wei F., Wang Z., Feng Z., Li M. \& Zhou A. (2000). Seasonal energy utilization in bamboo by the red panda (Ailurus fulgens). Zoo Biology 19: 27-33.

https://doi.org/10.1002/(SICI)1098-2361(2000)19:1<27::AID-ZOO3>3.0.CO;2-9

Weigend M., Mustafa A. \& Ensikat H.J. (2018). Calcium phosphate in plant trichomes: the overlooked biomineral. Planta 247: 277-285. https://doi.org/10.1007/s00425-017-2826-1

Wilcomb M.J. (1948). Fox Populations and Food Habits in Relation to Game Birds in the Willamette Valley, Oregon. M.S. Thesis. Oregon State College, Corvallis, Oregon.

WiLSON M.J. (2003). Clay mineralogical and related characteristics of geophagic materials. Journal of Chemical Ecology 29: 1525-1547. https://doi.org/10.1023/A:1024262411676

Wilson V. \& Dookia S. (2019). Desert fox (Vulpes vulpes pusilla) a generalist or opportunistic specialist: Insight into the feeding habit in Thar Desert of Rajasthan, India. Environment and Ecology 37: 1133-1139.

WINK M. (2013). Evolution of secondary metabolites in legumes (Fabaceae). South African Journal of Botany 89: 164-175. https://doi.org/10.1016/j.sajb.2013.06.006

Wise M.H., LinN I.J. \& KenNedy C.R. (1981). A comparison of the feeding biology of mink Mustela vison and otter Lutra lutra. Journal of Zoology 195: 181-213.

https://doi.org/10.1111/j.1469-7998.1981.tb03458.x

Wratten S.D., EdWARDS P.J. \& DunN I. (1984). Wound-induced changes in the palatability of Betula pubescens and B. pendula. Oecologia 61: 372-375. https://doi.org/10.1007/BF00379637

Wolff F. (2001). Vertebrate Ecology in Caatinga: A. Distribution of Wildlife in Relation to Water. B. Diet of Pumas (Puma concolor) and Relative Abundance of Felids. Ph.D. dissertation. University of Missouri-St. Louis, St. Louis, Missouri.

Wood J.E. (1954). Food habits of furbearers of the upland post oak region in Texas. Journal of Mammalogy 35: 406-415. https://doi.org/10.2307/1375965

Wrangham R.W. \& Nishida T. (1983). Aspilia spp. leaves: a puzzle in the feeding behavior of wild chimpanzees. Primates 24: 276-282. https://doi.org/10.1007/BF02381090

Xiong M., Shao X., Long Y., Bu H., Zhang D., Wang D., Li S., Wang R. \& Yao M. (2016). Molecular analysis of vertebrates and plants in scats of leopard cats (Prionailurus bengalensis) in southwest China. Journal of Mammalogy 97: 1054-1064. https://doi.org/10.1093/jmammal/gyw061

XIRouchakis S.M. (2005). The diet of Eurasian griffons (Gyps fulvus) in Crete. Journal of Raptor Research 39: 179-183.

YeAGER L.E. \& ElDER W.H. (1945). Pre- and post-hunting season foods of racoons on an Illinois goose refuge. The Journal of Wildife Management 9: 48-56. https://doi.org/10.2307/3795946 
YiP S.J., Rich M.A. \& Dickman C.R. (2015). Diet of the feral cat, Felis catus, in central Australian grassland habitats during population cycles of its principal prey. Mammal Research 60: 39-50. https://doi.org/10.1007/s13364-014-0208-7

Zanzani S.A., Gazzonis A.L., Scarpa P., Berrilli F. \& Manfredi M.T. (2014). Intestinal parasites of owned dogs and cats from metropolitan and micropolitan areas: prevalence, zoonotic risks, and pet owner awareness in northern Italy. BioMed Research International 2014: 696508.

https://doi.org/10.1155/2014/696508

Zapata S.C., Travaini A. \& Martínez-Peck R. (2001). Seasonal feeding habits of the Patagonian hognosed skunk Conepatus humboldtii in southern Patagonia. Acta Theriologica 46: 97-102.

https://doi.org/10.1007/BF03192421

Zhiryakov V.A. \& BAidAVLetov R.Z. (2002). Ecology and behaviour of the snow leopard in Kazakhstan. Selevinia 2002: 1-4.

Zhou Y., WANG S.R. \& MA J.Z. (2017). Comprehensive species set revealing the phylogeny and biogeography of Feliformia (Mammalia, Carnivora) based on mitochondrial DNA. PloS ONE 12: e0174902. https://doi.org/10.1371/journal.pone.0174902

Zhou Y., Chen W., Kaneko Y., Newman C., Liao Z., Zhu X., Buesching C.D., Xie Z. \& MACDONALD D.W. (2015). Seasonal dietary shifts and food resource exploitation by the hog badger (Arctonyx collaris) in a Chinese subtropical forest. European Journal of Wildlife Research 61: 125-133. https://doi.org/10.1007/s10344-014-0881-5

Zielinski W.J., Duncan N.P., Farmer E.C., Truex R.L., Clevenger A.P. \& Barrett R.H. (1999). Diet of fishers (Martes pennanti) at the southernmost extent of their range. Journal of Mammalogy 80: 961-971. https://doi.org/10.2307/1383266

Zumpt I.F. (1968). The feeding habits of the yellow mongoose, Cynictis penicillata, the suricate, Suricata suricatta and the cape ground squirrel, Xerus inauris. Journal of the South African Veterinary Medical Association 39: 89-91.

Žunna A., Ozolinsš J. \& Pupila A. (2009). Food habits of the wolf Canis lupus in Latvia based on stomach analyses. Estonian Journal of Ecology 58: 141-152.

Manuscript received: 15 August 2019

Manuscript accepted: 6 April 2020

Published on: 2 July 2020

Branch editor: Ellen Decaesstecker 


\section{Appendix 1}

Hundreds of hours of observations of Kait and Alan's dog (Canis familiaris) living in Florida, USA over the course of six years reveal that the dog frequently consumes a few pieces of unmowed grass blades (typically several times per week). This dog has consumed several different genera of Poaceae, including Cortaderia, Dactyloctenium, Eleusine, Neyraudia, Sorghum, and Urochloa. One species of Cyperaceae (Cyperus croceus) was also consumed. During a visit to a friend in Seattle, Washington, USA, his two dogs were observed eating unmowed patches of Festuca rubra adjacent to the mowed lawn, said to be a frequent behavior by the owner. In all instances with the above three dogs, it was noticed that the dog preferred to consume long, uncut grass blades that were near head height, and the dogs avoided cut, mown short grass. Vomiting or nausea was not observed in any of these instances. Vouchers of the actual plants consumed are deposited in the University of South Florida herbarium (collection nos 4039, 4063, 4064, 4077, 4082, 4083, 4795, and s.n. [Aug. 2016]). 